\title{
Tubular Perylene bisimide Macrocycles for the Recognition of Geometrical Isomers of Azobenzenes
}

Tankut Türel, Samarth Bhargava, Suresh Valiyaveettil ${ }^{*}$

Department of Chemistry, National University of Singapore, 3 Science Drive 3, 117543, Singapore

*E-mail:chmsv@nus.edu.sg

\section{Supporting Information}

\section{Contents}

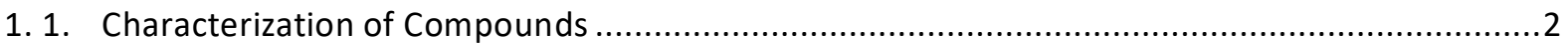

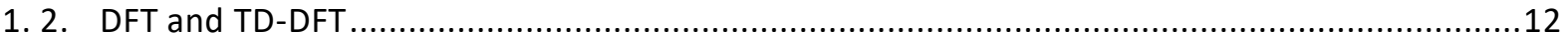

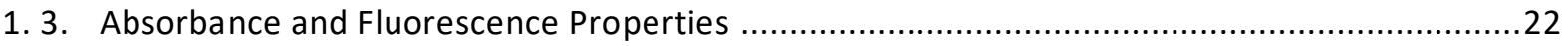

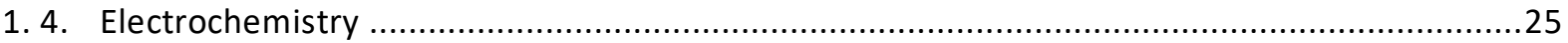

1. 5. UV-Vis and Fluorescence Titrations of Macrocycles with Azobenzene Derivatives .................26

1. 6. 3D Optimized Structures and Frontier Energy Levels of the Titrants and Stabilized complexes

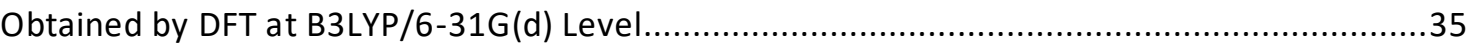

1. 7. ${ }^{1} \mathrm{H}$ NMR Titration of macrocycle $A$ with $Z$ and $E$-azobenzene.....................................40

1. 8. ${ }^{1} \mathrm{H}-{ }^{1} \mathrm{H}$ NOESY NMR of macrocycle $\mathrm{A}$ in the presence of $\mathrm{E}$-4-dimethylaminoazobenzene .........42

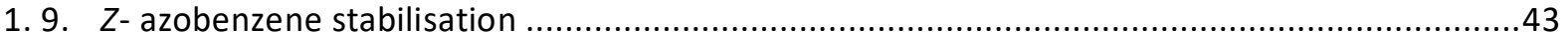

1. 10. UV-Vis and fluorescence titrations of macrocycles with aromatic amines ..........................44

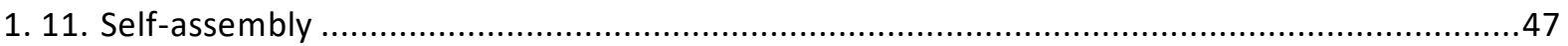


1. 1. Characterization of Compounds

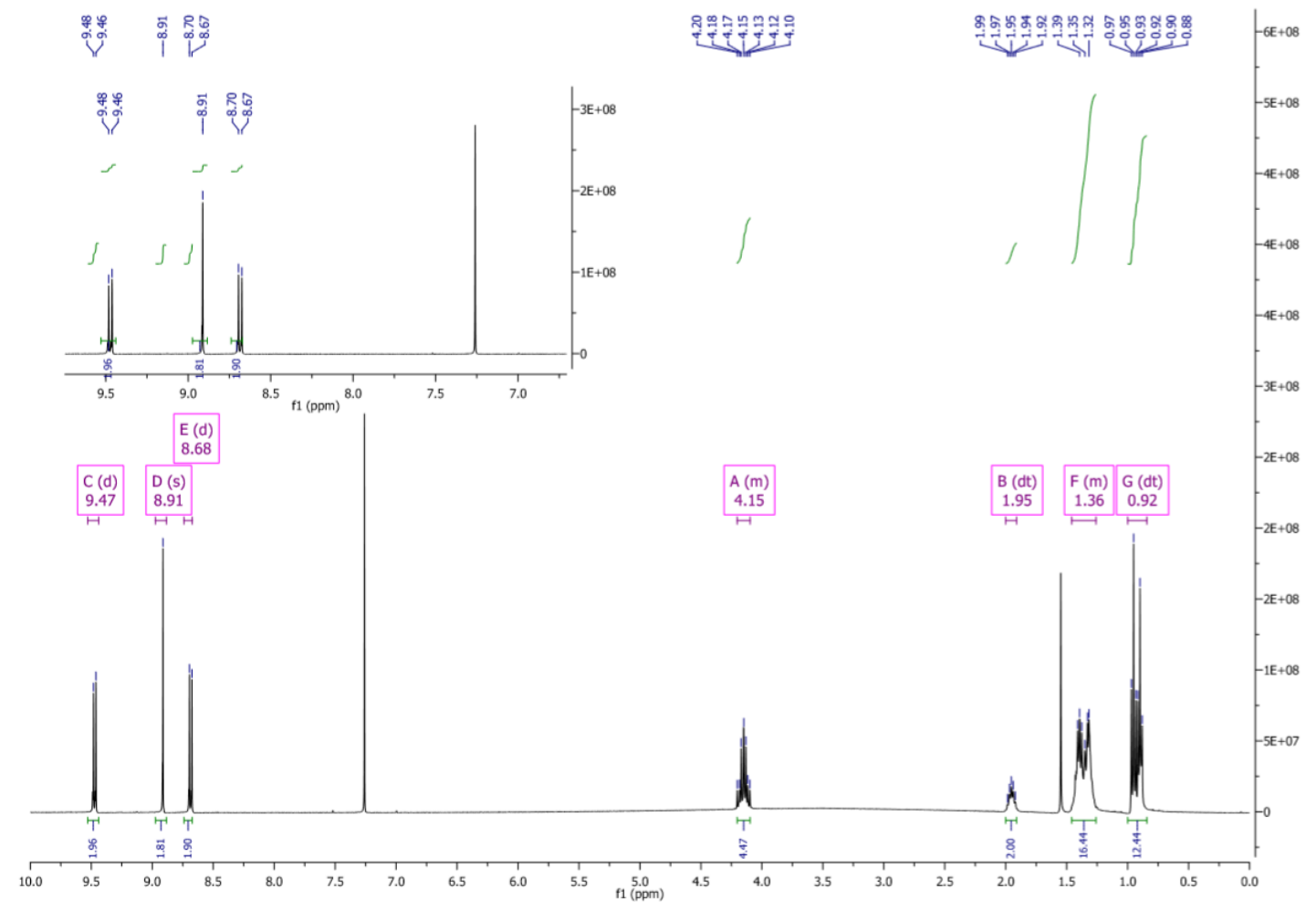

Figure $\mathrm{S} 1 .{ }^{1} \mathrm{H}-\mathrm{NMR}$ spectra of $\mathrm{PBIBr}_{2}$ in $\mathrm{CDCl}_{3}$ 


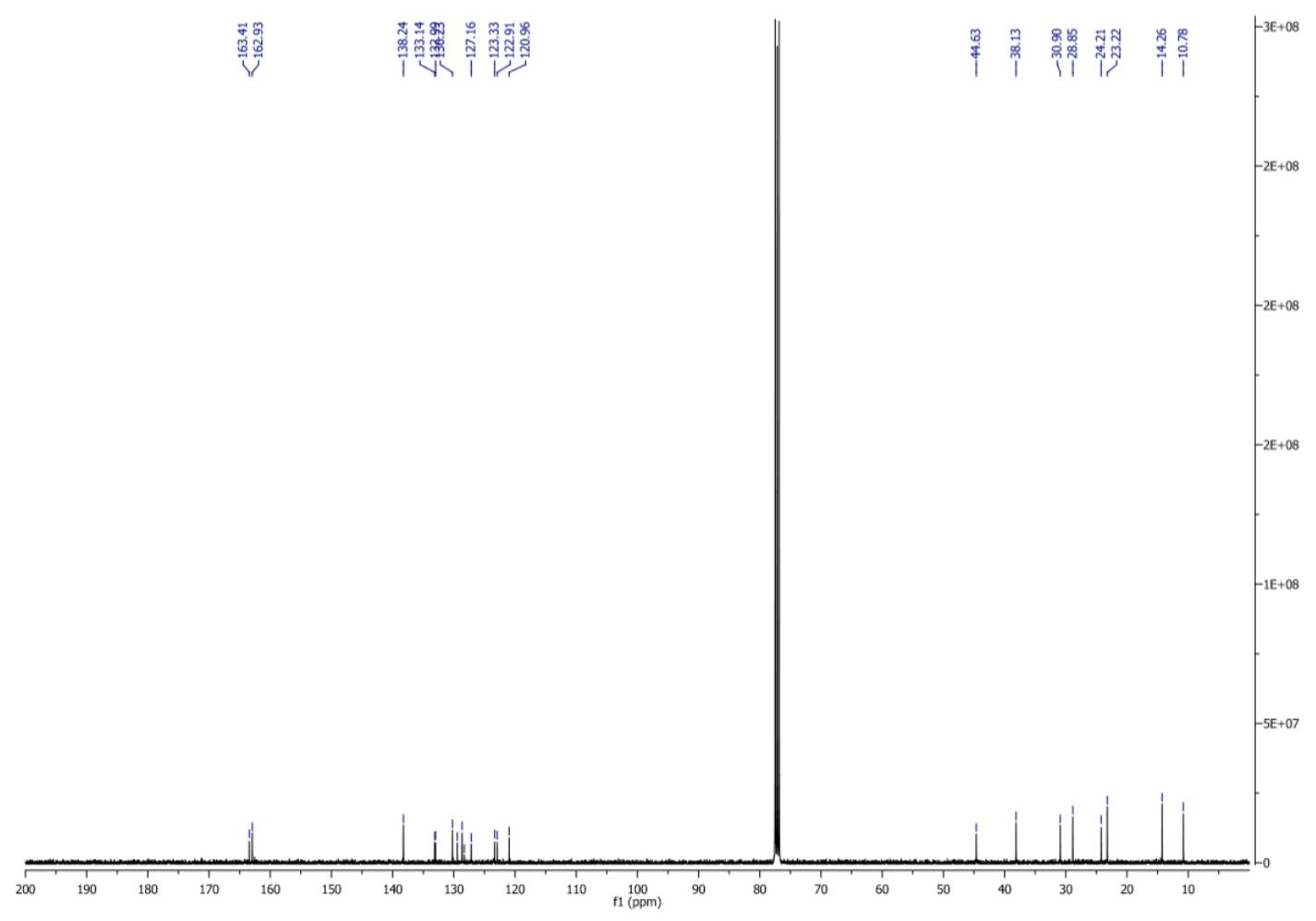

Figure $\mathrm{S} 2 .{ }^{13} \mathrm{C}\left\{{ }^{1} \mathrm{H}\right\}-\mathrm{NMR}$ spectra of $\mathrm{PBIBr}_{2}$ in $\mathrm{CDCl}_{3}$

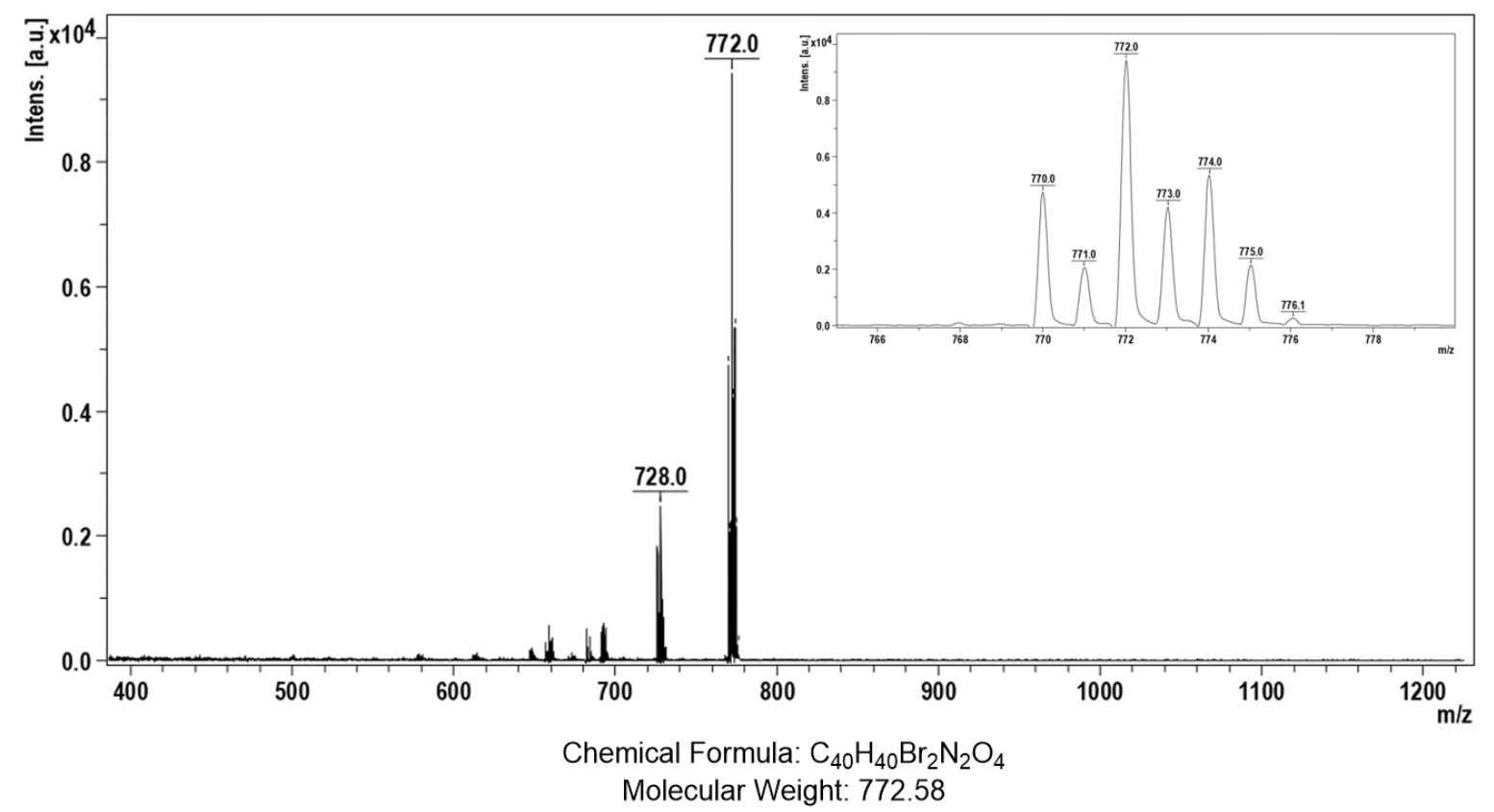

m/z: 772.13 (100.0\%), 770.14 (51.4\%), 774.13 (48.6\%), 773.14 (43.3\%), $775.13(21.0 \%), 771.14$ (16.7\%), 774.14 (6.5\%), 771.14 (5.6\%), $772.14(4.7 \%), 776.14$ (4.4\%), $774.14(2.6 \%)$

Figure S3. MALDI-TOF spectra of $\mathrm{PBIBr}_{2}$ 


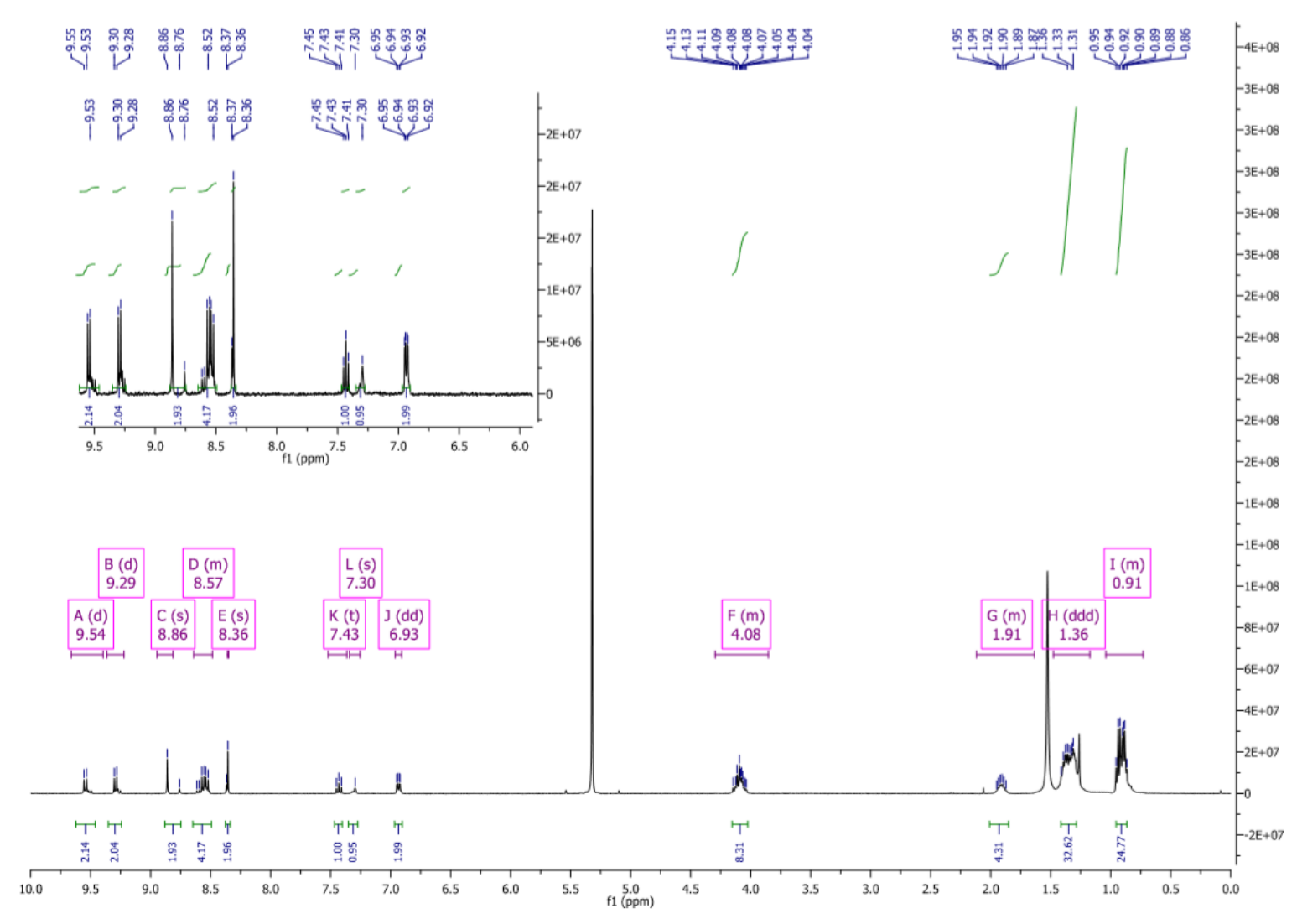

Figure S4. ${ }^{1} \mathrm{H}-\mathrm{NMR}$ spectra of $\mathrm{PBI}_{2} \mathrm{RBr}_{2}$ in $\mathrm{CD}_{2} \mathrm{Cl}_{2}$

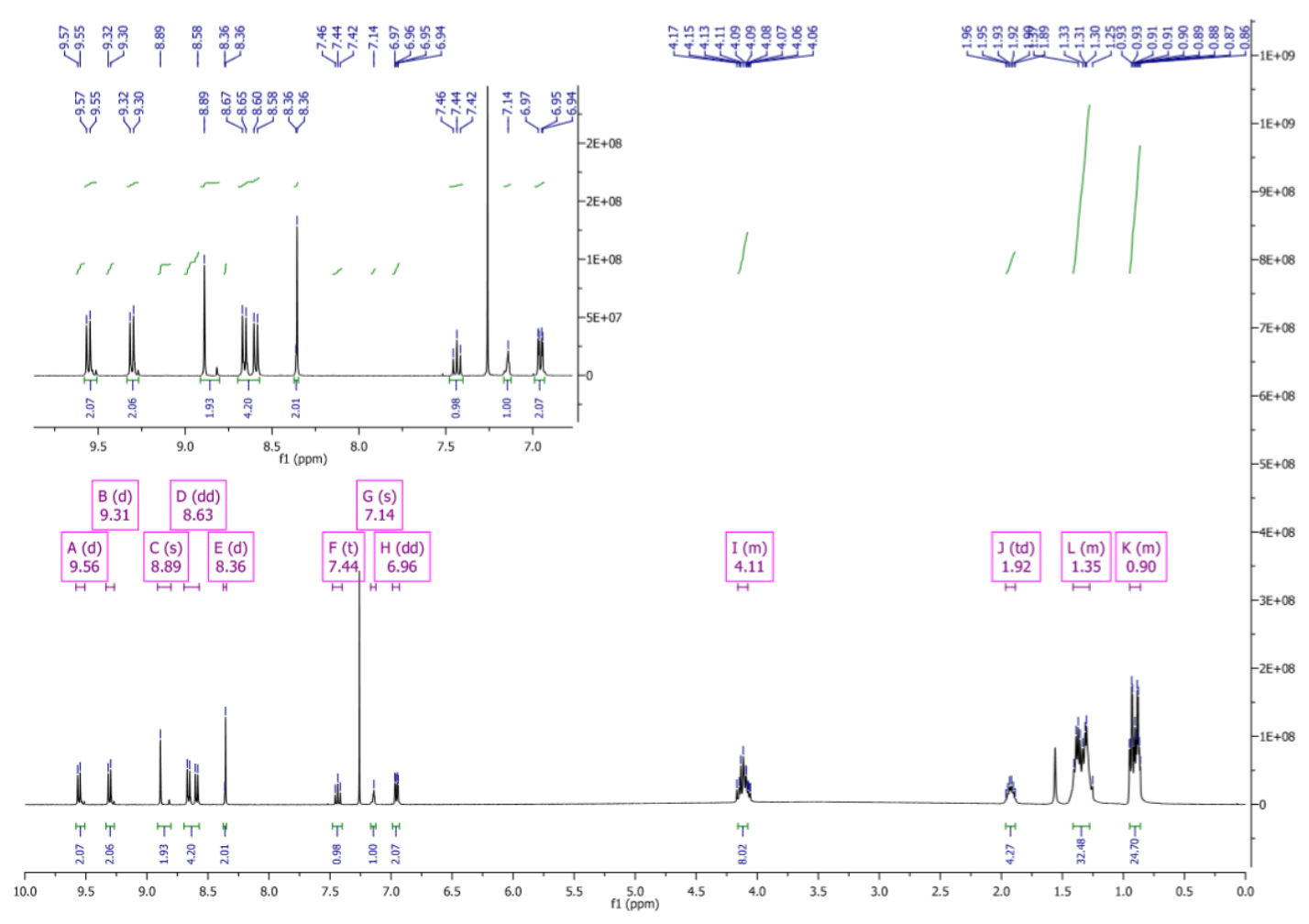

Figure S5. ${ }^{1} \mathrm{H}-\mathrm{NMR}$ spectra of $\mathrm{PBI}_{2} \mathrm{RBr}_{2}$ in $\mathrm{CDCl}_{3}$ 


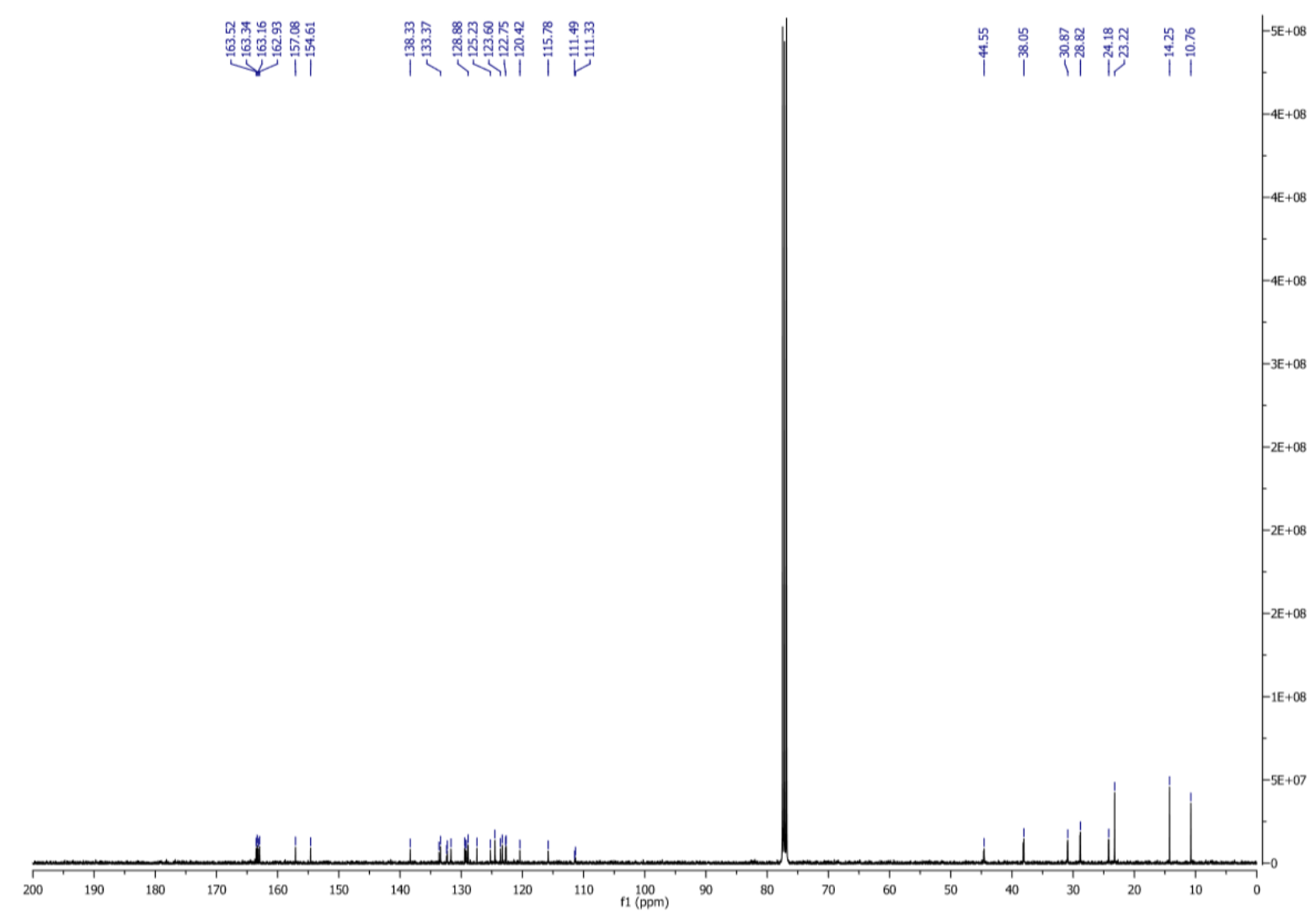

Figure S6. ${ }^{13} \mathrm{C}\left\{{ }^{1} \mathrm{H}\right\}-\mathrm{NMR}$ spectra of $\mathrm{PBI}_{2} \mathrm{RBr}_{2}$ in $\mathrm{CDCl}_{3}$ 


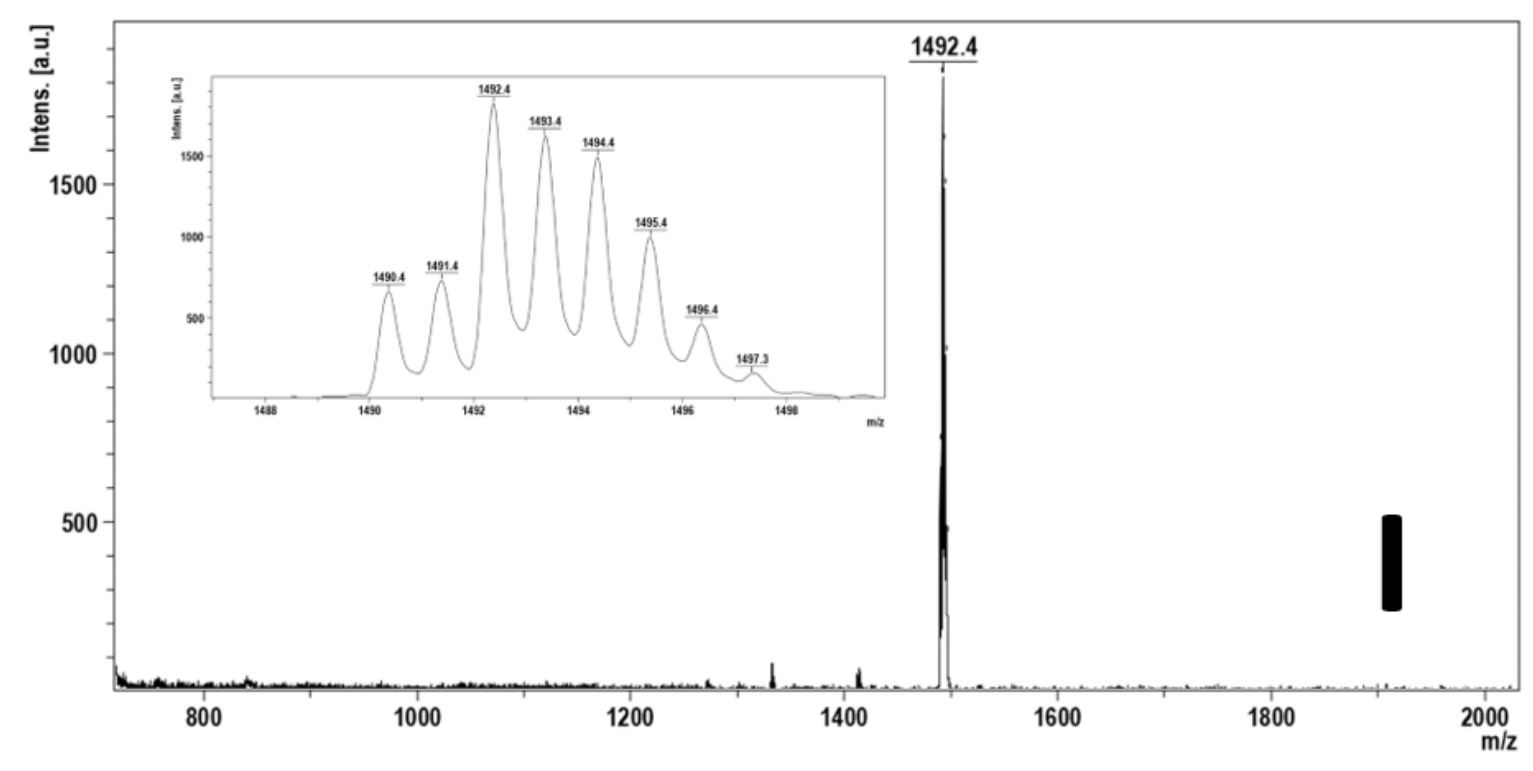

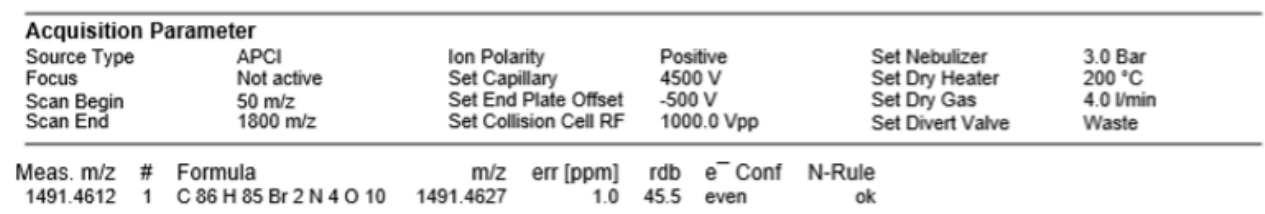

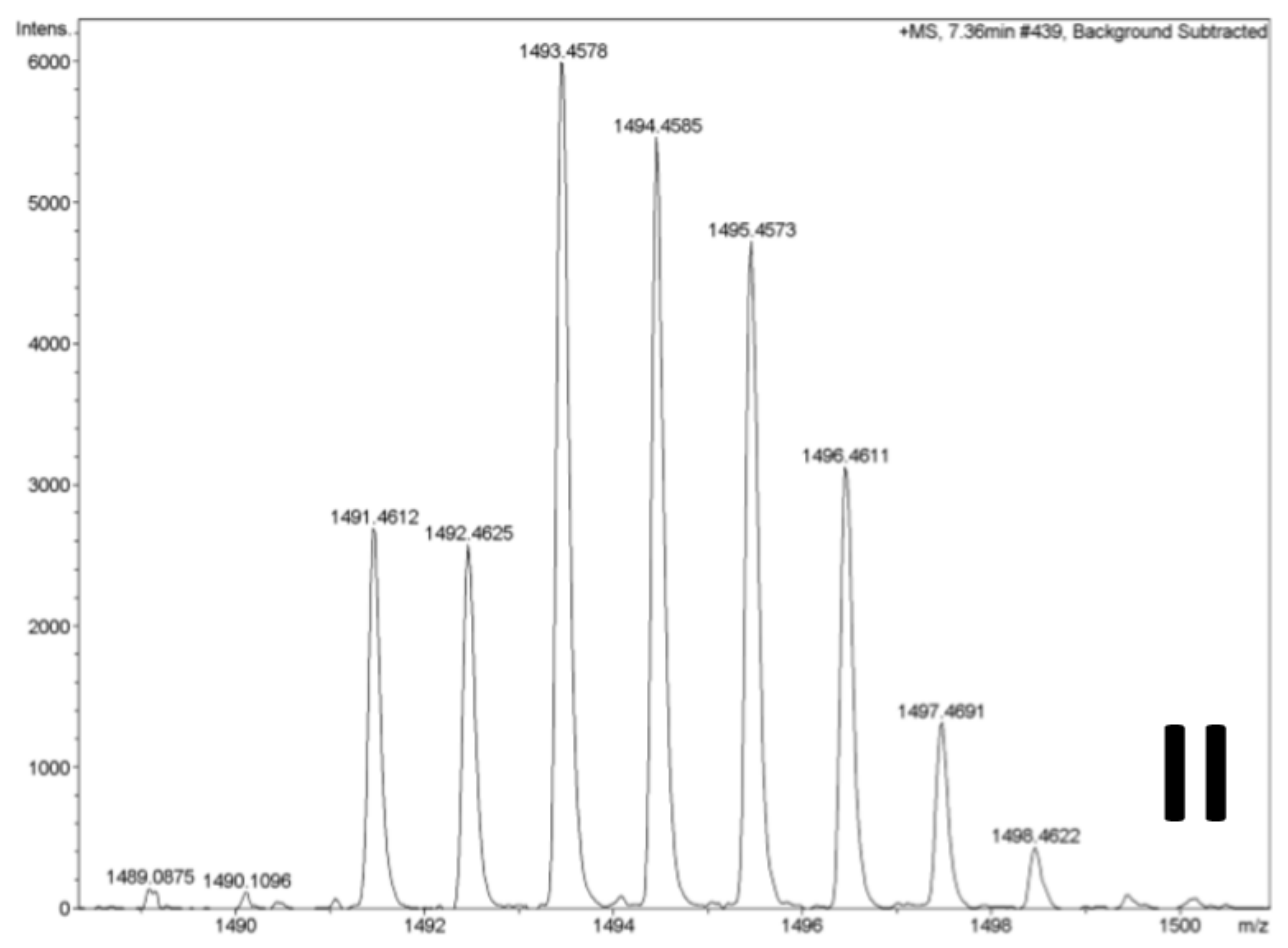

Figure S7. MALDI-TOF spectra in negative ion polarity (I) and HRMS (APCI-TOF) spectra in positive ion polarity (II). 


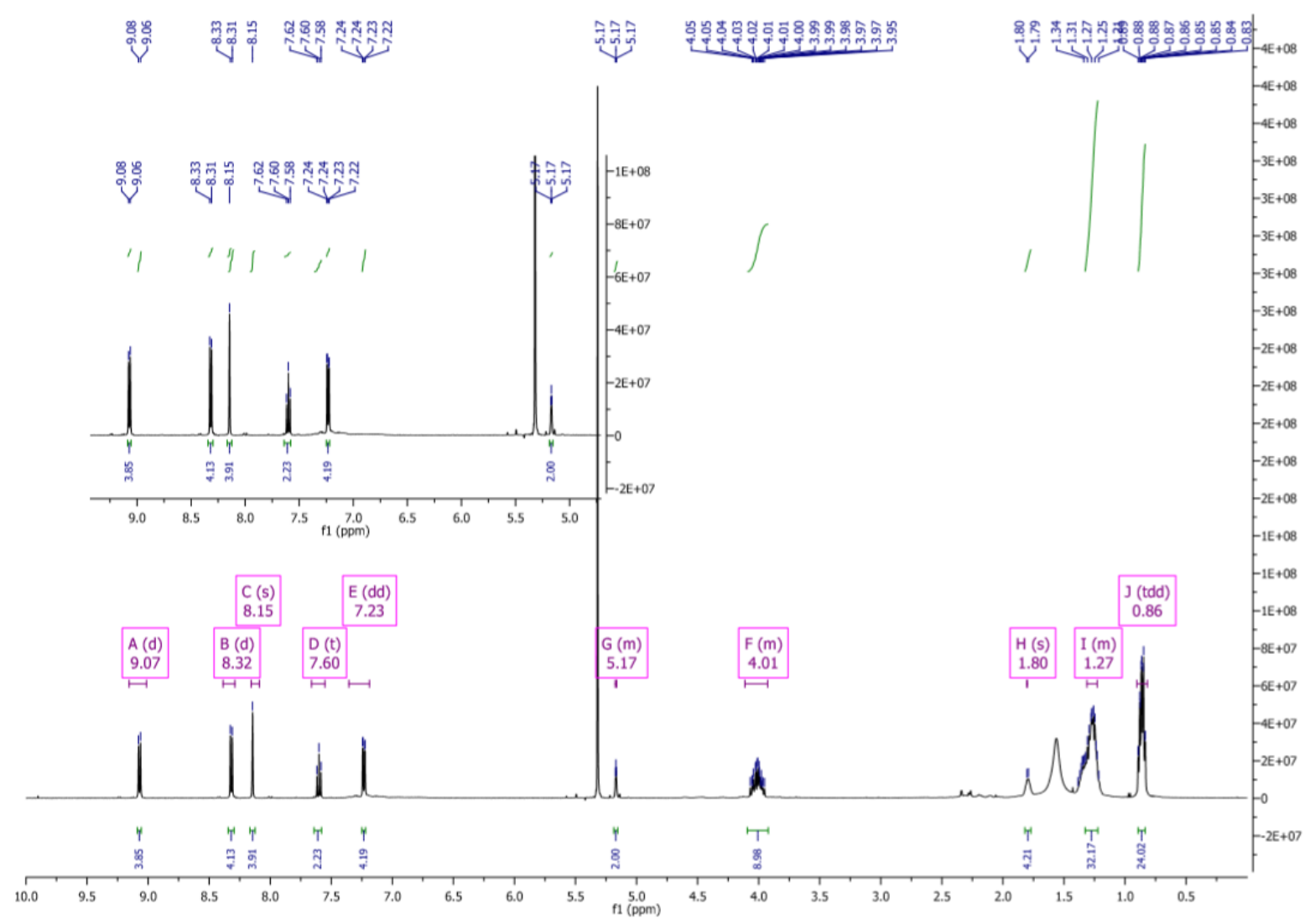

Figure S8. ${ }^{1} \mathrm{H}-\mathrm{NMR}$ spectra of macrocycle $\mathrm{A}$ in $\mathrm{CD}_{2} \mathrm{Cl}_{2}$

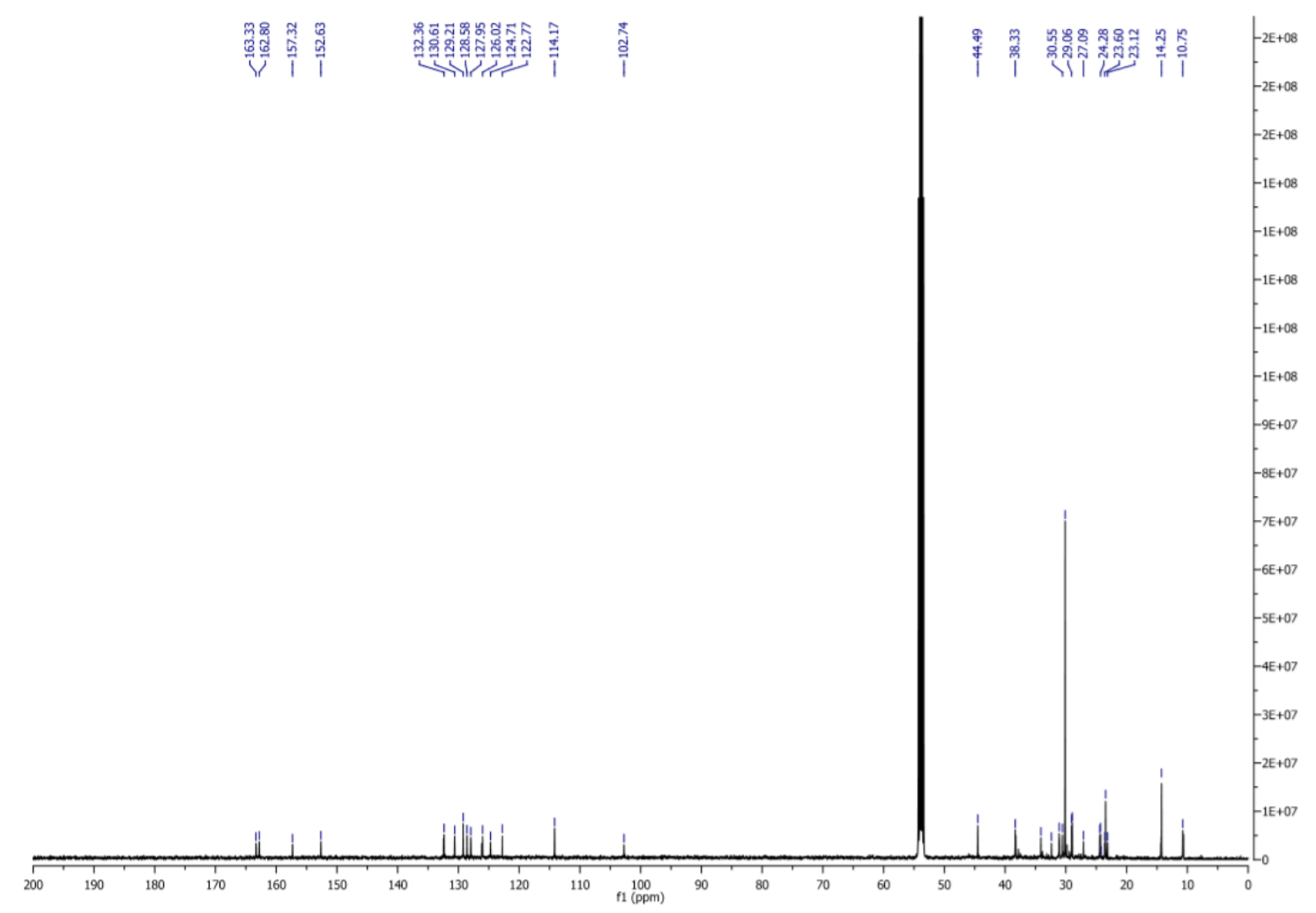

Figure S9. ${ }^{13} \mathrm{C}\left\{{ }^{1} \mathrm{H}\right\}$-NMR spectra of macrocycle $\mathrm{A}$ in $\mathrm{CD}_{2} \mathrm{Cl}_{2}$ 


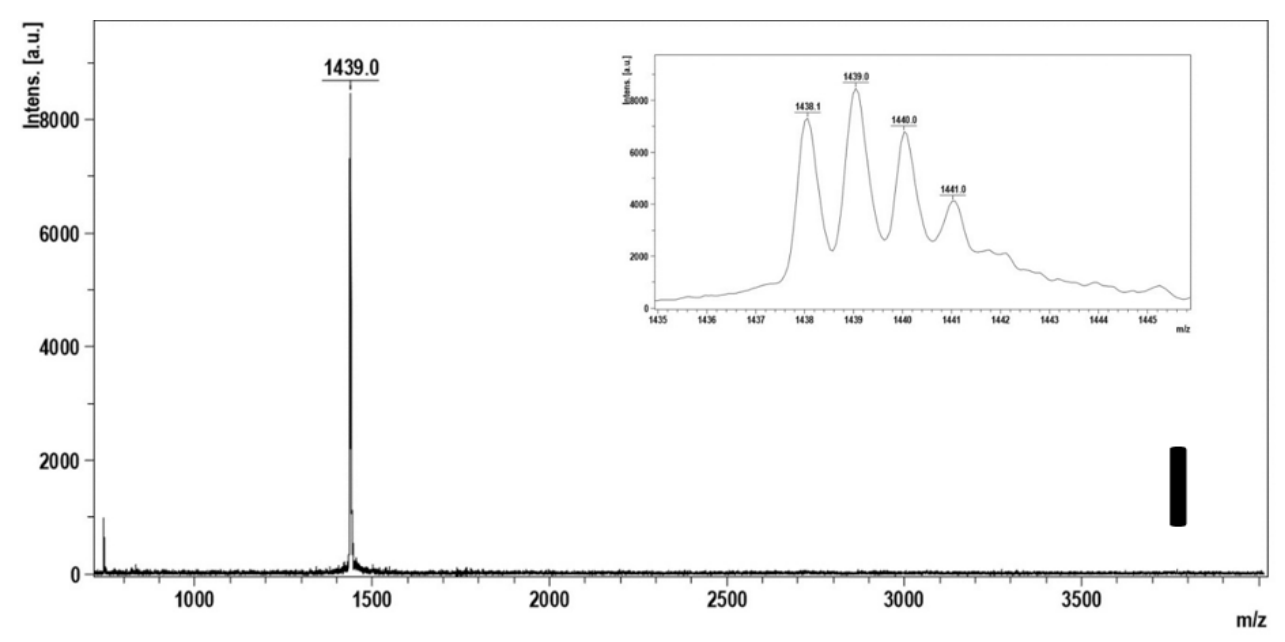

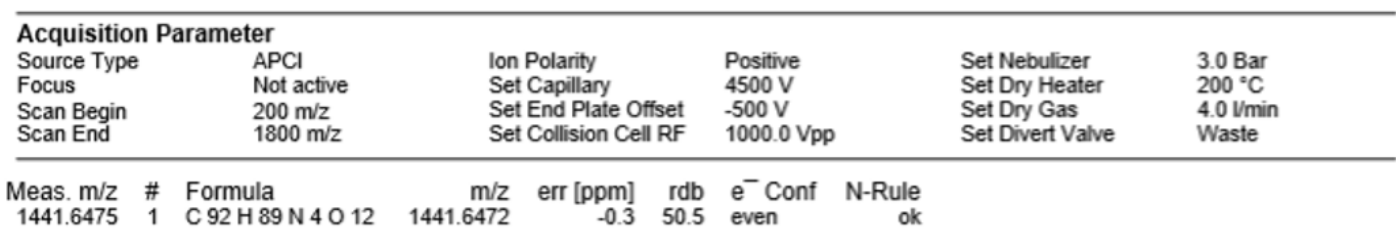

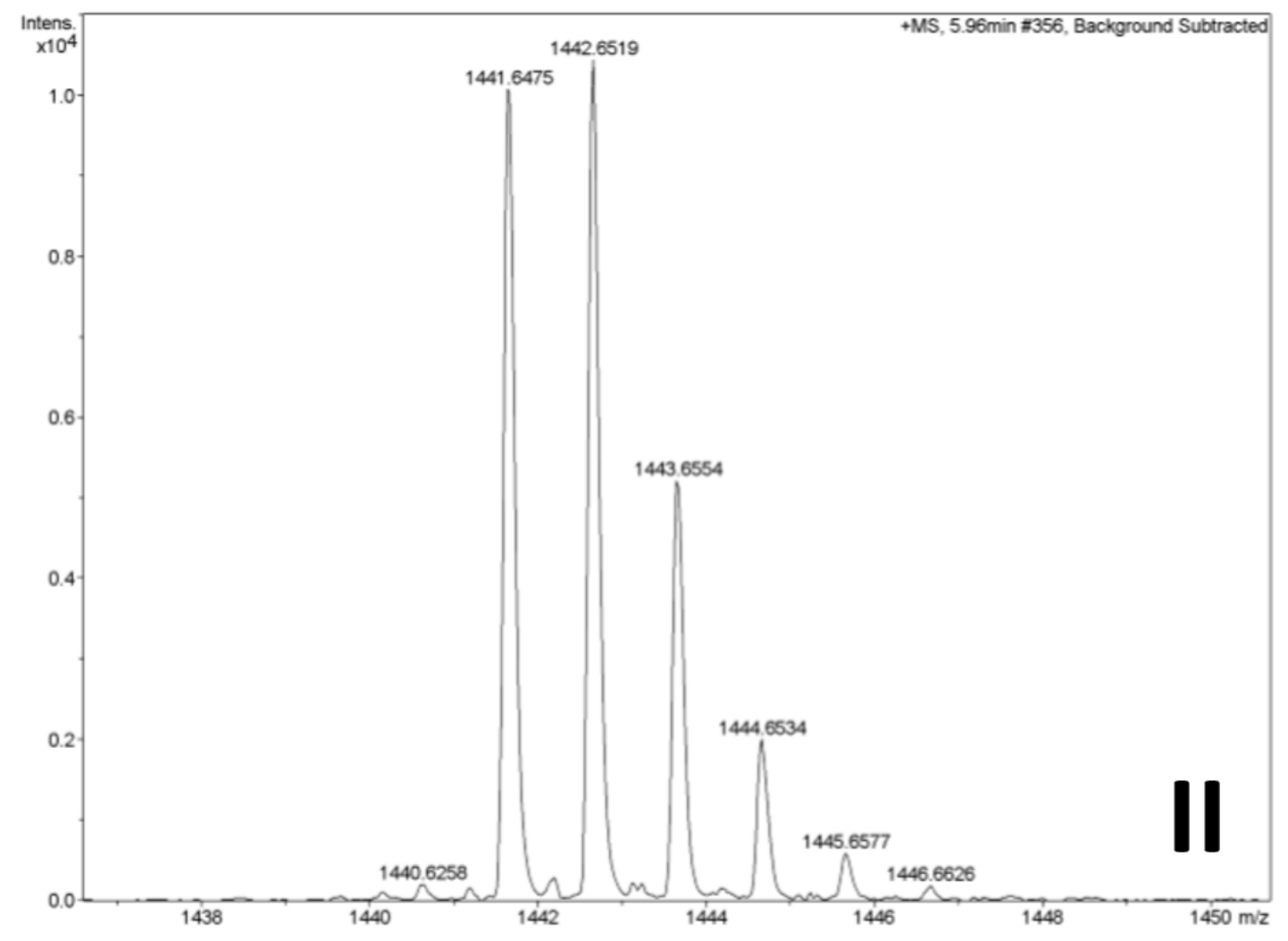

Figure S10. MALDI-TOF spectra in negative ion polarity (I) and HRMS (APCI-TOF) spectra in positive ion polarity (II) 


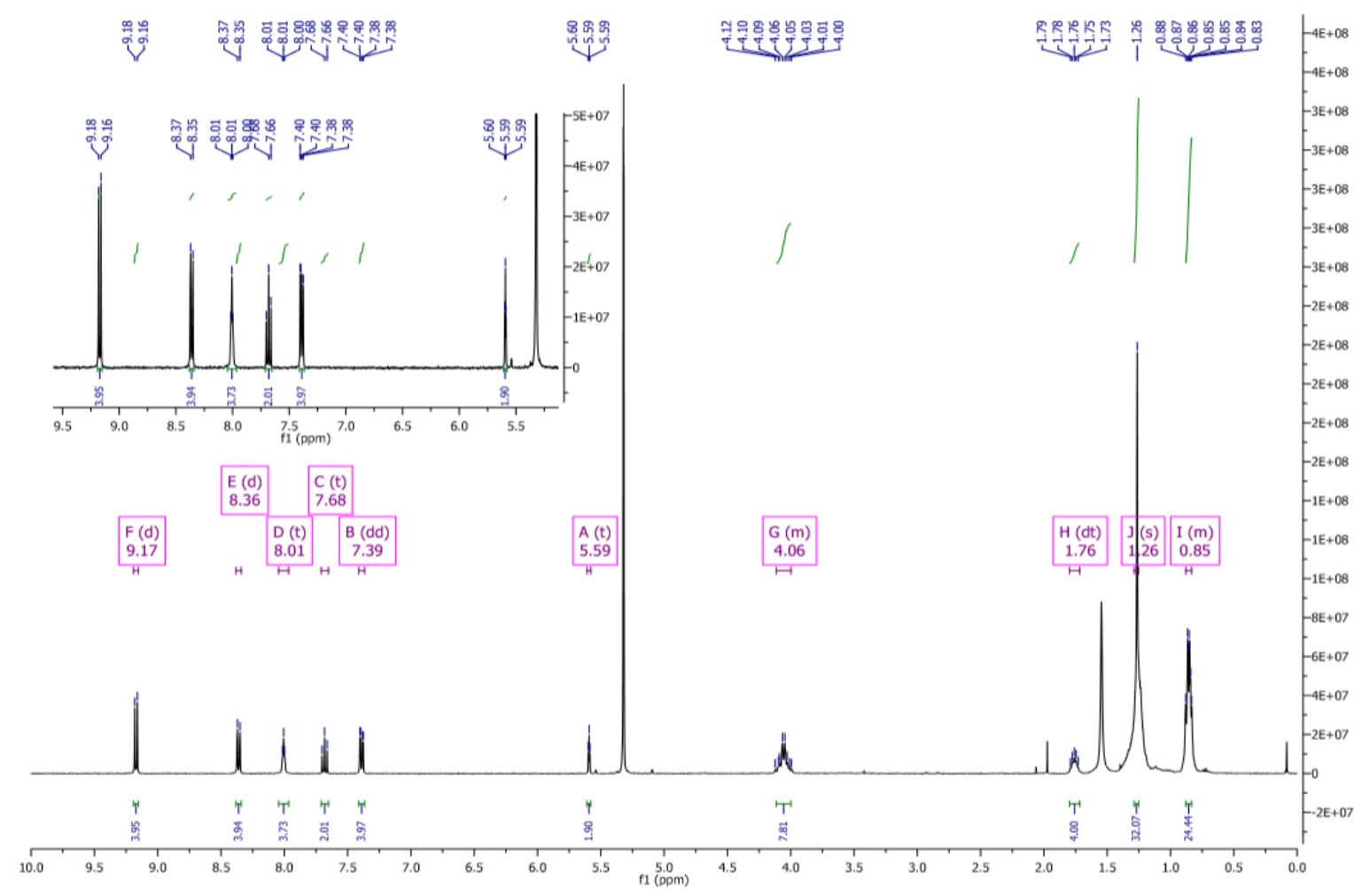

Figure S11. ${ }^{1} \mathrm{H}-\mathrm{NMR}$ spectra of macrocycle $\mathrm{B}$ in $\mathrm{CD}_{2} \mathrm{Cl}_{2}$

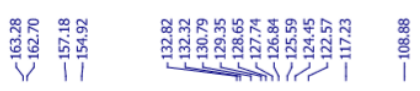

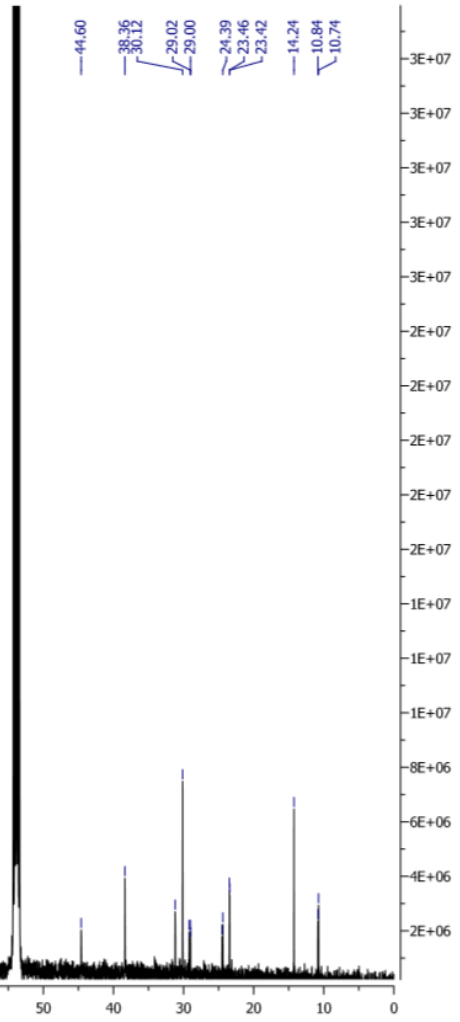

Figure S12. ${ }^{13} \mathrm{C}\left\{{ }^{1} \mathrm{H}\right\}-\mathrm{NMR}$ Spectra of macrocycle $\mathrm{B}$ in $\mathrm{CD}_{2} \mathrm{Cl}_{2}$ 


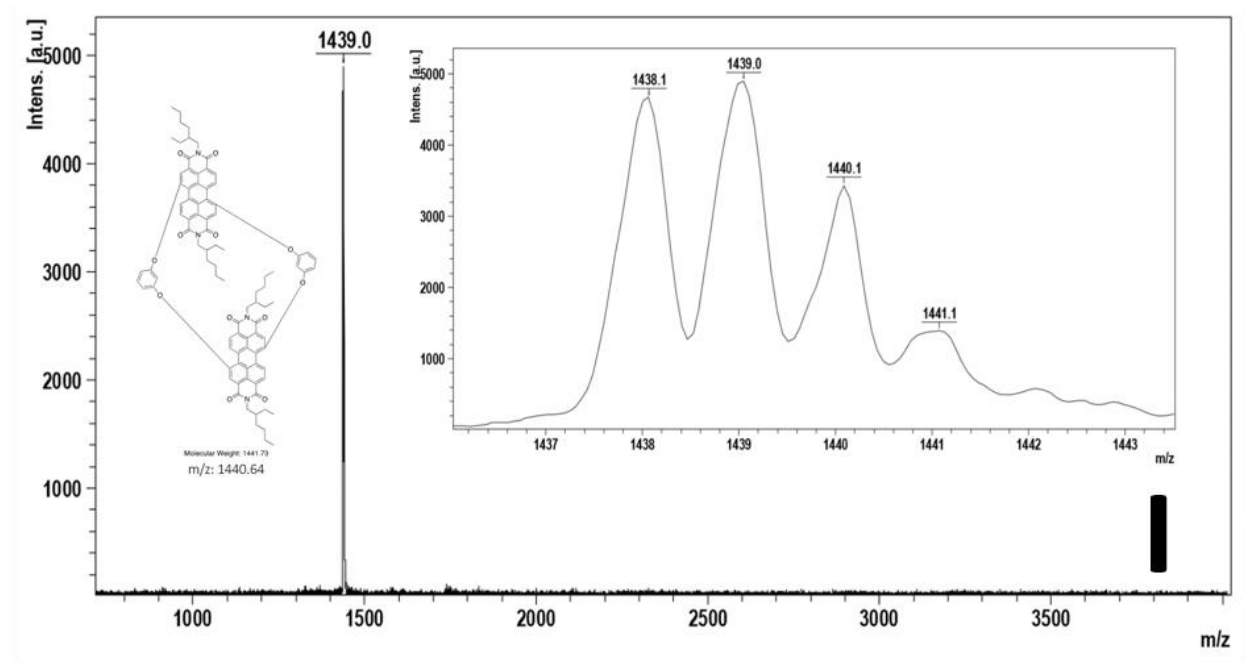

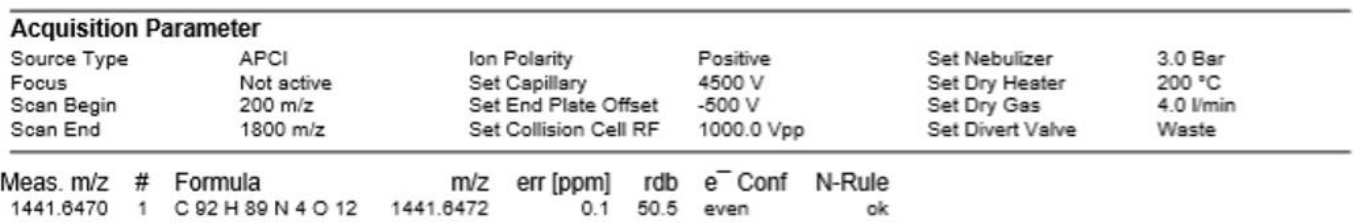

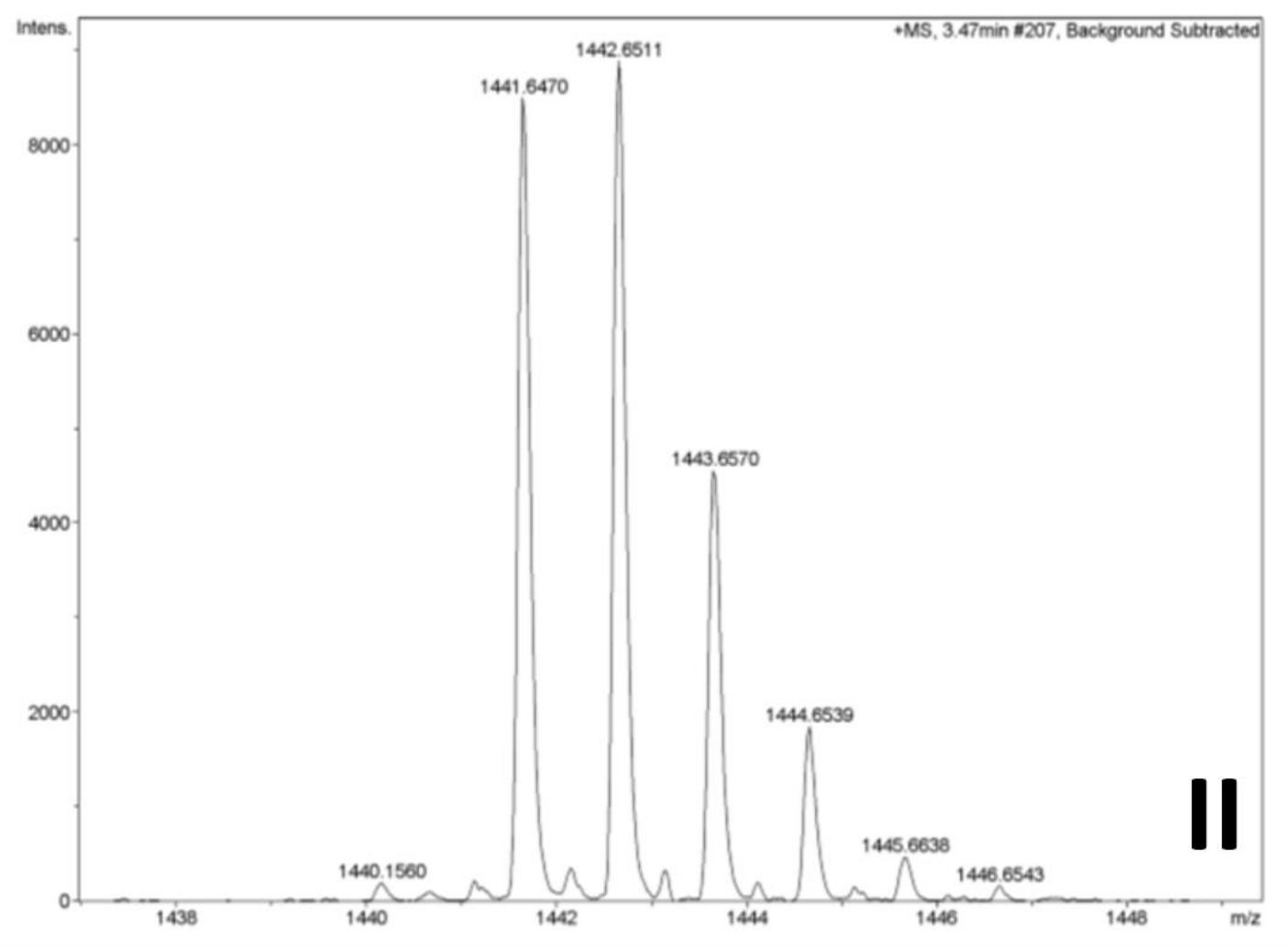

Figure S13. MALDI-TOF spectra of macrocycle B in negative ion polarity (I) and HRMS (APCI-

TOF) spectra in positive ion polarity (II). 


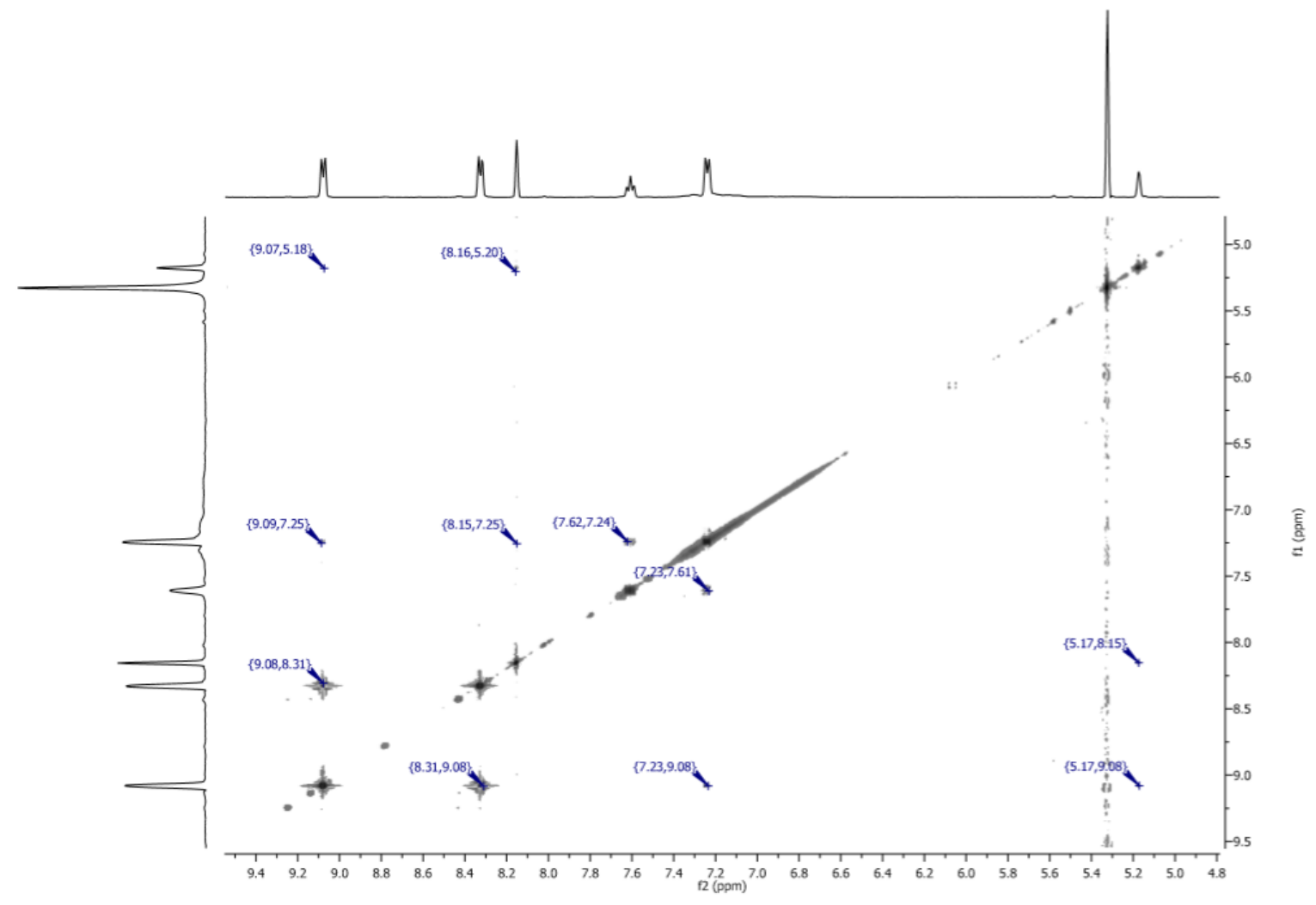

Figure S14. ${ }^{1} \mathrm{H}-{ }^{1} \mathrm{H}$ NOESY spectra of macrocycle $\mathrm{A}$ in $\mathrm{CD}_{2} \mathrm{Cl}_{2}$.

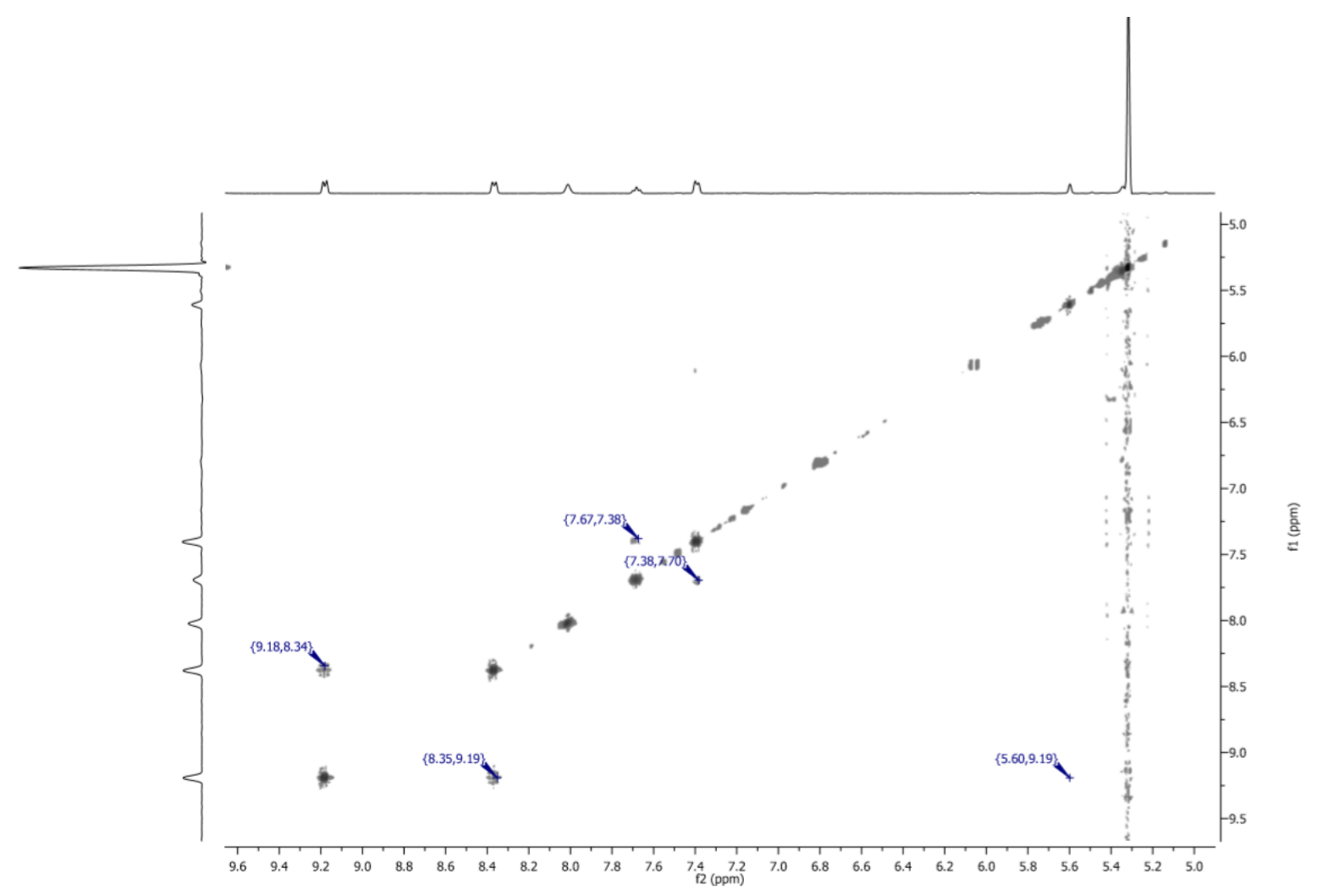

Figure S15. ${ }^{1} \mathrm{H}-{ }^{1} \mathrm{H}$ NOESY spectra of macrocycle $\mathrm{B}$ in $\mathrm{CD}_{2} \mathrm{Cl}_{2}$. 


\section{2. DFT and TD-DFT}

Table S1. Summarized DFT data of macrocycles (C1, C2, C3 and C4 are central middle carbons of perylene backbone).

\begin{tabular}{lcc}
\hline \multicolumn{1}{c}{ DFT DATA } & Macrocycle A & Macrocycle B \\
\hline Imide N1-N1' Distance & $6.06 \AA$ & $6.67 \AA$ \\
C1-C1' Distance & $5.92 \AA$ & $5.99 \AA$ \\
C2-C2' Distance & $5.86 \AA$ & $5.92 \AA$ \\
C3-C3' Distance & $5.86 \AA$ & $6.00 \AA$ \\
C4-C4' Distance & $5.92 \AA$ & $6.16 \AA$ \\
Imide N2-N2' Distance & $6.06 \AA$ & $6.34 \AA$ \\
Resorcinol Moiety Diagonal & $9.02 \AA$ & $9.04 \AA$ \\
Oxygen O1-O3 Distance & & \\
Resorcinol Moiety Diagonal & $9.00 \AA$ & $8.92 \AA$ \\
Oxygen O1-O3 Distance & & \\
Dihedral Angle (1) & $13.9^{\circ}$ & $14.2^{\circ}$ \\
Dihedral Angle (2) & $13.9^{\circ}$ & $14.5^{\circ}$ \\
Dihedral Angle (3) & $13.8^{\circ}$ & $14.3^{\circ}$ \\
Dihedral Angle (4) & $13.9^{\circ}$ & $14.5^{\circ}$ \\
HOMO (eV) & -6.22 & -6.16 \\
LUMO (eV) & -3.70 & -3.67 \\
Band Gap (eV) & 2.52 & 2.49 \\
\hline
\end{tabular}

Table S2. Symbolic Z-matrix of macrocycle A, Charge $=0$, Multiplicity $=1$.

\begin{tabular}{|l|r|r|r|}
\hline C & 0.9991 & -1.45901 & 4.81668 \\
\hline C & 2.01382 & -0.75823 & 4.1531 \\
\hline C & 2.94711 & -1.45474 & 3.36427 \\
\hline C & 2.8326 & -2.84307 & 3.20874 \\
\hline C & 1.79488 & -3.53629 & 3.84053 \\
\hline C & 0.89399 & -2.84837 & 4.66593 \\
\hline C & 4.00037 & -0.76533 & 2.73926 \\
\hline C & 3.7693 & -3.54137 & 2.44025 \\
\hline H & 0.12224 & -3.38426 & 5.17801 \\
\hline C & 4.85436 & -2.86265 & 1.86832 \\
\hline C & 4.98536 & -1.47653 & 2.0278 \\
\hline H & 5.58607 & -3.40572 & 1.30726 \\
\hline C & 1.29285 & 2.89245 & 5.15524 \\
\hline C & 2.24125 & 3.58045 & 4.39231 \\
\hline C & 3.16907 & 2.87044 & 3.63055 \\
\hline C & 3.11315 & 1.46956 & 3.58209 \\
\hline C & 2.11247 & 0.77374 & 4.29036 \\
\hline C & 1.23042 & 1.49185 & 5.12686 \\
\hline
\end{tabular}




\begin{tabular}{|c|c|c|c|}
\hline $\mathrm{H}$ & 0.60762 & 3.44051 & 5.76772 \\
\hline $\mathrm{C}$ & 4.1699 & 3.56055 & 2.93705 \\
\hline$C$ & 4.07071 & 0.76531 & 2.83585 \\
\hline$C$ & 5.09654 & 1.45415 & 2.17725 \\
\hline $\mathrm{C}$ & 5.14919 & 2.85093 & 2.23212 \\
\hline $\mathrm{H}$ & 5.93525 & 3.37702 & 1.73201 \\
\hline C & 4.20935 & 5.09958 & 2.94933 \\
\hline$C$ & 1.63877 & -5.05576 & 3.64147 \\
\hline 0 & 0.84799 & -5.6978 & 4.38031 \\
\hline 0 & 5.22734 & 5.69526 & 2.51072 \\
\hline C & 3.62514 & -5.05934 & 2.22541 \\
\hline$C$ & 2.2599 & 5.12029 & 4.41261 \\
\hline 0 & 1.5605 & 5.73536 & 5.25886 \\
\hline 0 & 4.57868 & -5.70402 & 1.71676 \\
\hline $\mathrm{N}$ & 3.07077 & 5.88716 & 3.4518 \\
\hline $\mathrm{N}$ & 2.38746 & -5.76917 & 2.59164 \\
\hline $\mathrm{C}$ & 2.7289 & -7.11593 & 3.0722 \\
\hline $\mathrm{H}$ & 3.25485 & -7.64573 & 2.30553 \\
\hline $\mathrm{H}$ & 3.34842 & -7.03791 & 3.94103 \\
\hline $\mathrm{H}$ & 1.83209 & -7.64442 & 3.31978 \\
\hline$C$ & 3.5764 & 7.10138 & 4.1078 \\
\hline $\mathrm{H}$ & 4.19673 & 6.82769 & 4.93562 \\
\hline $\mathrm{H}$ & 4.14825 & 7.6733 & 3.40732 \\
\hline $\mathrm{H}$ & 2.75221 & 7.68695 & 4.45802 \\
\hline $\mathrm{C}$ & -0.50725 & -0.23496 & 5.46604 \\
\hline$C$ & -0.69925 & -1.35298 & 6.29062 \\
\hline $\mathrm{C}$ & -1.09701 & -0.20142 & 4.18525 \\
\hline $\mathrm{C}$ & -1.50867 & -2.41256 & 5.87306 \\
\hline $\mathrm{H}$ & -0.22493 & -1.39329 & 7.24892 \\
\hline $\mathrm{C}$ & -1.95031 & -1.25709 & 3.78717 \\
\hline $\mathrm{H}$ & -0.91105 & 0.62072 & 3.52612 \\
\hline$C$ & -2.14385 & -2.35775 & 4.63096 \\
\hline $\mathrm{H}$ & -1.64421 & -3.26433 & 6.50618 \\
\hline $\mathrm{H}$ & -2.78071 & -3.16033 & 4.32223 \\
\hline$C$ & 5.79324 & -0.18043 & 0.22647 \\
\hline $\mathrm{C}$ & 6.6183 & 0.81578 & -0.31862 \\
\hline $\mathrm{C}$ & 4.62974 & -0.5519 & -0.43563 \\
\hline $\mathrm{C}$ & 6.26379 & 1.43719 & -1.52857 \\
\hline $\mathrm{H}$ & 7.51761 & 1.1013 & 0.18611 \\
\hline$C$ & 4.24612 & 0.10513 & -1.60644 \\
\hline $\mathrm{H}$ & 4.01924 & -1.33673 & -0.04029 \\
\hline $\mathrm{C}$ & 5.07002 & 1.08219 & -2.17514 \\
\hline $\mathrm{H}$ & 6.9023 & 2.18244 & -1.95491 \\
\hline $\mathrm{H}$ & 4.7903 & 1.55723 & -3.09229 \\
\hline $\mathrm{C}$ & -1.91141 & -2.00596 & 1.58015 \\
\hline C & -0.98633 & -1.38512 & 0.74553 \\
\hline
\end{tabular}




\begin{tabular}{|c|c|c|c|}
\hline $\mathrm{C}$ & -2.12144 & -3.38907 & 1.4916 \\
\hline C & -0.82561 & 0.1436 & 0.78751 \\
\hline$C$ & -0.20449 & -2.15543 & -0.12117 \\
\hline$C$ & -1.38655 & -4.1523 & 0.57431 \\
\hline $\mathrm{H}$ & -2.84393 & -3.86185 & 2.12338 \\
\hline C & 0.15675 & 0.75839 & 0.00968 \\
\hline C & -1.65584 & 0.91814 & 1.60819 \\
\hline$C$ & -0.41129 & -3.53762 & -0.21493 \\
\hline$C$ & 0.7929 & -1.54336 & -0.88184 \\
\hline $\mathrm{C}$ & -1.65007 & -5.6645 & 0.45044 \\
\hline C & 1.00371 & -0.02363 & -0.7813 \\
\hline$C$ & 0.30586 & 2.1506 & 0.03763 \\
\hline$C$ & -1.5139 & 2.31236 & 1.62889 \\
\hline C & 0.37421 & -4.30639 & -1.07839 \\
\hline$C$ & 1.60431 & -2.31492 & -1.72397 \\
\hline 0 & -2.36934 & -6.23912 & 1.30861 \\
\hline $\mathrm{N}$ & -1.0862 & -6.44866 & -0.66282 \\
\hline$C$ & 2.06048 & 0.57335 & -1.46113 \\
\hline C & 1.32375 & 2.7582 & -0.70183 \\
\hline$C$ & -0.54475 & 2.93048 & 0.82631 \\
\hline $\mathrm{H}$ & -2.14734 & 2.90526 & 2.255 \\
\hline $\mathrm{C}$ & 1.39829 & -3.69813 & -1.81797 \\
\hline$C$ & 0.12701 & -5.82017 & -1.21557 \\
\hline C & -0.75365 & -7.7978 & -0.18288 \\
\hline$C$ & 2.22454 & 1.96532 & -1.42655 \\
\hline$C$ & 1.46827 & 4.29121 & -0.71446 \\
\hline $\mathrm{C}$ & -0.41678 & 4.46503 & 0.82644 \\
\hline $\mathrm{H}$ & 2.02039 & -4.28944 & -2.45695 \\
\hline 0 & 0.97007 & -6.5316 & -1.82107 \\
\hline $\mathrm{H}$ & -0.01901 & -7.72971 & 0.59211 \\
\hline $\mathrm{H}$ & -1.63509 & -8.26706 & 0.20151 \\
\hline $\mathrm{H}$ & -0.36472 & -8.37863 & -0.99295 \\
\hline $\mathrm{H}$ & 3.03475 & 2.42238 & -1.95516 \\
\hline 0 & 2.50494 & 4.81058 & -1.20313 \\
\hline$N$ & 0.40759 & 5.16211 & -0.17689 \\
\hline 0 & -1.03662 & 5.13675 & 1.69145 \\
\hline $\mathrm{C}$ & 1.02321 & 6.34443 & 0.44253 \\
\hline $\mathrm{H}$ & 0.2571 & 6.99133 & 0.81595 \\
\hline $\mathrm{H}$ & 1.65447 & 6.03618 & 1.24963 \\
\hline $\mathrm{H}$ & 1.60637 & 6.86632 & -0.28714 \\
\hline 0 & 0.27231 & 0.85372 & 5.99165 \\
\hline 0 & 6.1323 & -0.81267 & 1.46256 \\
\hline $\mathrm{H}$ & 5.83928 & 0.91203 & 1.63027 \\
\hline $\mathrm{H}$ & 1.28301 & -1.04282 & 5.76061 \\
\hline $\mathrm{H}$ & -2.46311 & -1.4264 & 2.29053 \\
\hline $\mathrm{O}$ & -2.64775 & 0.2872 & 2.42239 \\
\hline
\end{tabular}




\begin{tabular}{|l|r|r|r|}
$\mathrm{O}$ & 2.64095 & -1.692 & -2.48701 \\
\hline $\mathrm{H}$ & 2.75008 & -0.03155 & -2.01199 \\
\hline
\end{tabular}

Table S3. Summary of optimization of macrocycle A

\begin{tabular}{|c|c|}
\hline File Name & Macrocycle A \\
\hline File Type & .log \\
\hline Calculation Type & FOPT \\
\hline Calculation Method & RB3LYP \\
\hline Basis Set & $6-31 G$ \\
\hline Charge & 0 \\
\hline Spin & Singlet \\
\hline E(RB3LYP) “Energy of & -3579.03 Hartree \\
\hline Optimized Structure” & 84 \\
\hline RMS Gradient Norm & 0.00000261 Hartree/Bohr \\
\hline Number Imaginary Freq. & C1 \\
\hline Dipole Moment & Pobye \\
\hline Point Group &
\end{tabular}

Table S4. Symbolic Z-matrix of macrocycle B, charge $=0$, multiplicity $=1$

\begin{tabular}{|l|r|r|r|}
\hline C & -1.84836 & 0.57776 & 2.39676 \\
\hline C & -0.49293 & 0.24197 & 2.28208 \\
\hline C & 0.48313 & 1.24547 & 2.34903 \\
\hline C & 0.10259 & 2.58665 & 2.50019 \\
\hline C & -1.25145 & 2.92481 & 2.58462 \\
\hline C & -2.22669 & 1.91799 & 2.55515 \\
\hline H & -2.59454 & -0.18848 & 2.36281 \\
\hline C & 1.84008 & 0.90605 & 2.27761 \\
\hline C & 1.07716 & 3.58599 & 2.59217 \\
\hline H & -3.2614 & 2.17341 & 2.65001 \\
\hline C & 2.43551 & 3.24104 & 2.57498 \\
\hline C & 2.81681 & 1.90198 & 2.41901 \\
\hline H & 3.18146 & 4.00092 & 2.67876 \\
\hline C & -0.07579 & -1.22899 & 2.08949 \\
\hline C & 1.27691 & -1.56041 & 1.9515 \\
\hline C & -1.05045 & -2.23574 & 2.06156 \\
\hline C & 2.25568 & -0.56199 & 2.05424 \\
\hline C & 1.65077 & -2.89205 & 1.72343 \\
\hline C & -0.67678 & -3.56785 & 1.84134 \\
\hline C & 3.61096 & -0.90249 & 1.95918 \\
\hline C & 3.0022 & -3.22647 & 1.59575 \\
\hline
\end{tabular}




\begin{tabular}{|c|c|c|c|}
\hline C & 0.67403 & -3.89199 & 1.64756 \\
\hline $\mathrm{H}$ & -1.42108 & -4.33654 & 1.81783 \\
\hline$C$ & 3.98401 & -2.23531 & 1.73629 \\
\hline $\mathrm{H}$ & 4.36095 & -0.1458 & 2.05594 \\
\hline $\mathrm{H}$ & 5.01949 & -2.49618 & 1.66941 \\
\hline C & 0.6679 & 5.06528 & 2.72252 \\
\hline 0 & 1.53888 & 5.92375 & 3.02016 \\
\hline$C$ & -1.67958 & 4.39856 & 2.71824 \\
\hline 0 & -2.87197 & 4.67063 & 3.01411 \\
\hline$C$ & 1.07416 & -5.35107 & 1.36157 \\
\hline 0 & 0.22309 & -6.26604 & 1.51162 \\
\hline$C$ & 3.42095 & -4.68066 & 1.30934 \\
\hline 0 & 4.63189 & -5.00662 & 1.41352 \\
\hline $\mathrm{N}$ & -0.72214 & 5.49641 & 2.49151 \\
\hline $\mathrm{N}$ & 2.43147 & -5.69394 & 0.90163 \\
\hline C & -1.03849 & 6.60992 & 3.39857 \\
\hline $\mathrm{H}$ & -0.35903 & 7.4177 & 3.22201 \\
\hline $\mathrm{H}$ & -2.04018 & 6.94135 & 3.22086 \\
\hline $\mathrm{H}$ & -0.94595 & 6.28153 & 4.41277 \\
\hline $\mathrm{C}$ & 2.41628 & -5.75529 & -0.567 \\
\hline $\mathrm{H}$ & 3.39101 & -6.01573 & -0.9231 \\
\hline $\mathrm{H}$ & 1.71094 & -6.49403 & -0.88562 \\
\hline $\mathrm{H}$ & 2.13589 & -4.80071 & -0.96097 \\
\hline 0 & 4.20053 & 1.54543 & 2.41357 \\
\hline 0 & -2.42126 & -1.88859 & 2.26784 \\
\hline$C$ & -0.29543 & -0.02963 & 6.74676 \\
\hline$C$ & 1.04855 & 0.35007 & 6.85448 \\
\hline C & 1.39898 & 1.70334 & 6.77396 \\
\hline$C$ & 0.40492 & 2.67667 & 6.5971 \\
\hline$C$ & -0.93773 & 2.29752 & 6.49949 \\
\hline$C$ & -1.29031 & 0.94459 & 6.59382 \\
\hline$C$ & 2.74283 & 2.08332 & 6.87576 \\
\hline$C$ & 0.75273 & 4.02932 & 6.54084 \\
\hline $\mathrm{H}$ & -2.31899 & 0.65527 & 6.54247 \\
\hline$C$ & 2.09351 & 4.41296 & 6.68085 \\
\hline$C$ & 3.0899 & 3.43998 & 6.83659 \\
\hline $\mathrm{H}$ & 2.35676 & 5.44974 & 6.66466 \\
\hline $\mathrm{H}$ & 4.11518 & 3.73325 & 6.92603 \\
\hline $\mathrm{C}$ & 2.141 & -0.71857 & 7.06076 \\
\hline C & 3.48857 & -0.34015 & 7.12396 \\
\hline$C$ & 1.78937 & -2.06829 & 7.18872 \\
\hline$C$ & 3.83777 & 1.01142 & 7.03433 \\
\hline$C$ & 4.48662 & -1.3118 & 7.28285 \\
\hline $\mathrm{C}$ & 2.78586 & -3.03754 & 7.3696 \\
\hline $\mathrm{H}$ & 0.76047 & -2.35931 & 7.14948 \\
\hline $\mathrm{C}$ & 5.18257 & 1.3931 & 7.08646 \\
\hline
\end{tabular}




\begin{tabular}{|c|c|c|c|}
\hline C & 5.83195 & -0.93058 & 7.34871 \\
\hline $\mathrm{C}$ & 4.13625 & -2.66074 & 7.3983 \\
\hline $\mathrm{H}$ & 2.51558 & -4.06687 & 7.48178 \\
\hline$C$ & 6.18034 & 0.42632 & 7.2673 \\
\hline $\mathrm{H}$ & 7.20646 & 0.7214 & 7.33716 \\
\hline$C$ & -0.32982 & 5.10432 & 6.3322 \\
\hline 0 & -0.03864 & 6.31572 & 6.50753 \\
\hline$C$ & -2.03468 & 3.35856 & 6.29193 \\
\hline 0 & -3.2428 & 3.03462 & 6.4321 \\
\hline$C$ & 5.22501 & -3.73718 & 7.56396 \\
\hline 0 & 4.89295 & -4.90618 & 7.89123 \\
\hline $\mathrm{C}$ & 6.93442 & -1.99319 & 7.51423 \\
\hline 0 & 8.10523 & -1.6293 & 7.79749 \\
\hline $\mathrm{N}$ & -1.69648 & 4.74294 & 5.91706 \\
\hline $\mathrm{N}$ & 6.64703 & -3.42772 & 7.33198 \\
\hline $\mathrm{C}$ & 6.97281 & -3.78875 & 5.94445 \\
\hline $\mathrm{H}$ & 8.00612 & -3.58459 & 5.75561 \\
\hline $\mathrm{H}$ & 6.78173 & -4.83069 & 5.79243 \\
\hline $\mathrm{H}$ & 6.36764 & -3.21402 & 5.27475 \\
\hline $\mathrm{C}$ & -2.65204 & 5.66258 & 6.55095 \\
\hline $\mathrm{H}$ & -2.42318 & 6.66775 & 6.26397 \\
\hline $\mathrm{H}$ & -3.6448 & 5.41873 & 6.23532 \\
\hline $\mathrm{H}$ & -2.58338 & 5.57102 & 7.6148 \\
\hline 0 & -0.64646 & -1.41317 & 6.77886 \\
\hline 0 & 5.51816 & 2.77327 & 6.93949 \\
\hline$C$ & -2.65613 & -1.8644 & 3.67712 \\
\hline$C$ & -3.94779 & -2.01959 & 4.19772 \\
\hline$C$ & -1.57222 & -1.67767 & 4.5403 \\
\hline $\mathrm{C}$ & -4.15285 & -1.95439 & 5.58537 \\
\hline $\mathrm{H}$ & -4.77545 & -2.18428 & 3.53982 \\
\hline$C$ & -1.77302 & -1.61331 & 5.92205 \\
\hline $\mathrm{H}$ & -0.58423 & -1.58084 & 4.14068 \\
\hline $\mathrm{C}$ & -3.06557 & -1.74175 & 6.44837 \\
\hline $\mathrm{H}$ & -5.13881 & -2.06616 & 5.98552 \\
\hline $\mathrm{H}$ & -3.22281 & -1.68036 & 7.50524 \\
\hline C & 5.58499 & 3.03649 & 5.53605 \\
\hline$C$ & 6.32128 & 4.11703 & 5.03105 \\
\hline $\mathrm{C}$ & 4.89392 & 2.19131 & 4.66137 \\
\hline $\mathrm{C}$ & 6.33928 & 4.36166 & 3.64804 \\
\hline $\mathrm{H}$ & 6.86524 & 4.75462 & 5.69663 \\
\hline $\mathrm{C}$ & 4.91501 & 2.42822 & 3.28433 \\
\hline $\mathrm{H}$ & 4.3447 & 1.35865 & 5.04916 \\
\hline C & 5.63029 & 3.52077 & 2.77429 \\
\hline $\mathrm{H}$ & 6.89394 & 5.19017 & 3.25941 \\
\hline $\mathrm{H}$ & 5.63775 & 3.7122 & 1.72134 \\
\hline
\end{tabular}


Table S5. Summary of optimization of macrocycle B

\begin{tabular}{|c|c|}
\hline File Name & Macrocycle B \\
\hline File Type & .log \\
\hline Calculation Type & FOPT \\
\hline Calculation Method & RB3LYP \\
\hline Basis Set & $6-31 \mathrm{G}$ \\
\hline Charge & 0 \\
\hline Spin & Singlet \\
\hline E(RB3LYP) “Energy of & -3579.03 Hartree \\
\hline Optimized Structure” & 0.00000152 Hartree/Bohr \\
\hline RMS Gradient Norm & 76 \\
\hline Number Imaginary Freq & 0.0841 Debye \\
\hline Dipole Moment & C1 \\
\hline Point Group &
\end{tabular}
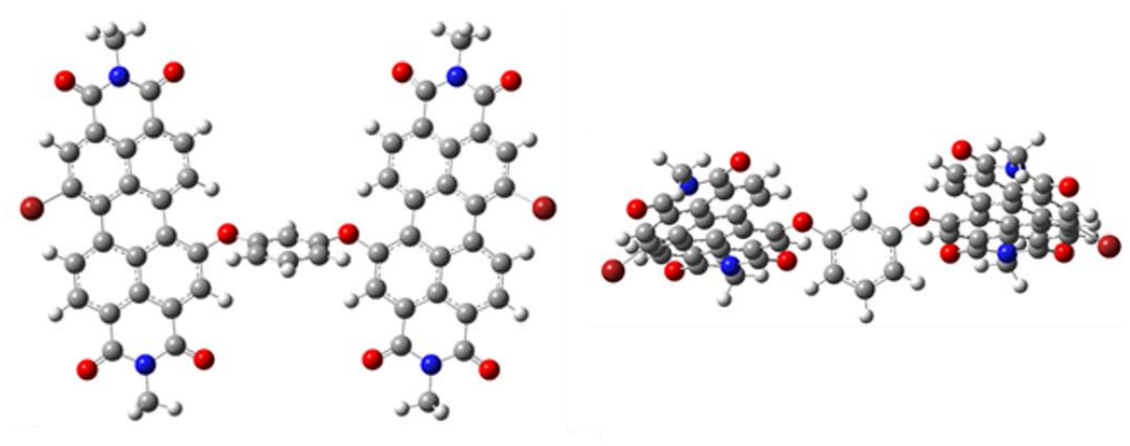

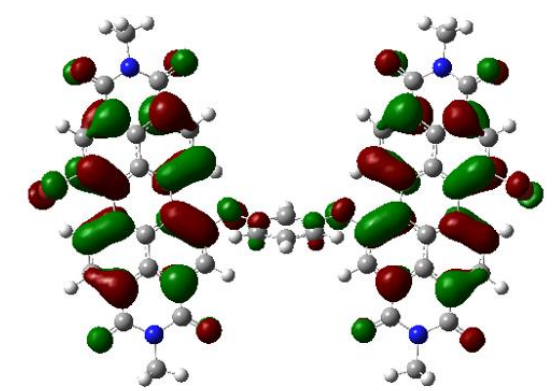

HOMO: $-6.20 \mathrm{eV}$

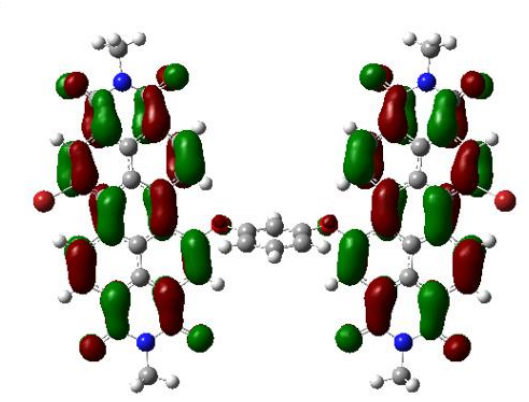

LUMO: $-3.71 \mathrm{eV}$

Figure S16. 3D Structures of the lowest energy conformers and frontier orbitals of $\mathrm{PBI}_{2} \mathrm{RBr}_{2}$ obtained by DFT at B3LYP/6-31G(d) Level

Table S6. Symbolic Z-matrix of $\mathrm{PBI}_{2} \mathrm{RBr}_{2}:$ Charge $=0$ Multiplicity $=1$

\begin{tabular}{|l|r|r|r|}
\hline C & -4.26774 & -1.30159 & 1.17383 \\
\hline C & -2.89443 & -1.55017 & 1.03097 \\
\hline C & -2.0163 & -0.4804 & 0.81843 \\
\hline C & -2.50754 & 0.82908 & 0.70985 \\
\hline
\end{tabular}




\begin{tabular}{|c|c|c|c|}
\hline C & -3.8775 & 1.07765 & 0.82855 \\
\hline C & -4.75719 & 0.01322 & 1.08568 \\
\hline$C$ & -0.64278 & -0.71773 & 0.72186 \\
\hline C & -1.62246 & 1.89153 & 0.50601 \\
\hline $\mathrm{H}$ & -5.8035 & 0.20277 & 1.21066 \\
\hline $\mathrm{C}$ & -0.24437 & 1.65272 & 0.45124 \\
\hline C & 0.24367 & 0.34753 & 0.55113 \\
\hline $\mathrm{H}$ & 0.43535 & 2.46936 & 0.32812 \\
\hline $\mathrm{H}$ & 1.29554 & 0.1624 & 0.49537 \\
\hline C & -2.34397 & -2.99361 & 1.11254 \\
\hline C & -0.967 & -3.22515 & 0.9763 \\
\hline $\mathrm{C}$ & -3.21164 & -4.07397 & 1.32499 \\
\hline$C$ & -0.09513 & -2.14761 & 0.80489 \\
\hline C & -0.46025 & -4.53046 & 1.01925 \\
\hline$C$ & -2.70205 & -5.38114 & 1.38249 \\
\hline $\mathrm{H}$ & -4.26178 & -3.9031 & 1.44361 \\
\hline $\mathrm{C}$ & 1.28233 & -2.35641 & 0.70598 \\
\hline $\mathrm{C}$ & 0.91848 & -4.75099 & 0.89322 \\
\hline C & -1.32784 & -5.60985 & 1.21211 \\
\hline $\mathrm{H}$ & -3.36367 & -6.20523 & 1.55341 \\
\hline C & 1.79553 & -3.66047 & 0.75558 \\
\hline $\mathrm{H}$ & 2.8502 & -3.82513 & 0.68654 \\
\hline 0 & 2.14 & -1.21771 & 0.55029 \\
\hline C & 3.23937 & -1.51006 & -0.31592 \\
\hline $\mathrm{C}$ & 3.13141 & -2.53841 & -1.25968 \\
\hline C & 4.42315 & -0.759 & -0.22585 \\
\hline C & 4.19885 & -2.80873 & -2.12544 \\
\hline $\mathrm{H}$ & 2.23169 & -3.11575 & -1.32071 \\
\hline $\mathrm{C}$ & 5.48453 & -1.01495 & -1.10873 \\
\hline $\mathrm{H}$ & 4.51315 & 0.0127 & 0.51096 \\
\hline$C$ & 5.37381 & -2.04646 & -2.05187 \\
\hline $\mathrm{H}$ & 4.1168 & -3.5968 & -2.84344 \\
\hline $\mathrm{H}$ & 6.18551 & -2.25009 & -2.71941 \\
\hline 0 & 6.6775 & -0.22106 & -1.05839 \\
\hline C & 10.26243 & -2.04254 & -5.69414 \\
\hline C & 9.80715 & -1.98403 & -4.37286 \\
\hline $\mathrm{C}$ & 8.79952 & -1.07877 & -4.01749 \\
\hline C & 8.2831 & -0.19707 & -4.97758 \\
\hline $\mathrm{C}$ & 8.75101 & -0.24426 & -6.29468 \\
\hline C & 9.72463 & -1.18261 & -6.65796 \\
\hline $\mathrm{H}$ & 11.02028 & -2.74617 & -5.9673 \\
\hline$C$ & 8.30173 & -1.06509 & -2.70584 \\
\hline C & 7.28229 & 0.71254 & -4.62724 \\
\hline $\mathrm{H}$ & 10.0612 & -1.23887 & -7.67203 \\
\hline C & 6.7594 & 0.70696 & -3.32534 \\
\hline$C$ & 7.25308 & -0.19344 & -2.36934 \\
\hline
\end{tabular}




\begin{tabular}{|c|c|c|c|}
\hline $\mathrm{H}$ & 5.98039 & 1.39019 & -3.06064 \\
\hline$C$ & 10.41167 & -2.92183 & -3.31676 \\
\hline$C$ & 9.94855 & -2.88288 & -1.9963 \\
\hline C & 11.42377 & -3.8192 & -3.68044 \\
\hline $\mathrm{C}$ & 8.90801 & -2.01053 & -1.64519 \\
\hline $\mathrm{C}$ & 10.52085 & -3.72443 & -1.03103 \\
\hline C & 11.98715 & -4.66591 & -2.71854 \\
\hline$C$ & 8.42107 & -2.00357 & -0.33139 \\
\hline C & 10.04993 & -3.69967 & 0.28396 \\
\hline $\mathrm{C}$ & 11.54809 & -4.60581 & -1.38956 \\
\hline $\mathrm{H}$ & 12.7563 & -5.35542 & -2.99746 \\
\hline$C$ & 8.98746 & -2.85333 & 0.63016 \\
\hline $\mathrm{H}$ & 7.61833 & -1.35025 & -0.06083 \\
\hline $\mathrm{H}$ & 8.61167 & -2.85245 & 1.63188 \\
\hline $\mathrm{Br}$ & -5.46929 & -2.7525 & 1.4855 \\
\hline $\mathrm{Br}$ & 12.03328 & -3.88747 & -5.4906 \\
\hline $\mathrm{C}$ & -2.14731 & 3.32705 & 0.34685 \\
\hline 0 & -1.33523 & 4.28806 & 0.38427 \\
\hline$C$ & -4.42229 & 2.51334 & 0.68388 \\
\hline 0 & -5.60844 & 2.76086 & 1.02491 \\
\hline $\mathrm{C}$ & -0.7816 & -7.05033 & 1.24313 \\
\hline 0 & -1.52294 & -7.98494 & 1.64634 \\
\hline$C$ & 1.47874 & -6.18575 & 0.91204 \\
\hline 0 & 2.71914 & -6.36131 & 1.0164 \\
\hline $\mathrm{C}$ & 6.73816 & 1.71345 & -5.66681 \\
\hline 0 & 5.71314 & 2.38919 & -5.39132 \\
\hline$C$ & 8.19601 & 0.72279 & -7.35565 \\
\hline 0 & 8.43955 & 0.50857 & -8.57127 \\
\hline $\mathrm{C}$ & 10.68444 & -4.60431 & 1.35549 \\
\hline 0 & 10.11275 & -4.73391 & 2.46982 \\
\hline$C$ & 12.19618 & -5.51955 & -0.33253 \\
\hline 0 & 12.95467 & -6.45368 & -0.70488 \\
\hline $\mathrm{N}$ & -3.57978 & 3.59576 & 0.13545 \\
\hline $\mathrm{N}$ & 0.59006 & -7.35688 & 0.79713 \\
\hline $\mathrm{N}$ & 11.95589 & -5.30918 & 1.10731 \\
\hline$N$ & 7.39925 & 1.90178 & -6.97268 \\
\hline$C$ & -3.92418 & 4.87099 & 0.78076 \\
\hline $\mathrm{H}$ & -3.32907 & 5.65587 & 0.35884 \\
\hline $\mathrm{H}$ & -4.96043 & 5.08353 & 0.61931 \\
\hline $\mathrm{H}$ & -3.73227 & 4.80407 & 1.8297 \\
\hline $\mathrm{C}$ & 0.53319 & -7.75594 & -0.61837 \\
\hline $\mathrm{H}$ & 0.13855 & -6.94883 & -1.20034 \\
\hline $\mathrm{H}$ & 1.51733 & -7.99479 & -0.96329 \\
\hline $\mathrm{H}$ & -0.09904 & -8.6146 & -0.72191 \\
\hline$C$ & 8.31156 & 3.05097 & -6.87677 \\
\hline $\mathrm{H}$ & 8.79286 & 3.20256 & -7.82146 \\
\hline
\end{tabular}




\begin{tabular}{|l|r|r|r|}
$\mathrm{H}$ & 7.757 & 3.92878 & -6.61346 \\
\hline $\mathrm{H}$ & 9.04992 & 2.85761 & -6.12847 \\
\hline $\mathrm{C}$ & 11.92072 & -6.61113 & 1.78762 \\
\hline $\mathrm{H}$ & 12.84687 & -7.1218 & 1.62656 \\
\hline $\mathrm{H}$ & 11.77334 & -6.46216 & 2.83666 \\
\hline $\mathrm{H}$ & 11.11672 & -7.19689 & 1.39481 \\
\hline
\end{tabular}

Table S7. Summary of optimization of $\mathrm{PBI}_{2} \mathrm{RBr}_{2}$

\begin{tabular}{|c|c|}
\hline File Name & $\mathrm{PBI}_{2} \mathrm{RBr}_{2}$ \\
\hline File Type &. $\log$ \\
\hline Calculation Type & FOPT \\
\hline Calculation Method & RB3LYP \\
\hline Basis Set & $6-31 \mathrm{G}$ \\
\hline Charge & 0 \\
\hline Spin & Singlet \\
\hline E(RB3LYP) “Energy of & -8340.80 Hartree \\
\hline Optimized Structure” & 82 \\
\hline RMS Gradient Norm & 0.00000084 Hartree/Bohr \\
\hline Number Imaginary Freq & 1.5145 \\
\hline Dipole Moment & $\mathrm{C} 1$ \\
\hline Point Group &
\end{tabular}
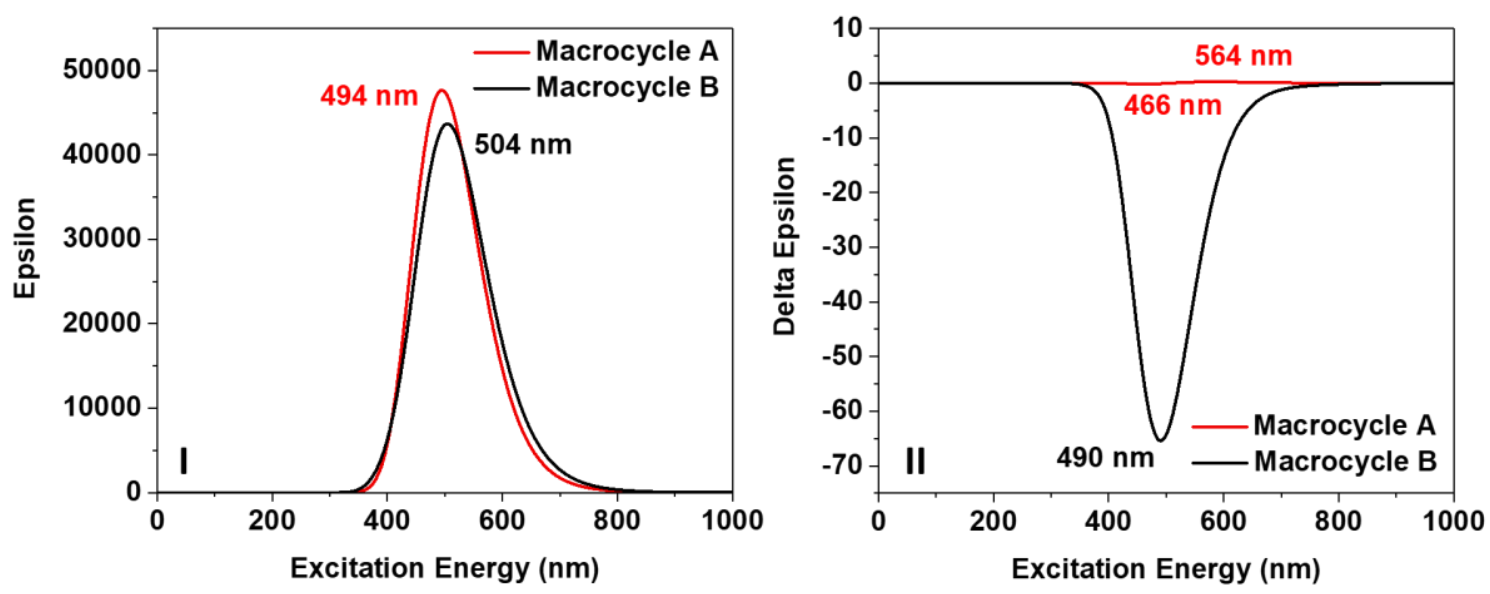

Figure S17. Calculated UV-Vis (I) and CD spectra (II) of the macrocycles from TD-DFT modelling 


\section{3. Absorbance and Fluorescence Properties}
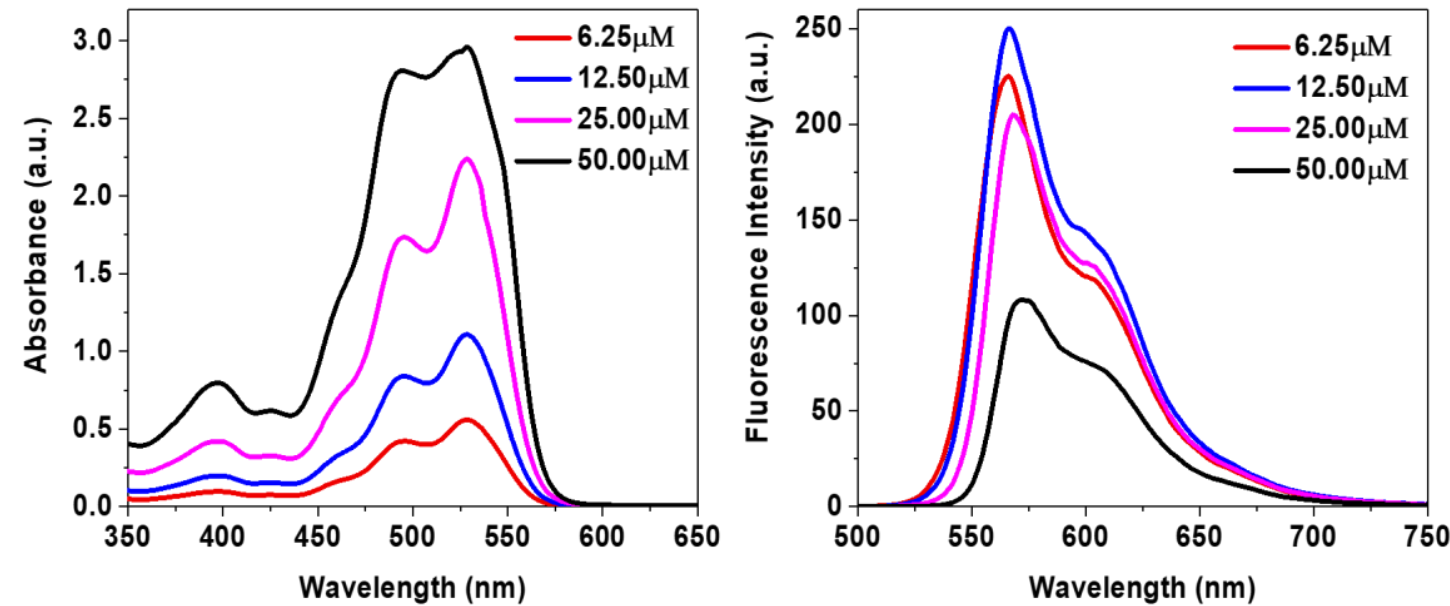

Figure S18. Concentration dependent UV-Vis and fluorescence spectra of $\mathrm{PBI}_{2} \mathrm{RBr}_{2}$ in $\mathrm{CHCl}_{3}$
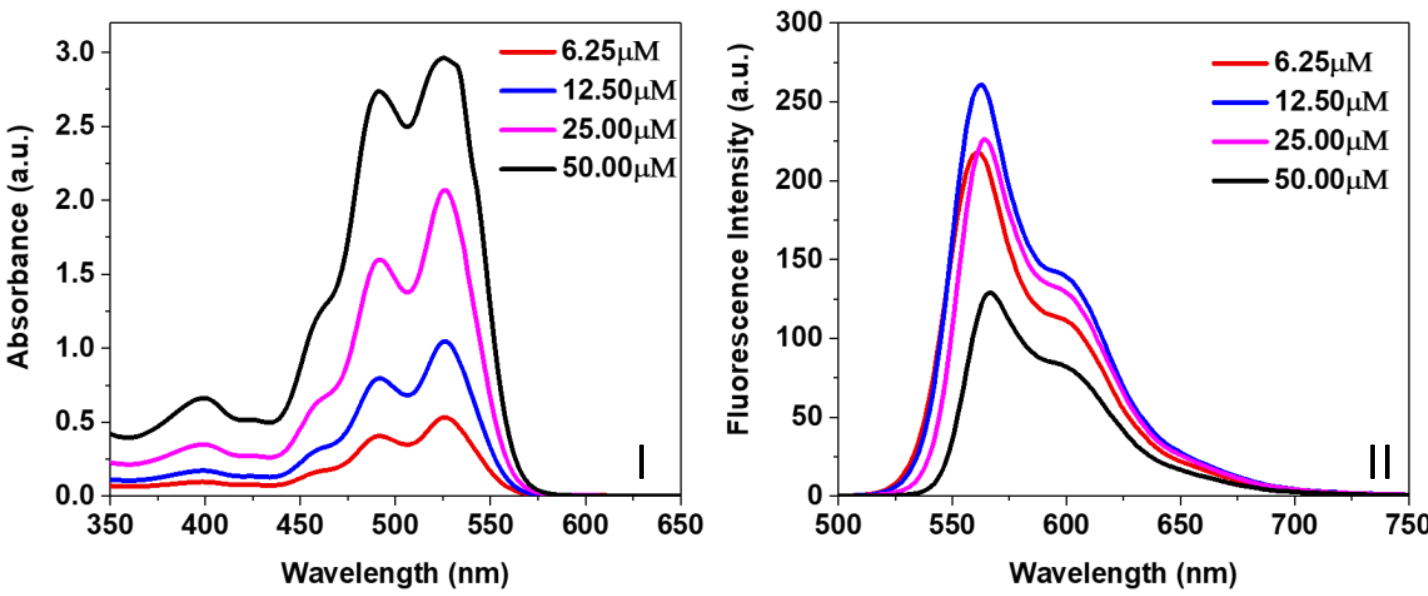

Figure S19. Concentration dependent UV-Vis (I)and fluorescence (II) spectra of $\mathrm{PBI}_{2} \mathrm{RBr}_{2}$ in toluene
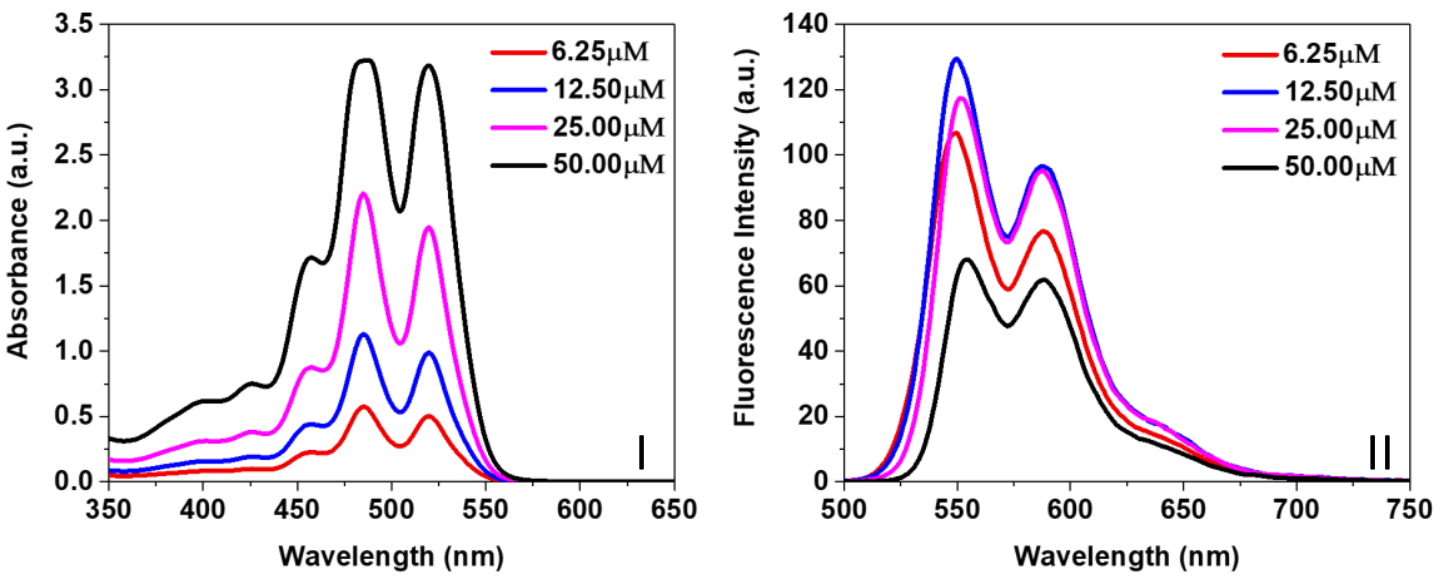

Figure S20. Concentration dependent UV-Vis (I) and fluorescence (II) spectra of macrocycle $\mathrm{A}$ in $\mathrm{CHCl}_{3}$ 

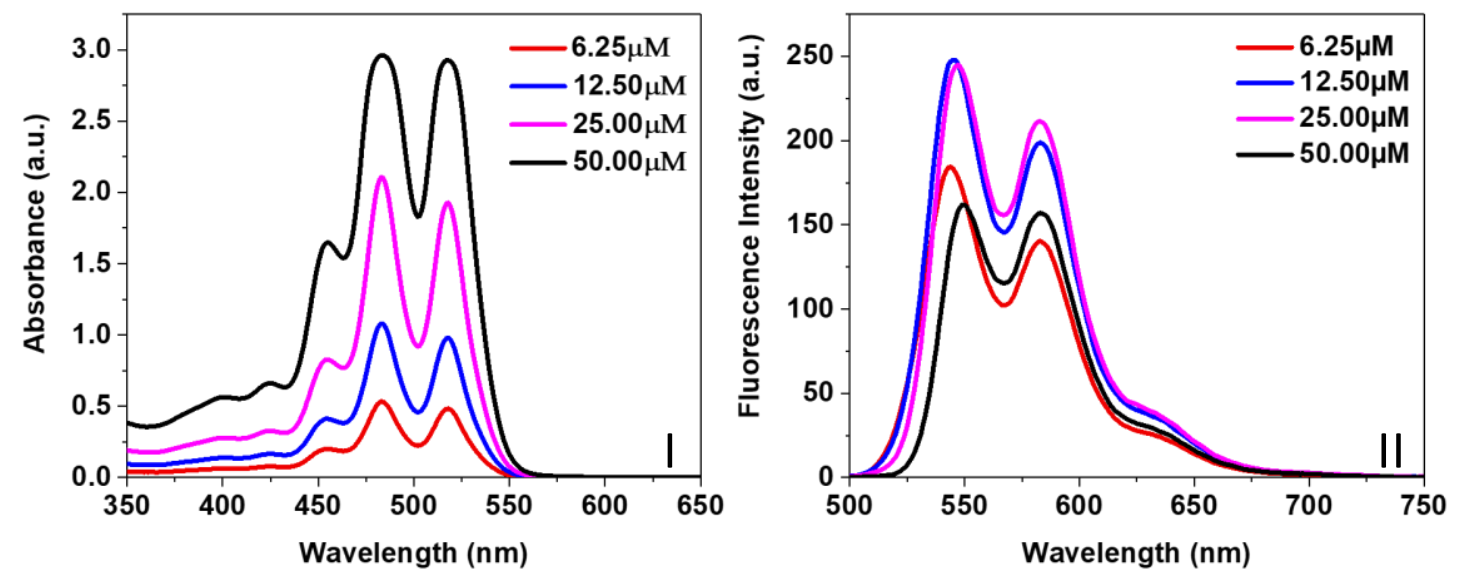

Figure S21. Concentration dependent UV-Vis (I) and fluorescence (II) spectra of macrocycle $A$ in toluene
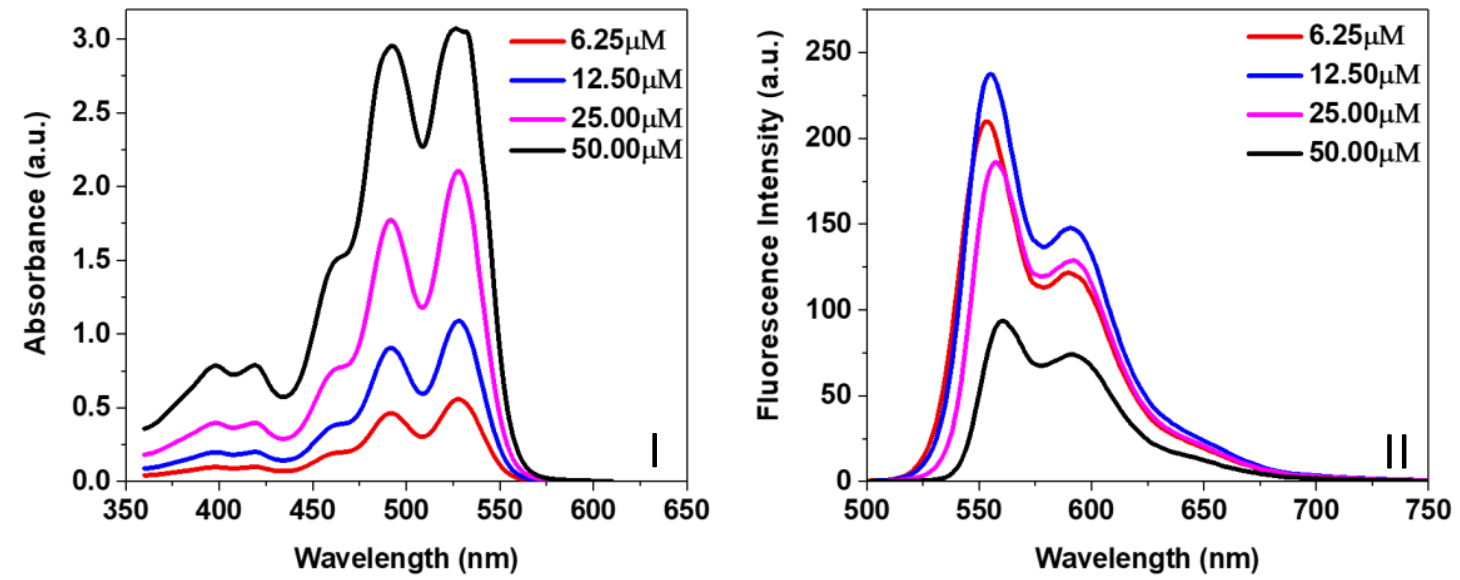

Figure S22. Concentration dependent UV-Vis (I) and fluorescence (II) spectra of macrocycle B in $\mathrm{CHCl}_{3}$
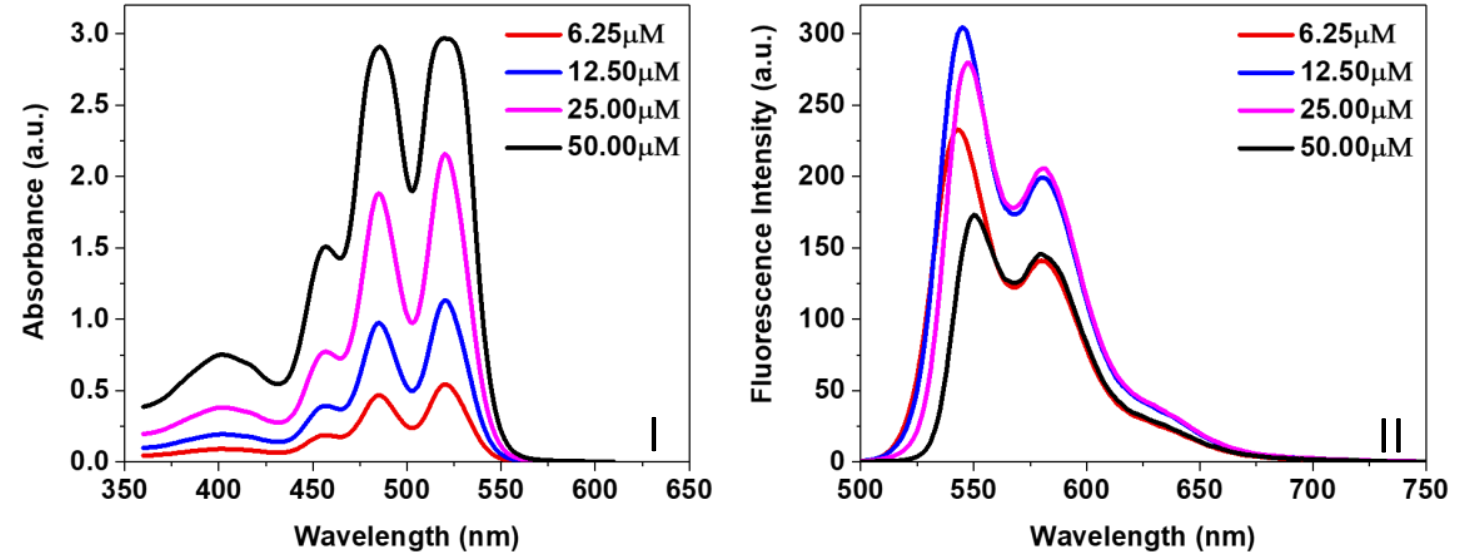

Figure S23. Concentration dependent UV-Vis (I) and fluorescence (II) spectra of macrocycle B in toluene 

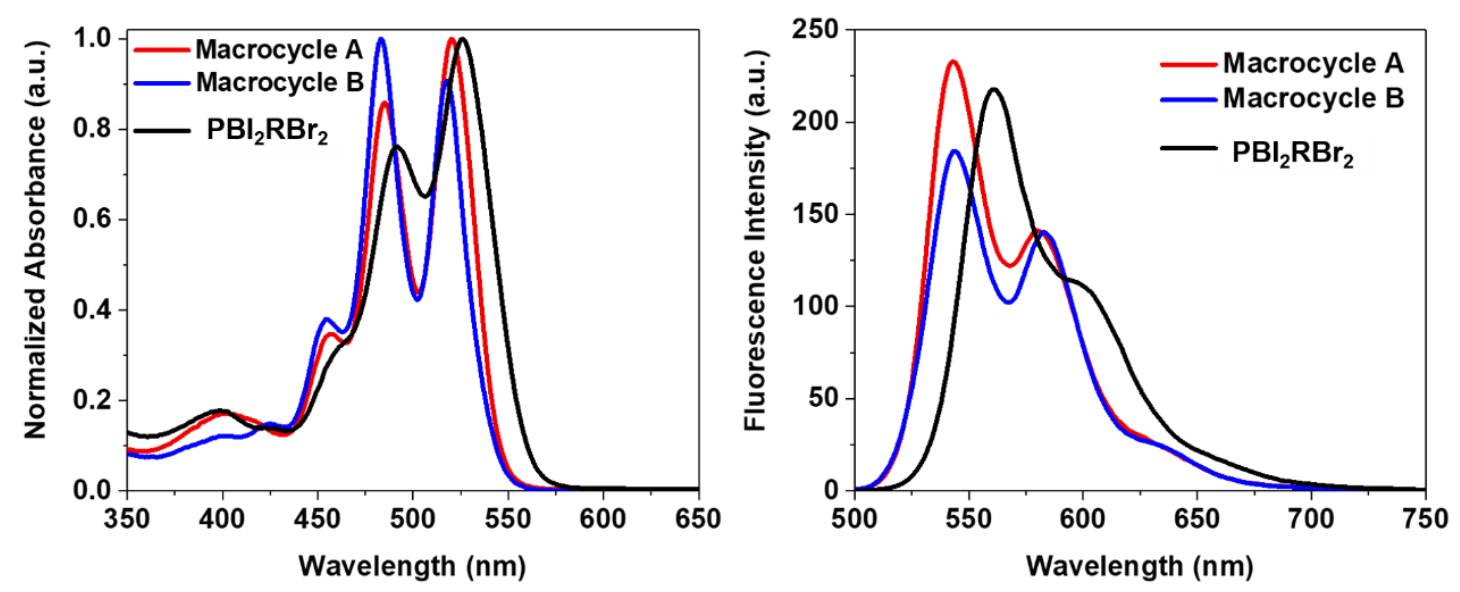

Figure S24. UV-Vis and fluorescence spectra of the cyclic and linear dimers in toluene
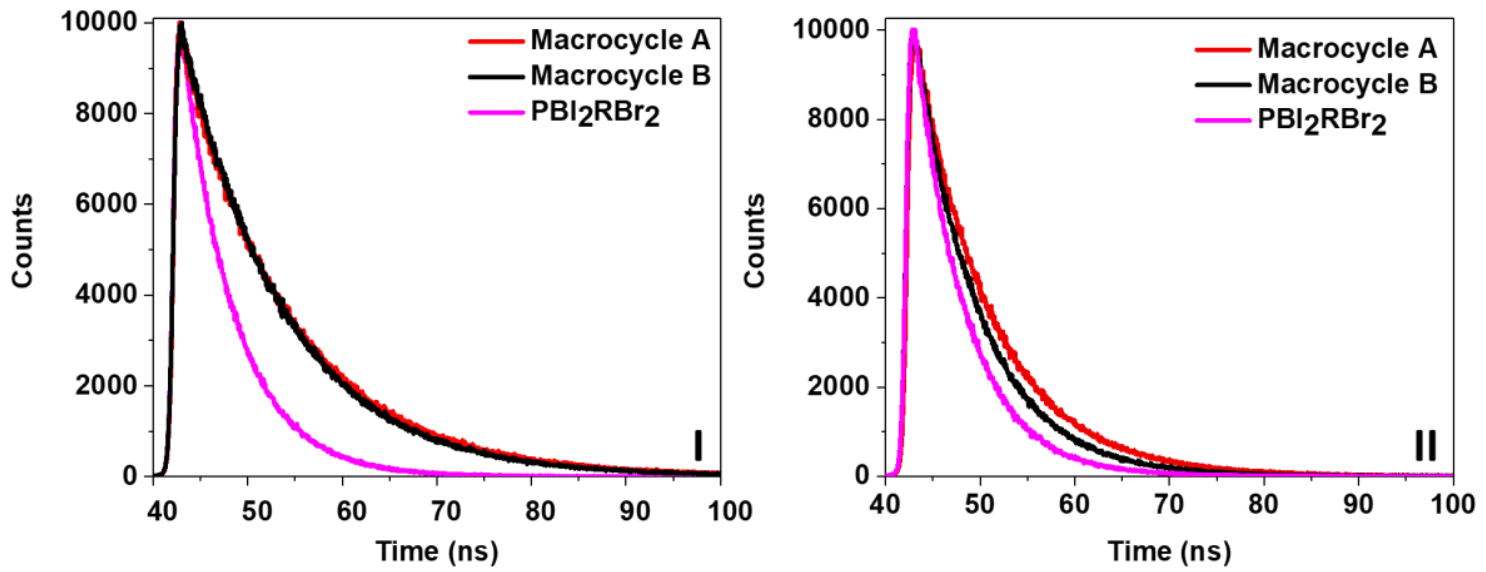

Figure S25. TCSPC lifetime of macrocycles vs $\mathrm{PBI}_{2} \mathrm{RBr}_{2}$ in chloroform (I) and in toluene (II)
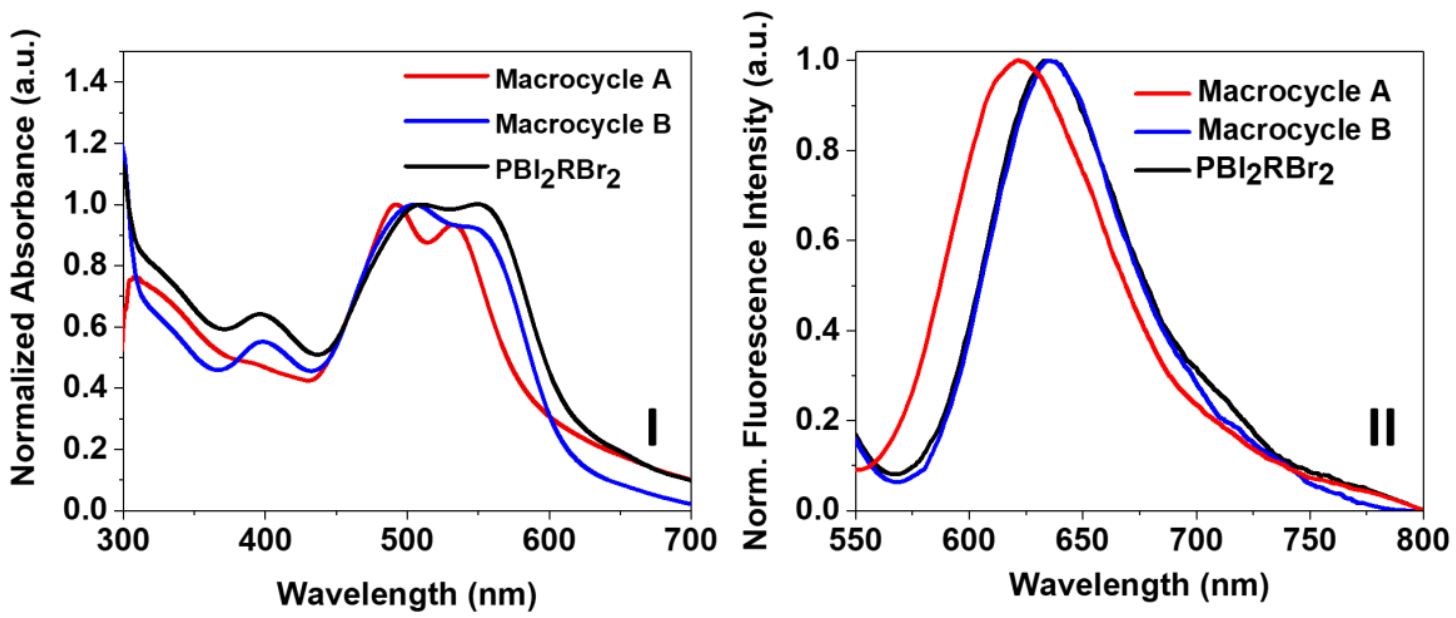

Figure S26. Absorbance (I) and emission (II) of the target Compounds in solid state 


\section{4. Electrochemistry}
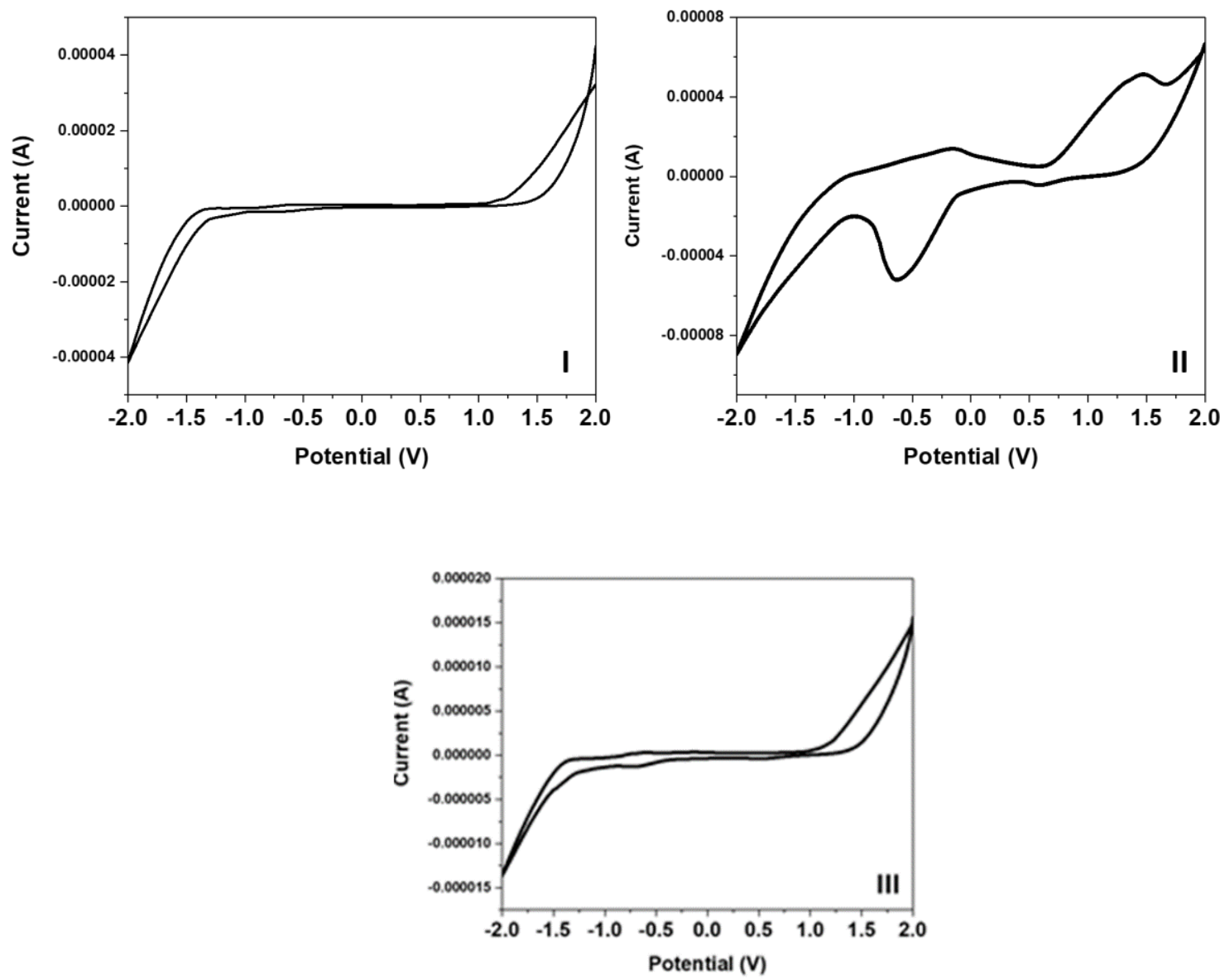

Figure S27. Cyclic voltammetry of macrocycle A (I), macrocycle B (II), $\mathrm{PBI}_{2} \mathrm{RBr}_{2}$ (III). 

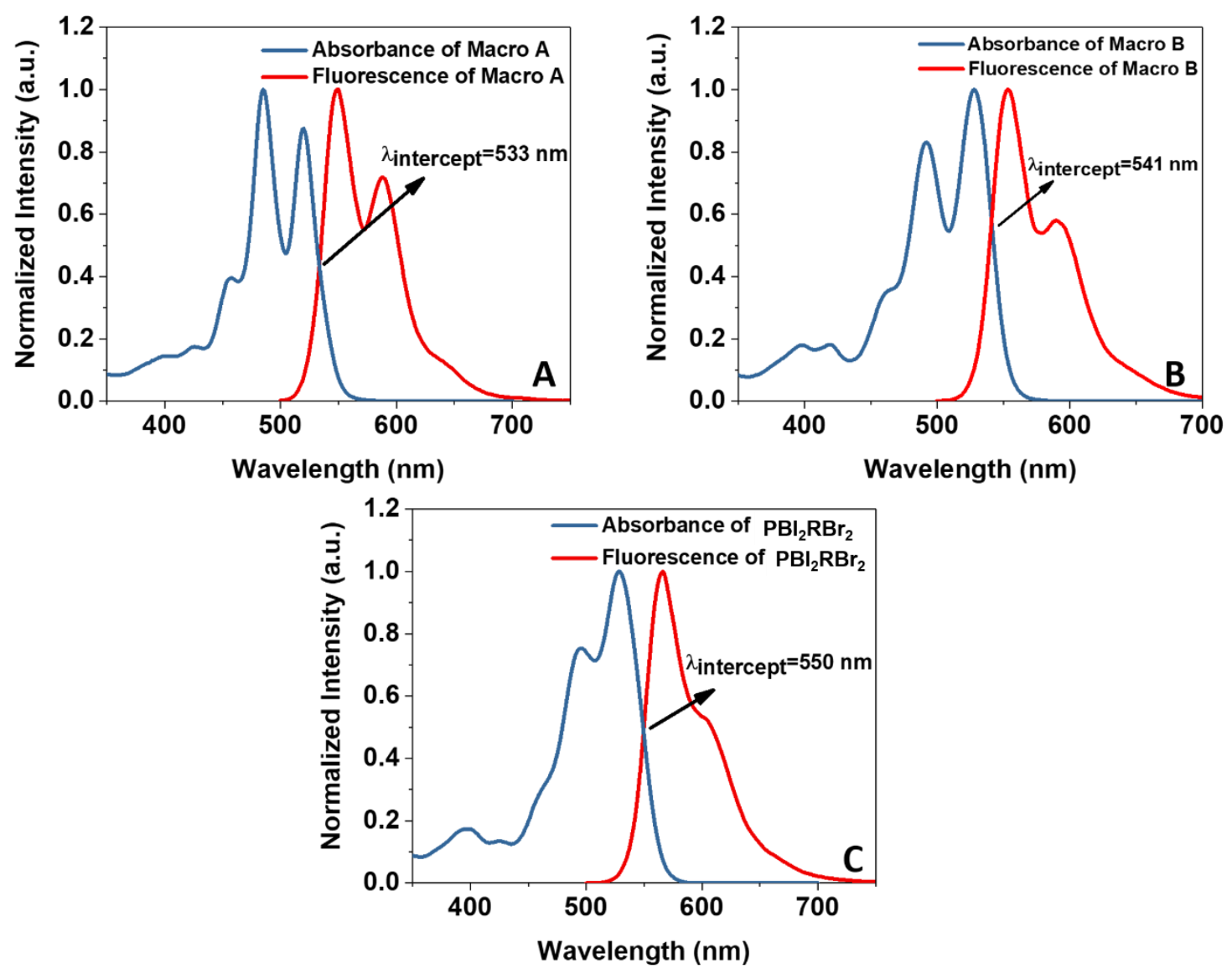

Figure S28. Absorbance-fluorescence interception wavelengths of macrocycles $A(A), B(B)$ and linear compound, $\mathrm{PBI}_{2} \mathrm{RBr}_{2}$ (C).

\section{5. UV-Vis and Fluorescence Titrations of Macrocycles with Azobenzene Derivatives}
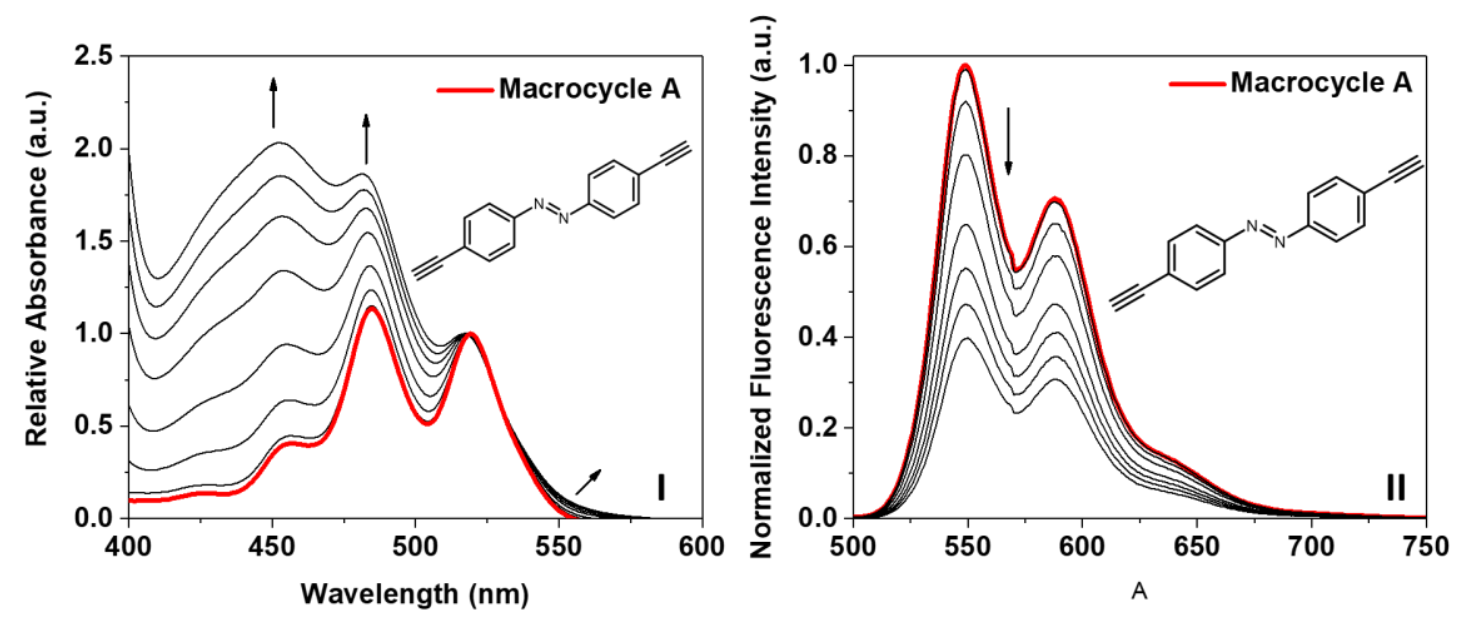

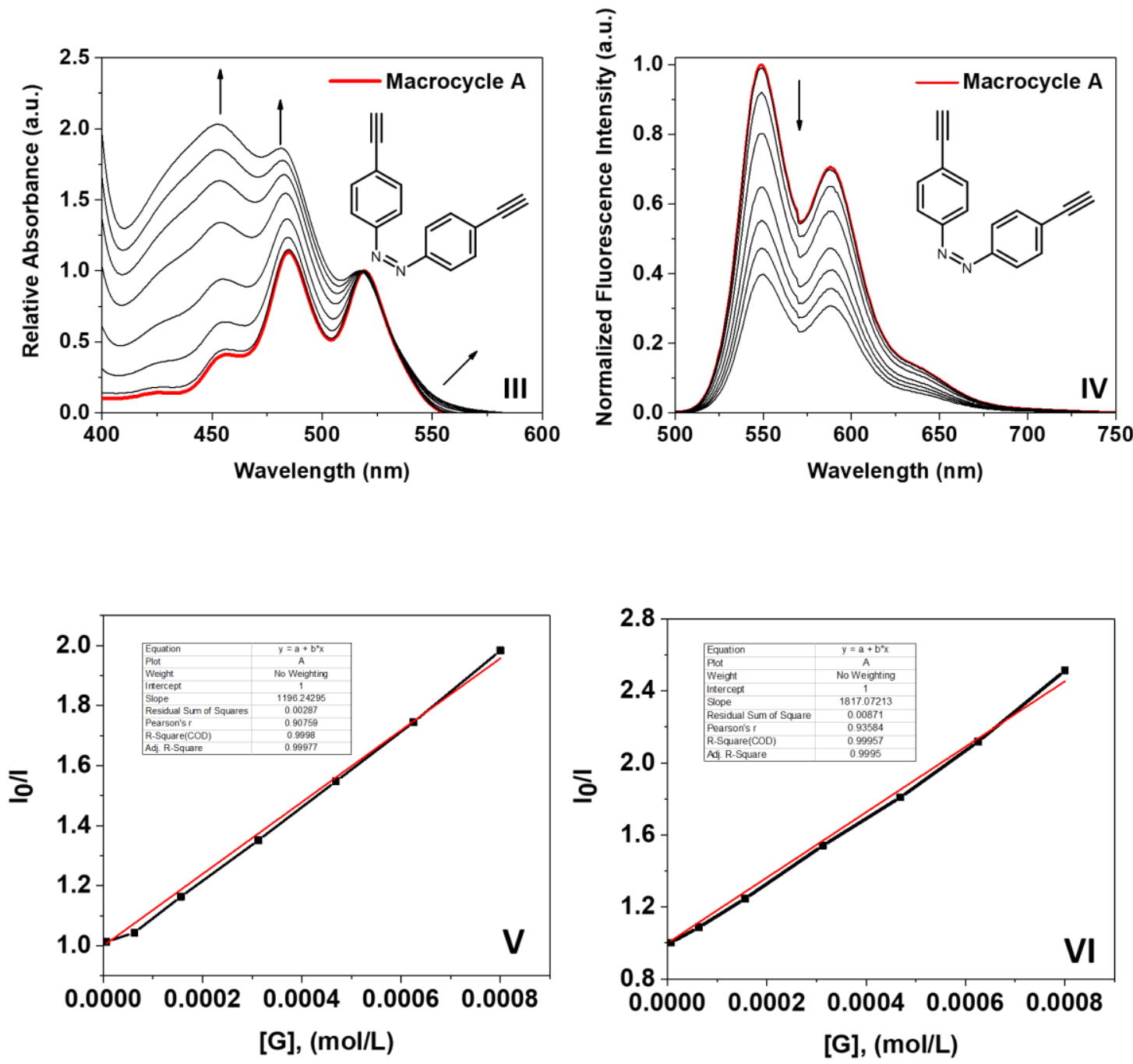

Figure S29. Titration of macrocycle A with $E$ - and Z- 1,2-bis(4-ethynylphenyl)-diazene in $\mathrm{CHCl}_{3}$. Absorption (I, III) and emission (II, IV) spectra of macrocycle A with E- and Z- 1,2-bis(4ethynylphenyl)-diazene. Stern-Volmer graphs of fluorescence titrations of macrocycle $A$ with different concentrations of $E$ - 1,2-bis(4-ethynylphenyl)-diazene (V), Z- 1,2-bis(4ethynylphenyl)diazene (VI). 

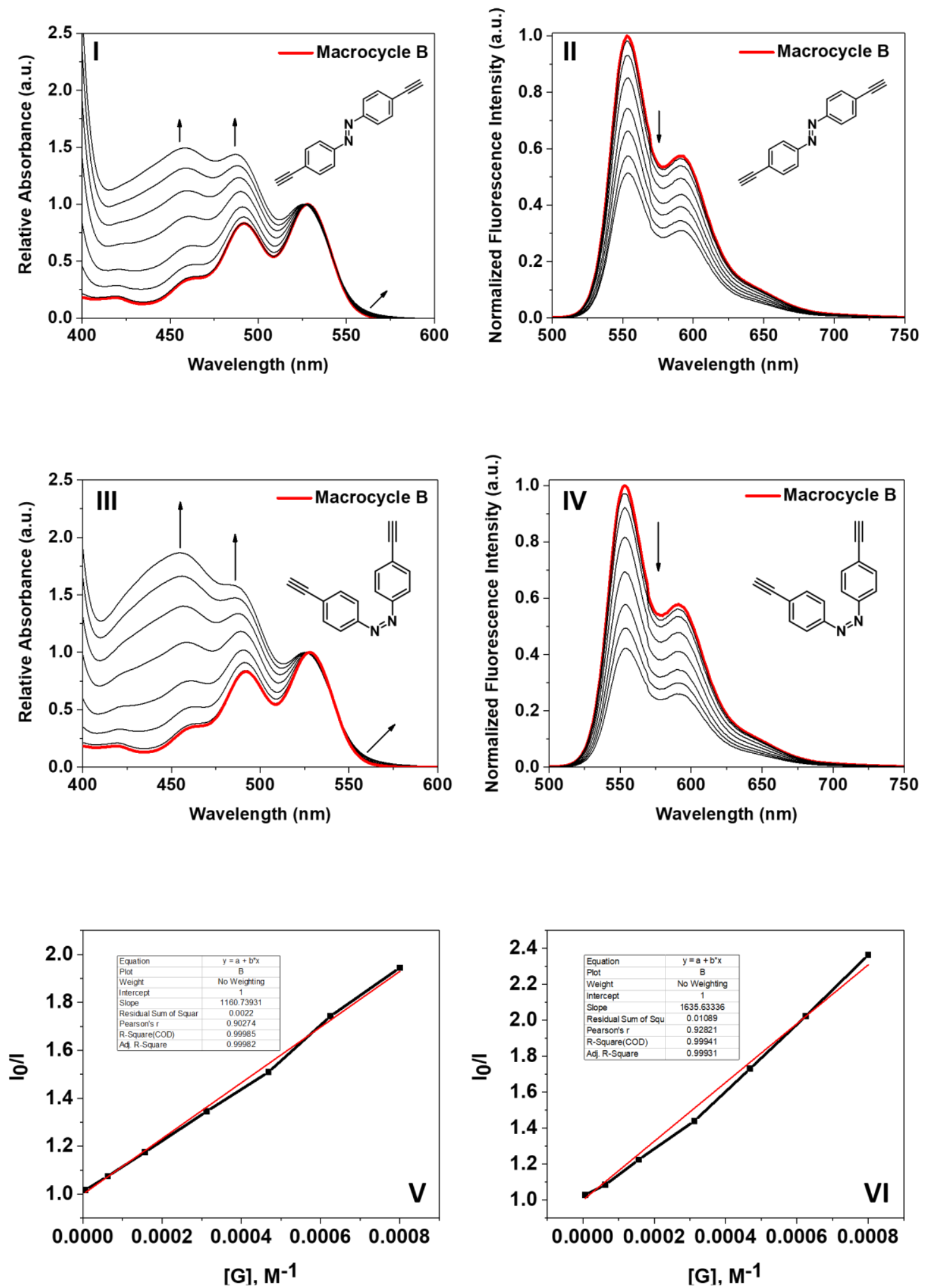

Figure S30. Titration of macrocycle B with $E$ - and Z- 1,2-bis(4-ethynylphenyl)-diazene in $\mathrm{CHCl}_{3}$. Absorption (I, III) and emission (II, IV) spectra of macrocycle A with E- and Z- 1,2-bis(4ethynylphenyl)diazene. Stern-Volmer graphs of fluorescence titrations of macrocycle $B$ with E- 1,2-bis(4-ethynylphenyl)-diazene (V), Z- 1,2-bis(4-ethynylphenyl)diazene (VI). 

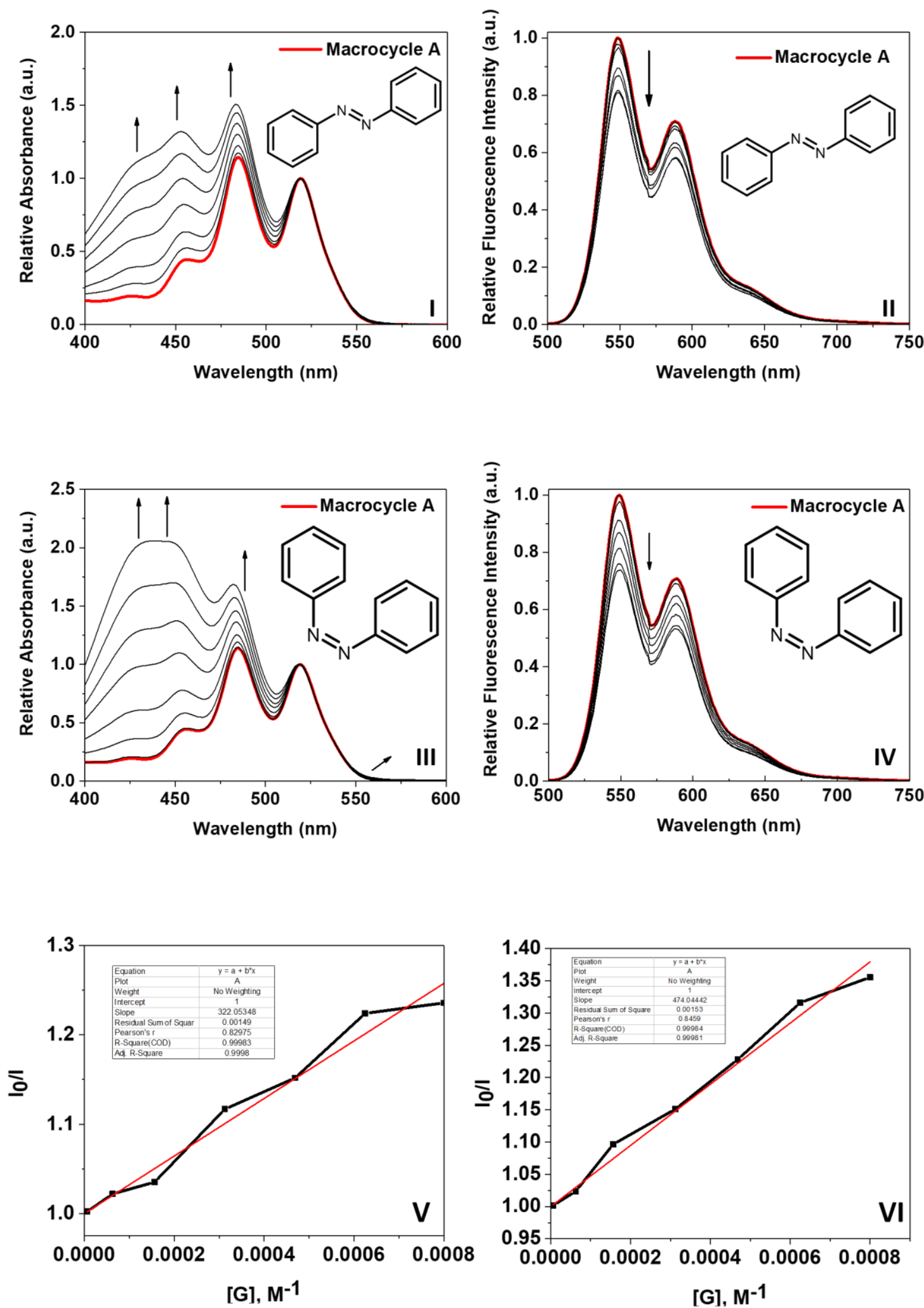

Figure S31. Titration of macrocycle A with $E$ - and Z- azobenzene in $\mathrm{CHCl}_{3}$. Absorption (I, III) and emission (II, IV) spectra of macrocycle $A$ in presence of $E$ - and Z- azobenzene. SternVolmer graphs of fluorescence titrations of macrocycle $A$ with $E$ - azobenzene (V), Zazobenzene (VI). 

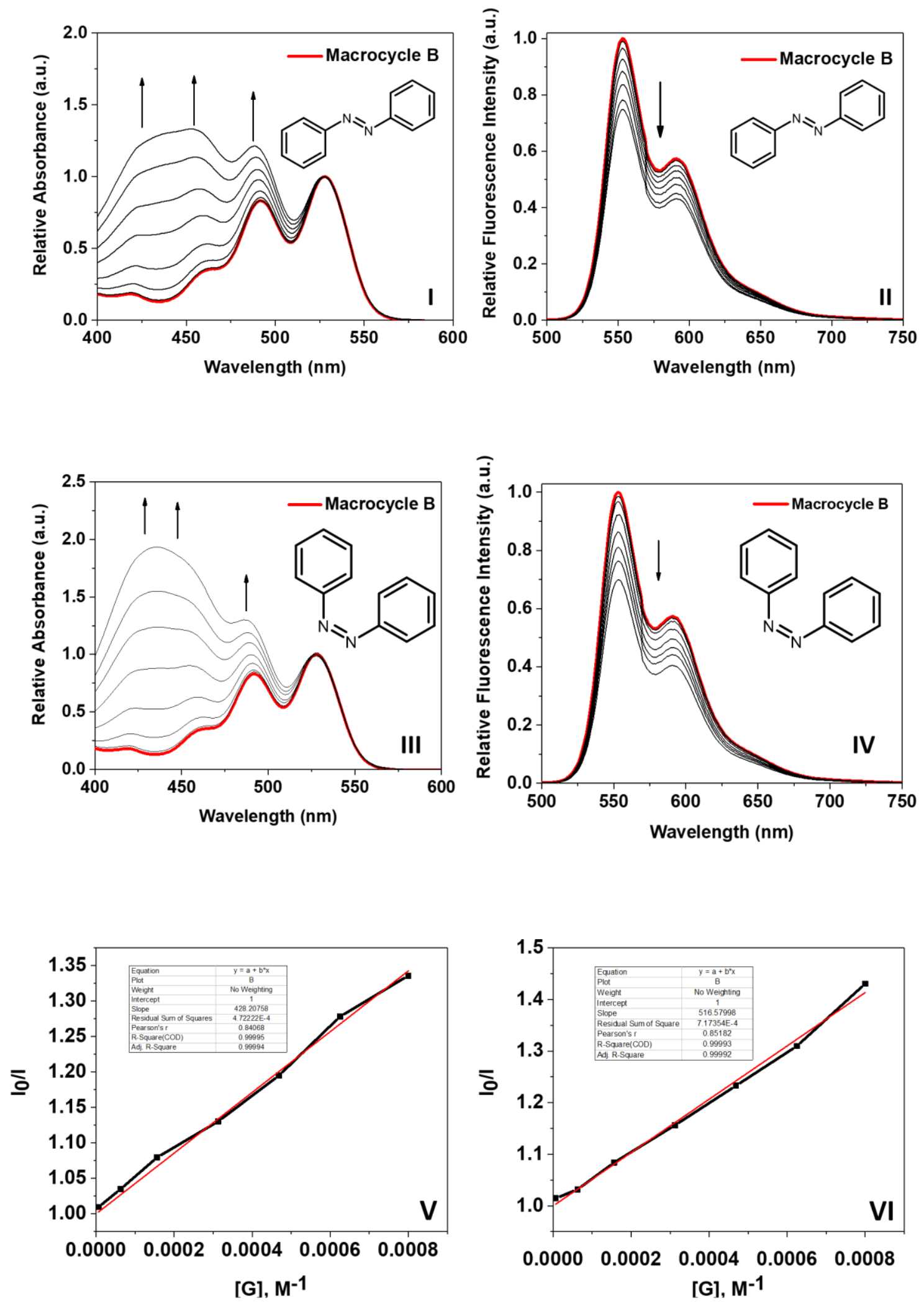

Figure S32. Titration of macrocycle B with $E$ - and Z- azobenzene in $\mathrm{CHCl}_{3}$. Absorption (I, III) and emission (II, IV) spectra of macrocycle B with $E$ - and Z- azobenzene. Stern-Volmer graphs of fluorescence titrations of macrocycle $B$ with $E$ - azobenzene (V), Z- azobenzene (VI). 

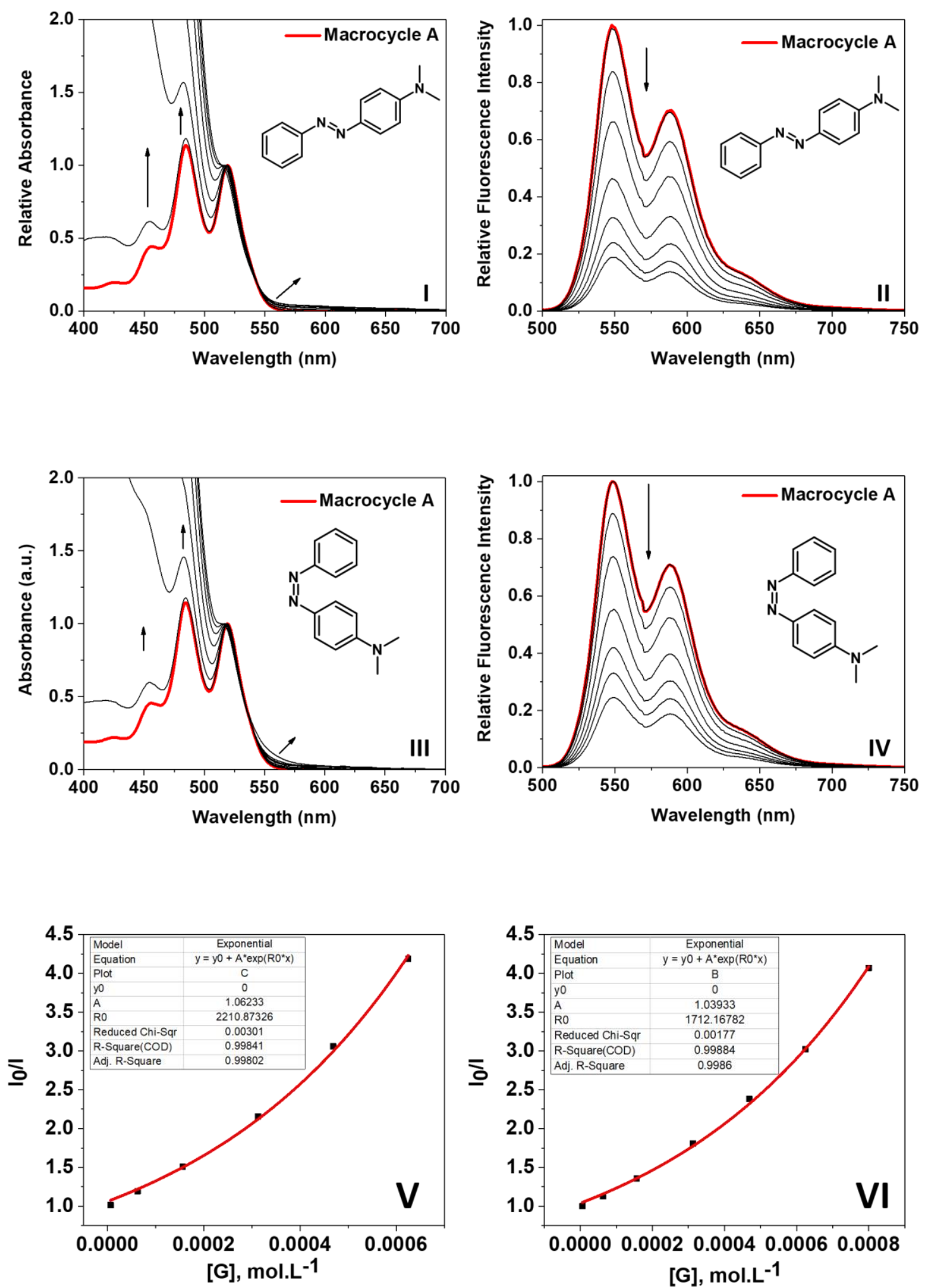

Figure S33. Titration of macrocycle A with $E$ - and Z- dimethylaminoazobenzene in $\mathrm{CHCl}_{3}$. Absorption (I, III) and emission (II, IV) spectra of macrocycle A with $E$ - and Z- 4dimethylaminoazobenzene. Stern-Volmer graphs of fluorescence titrations of macrocycle $A$ with E- 4-dimethylaminoazobenzene (V), Z- 4-dimethylaminoazobenzene (VI). 

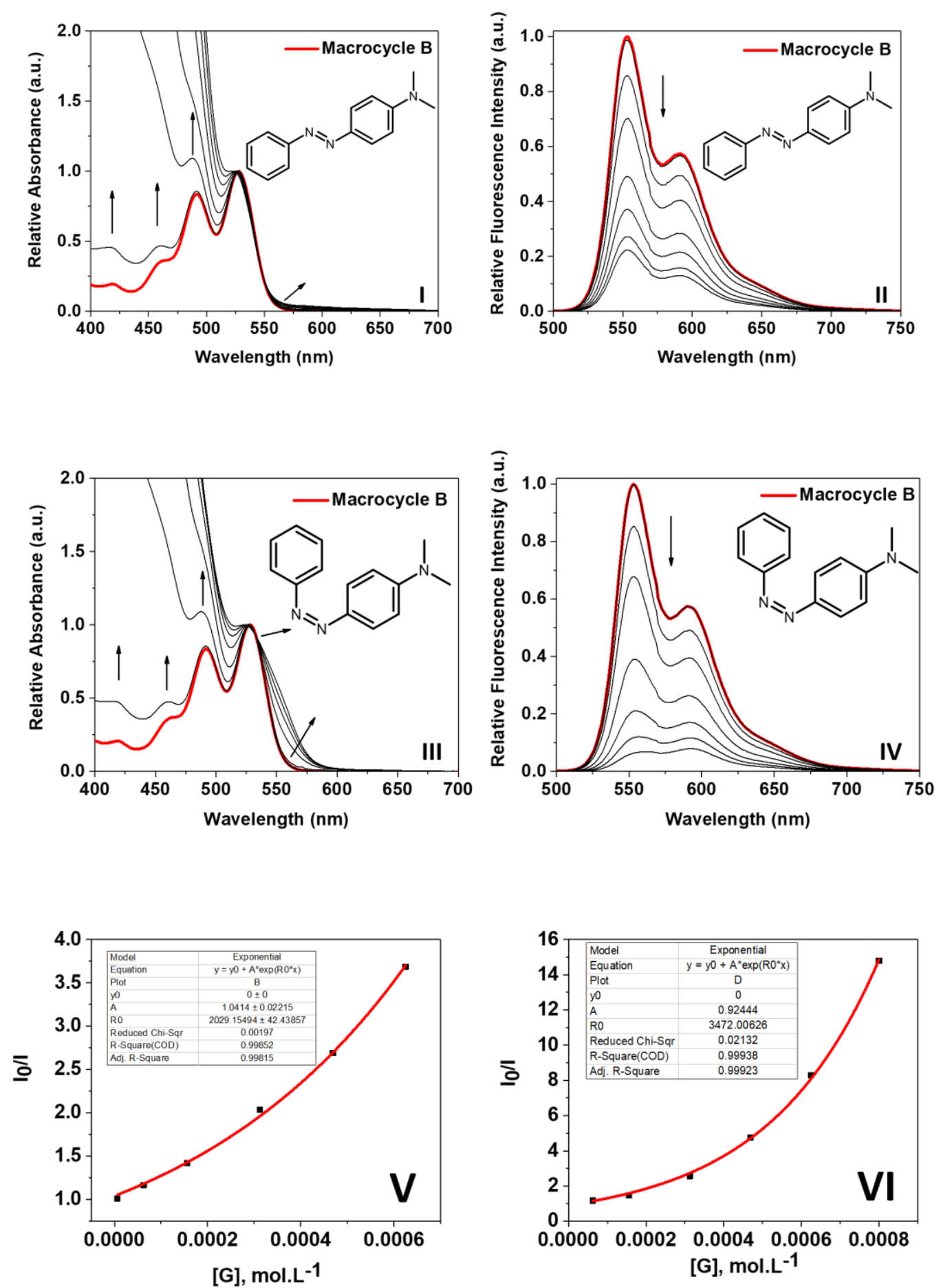

Figure S34. Titration of macrocycle B with $E$ - and Z- dimethylaminoazobenzene in $\mathrm{CHCl}_{3}$. Absorption (I, III) and emission (II, IV) spectra of macrocycle B with $E$ - and Z- 4dimethylaminoazobenzene. Stern-Volmer graphs of fluorescence titrations of macrocycle $B$ with $E$ - 4-dimethylaminoazobenzene (V), Z- 4-dimethylaminoazobenzene (VI). 

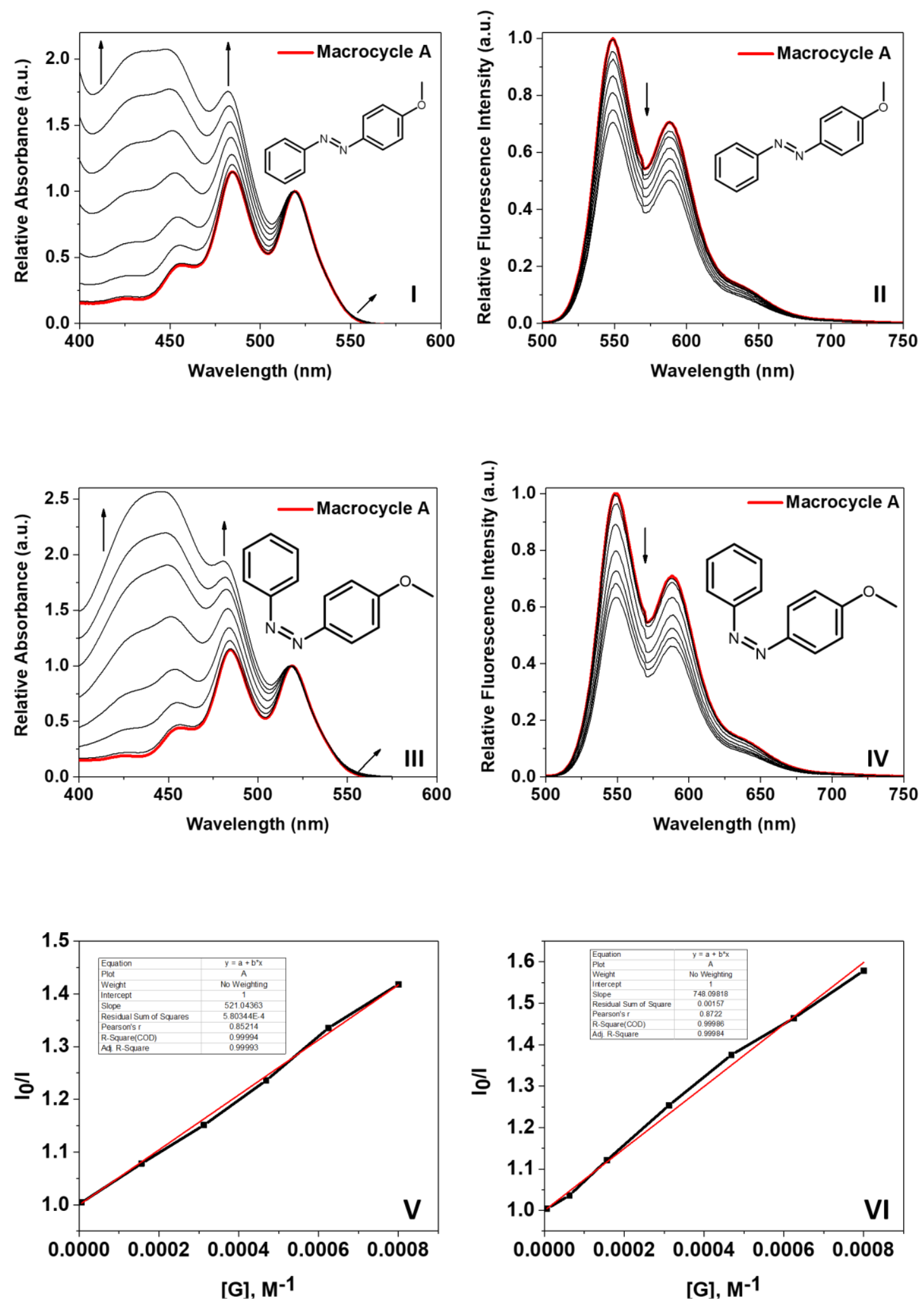

Figure S35. Titration of macrocycle $A$ with $E$ - and Z- 4-methoxyazobenzene in $\mathrm{CHCl}_{3}$. Absorption (I, III) and emission (II, IV) spectra of macrocycle A with $E$ - and Z- 4methoxyazobenzene. Stern-Volmer graphs from emission data of macrocycle A with $E$ - 4methoxyazobenzene (V), Z-4-methoxyazobenzene (VI). 

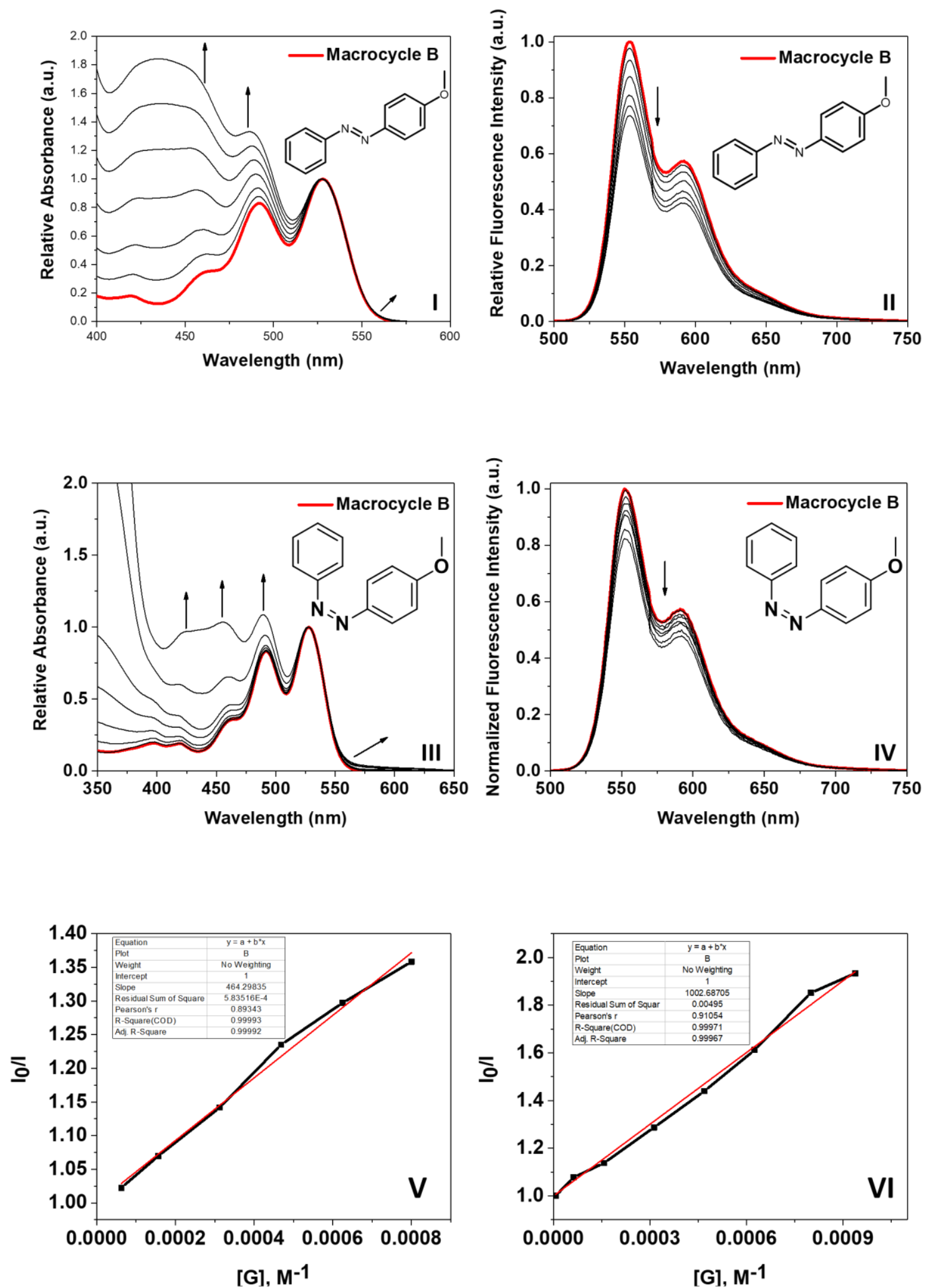

Figure S36. Titration of macrocycle $\mathrm{B}$ with $E$ - and $Z$ - 4-methoxyazobenzene in $\mathrm{CHCl}_{3}$. Absorption (I, III) and emission (II, IV) spectra of macrocycle B with $E$ - and Z- 4methoxyazobenzenes. Stern-Volmer graph from the emission data of macrocycle B with $E$ - 4methoxyazobenzene (V), Z- 4-methoxyazobenzene (VI). 
1. 6. 3D Optimized Structures and Frontier Energy Levels of the Titrants and Stabilized Complexes Obtained by DFT at B3LYP/6$31 G(d)$ Level

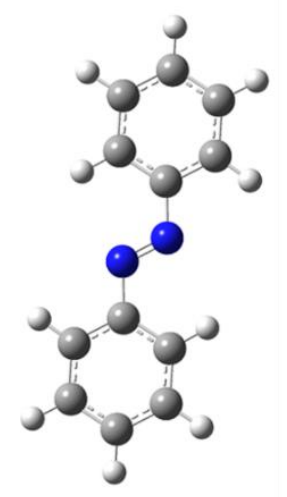

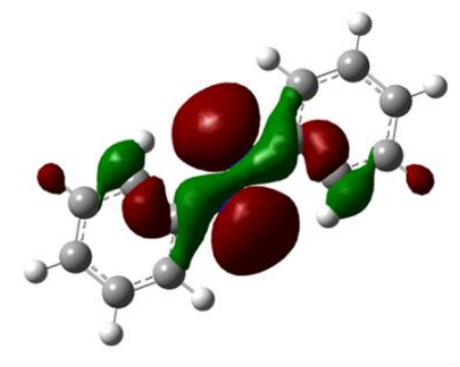

HOMO: -5.97 eV

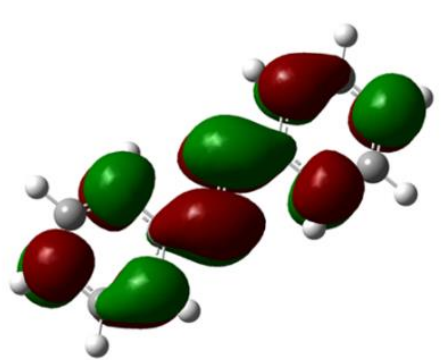

LUMO: $-2.26 \mathrm{eV}$

Figure S37. 3D Structures of the lowest energy conformers and frontier orbitals of EAzobenzene Obtained by DFT at B3LYP/6-31G(d) Level

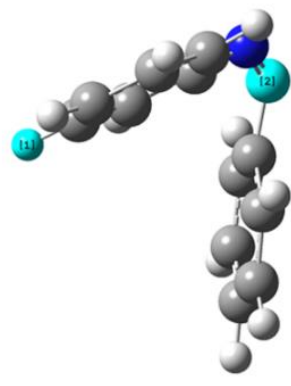

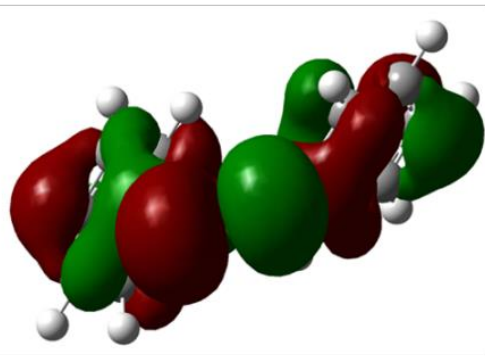

HOMO: -5.63 eV

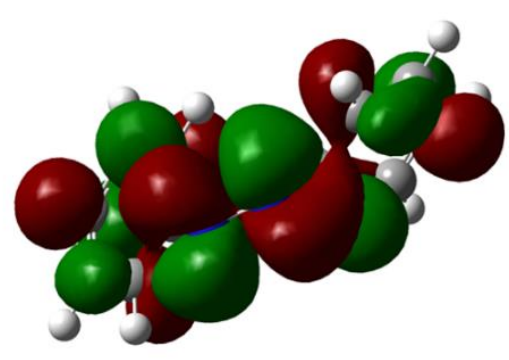

LUMO: $-2.13 \mathrm{eV}$

Figure S38. 3D Structures of the lowest energy conformers and frontier orbitals of Zazobenzene obtained by DFT at B3LYP/6-31G(d) level

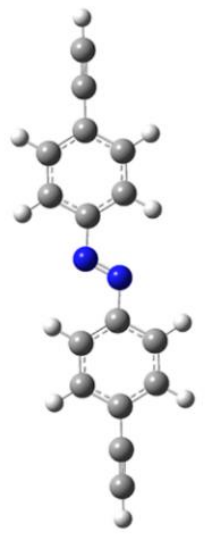

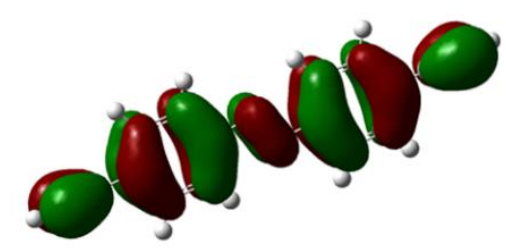

HOMO: $-6.06 \mathrm{eV}$

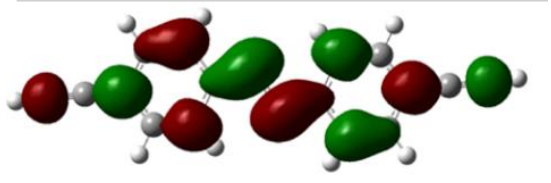

LUMO: -2.64 eV

Figure S39. 3D Structures of the lowest energy conformers and frontier orbitals of E-1,2-bis(4ethynylphenyl)-diazene obtained by DFT at B3LYP/6-31G(d) level 


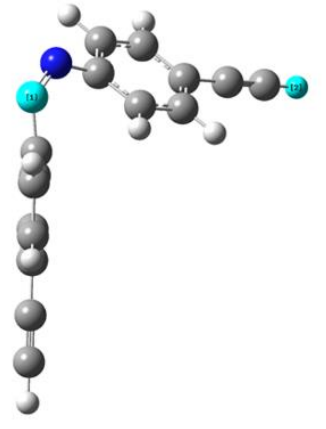

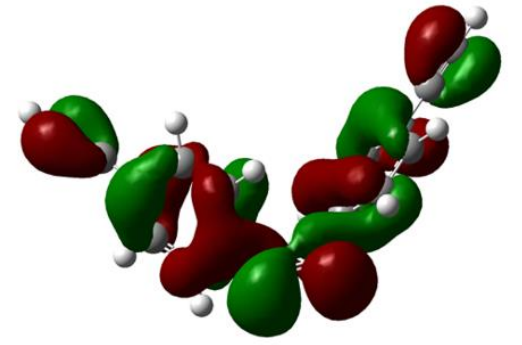

HOMO: $-5.72 \mathrm{eV}$

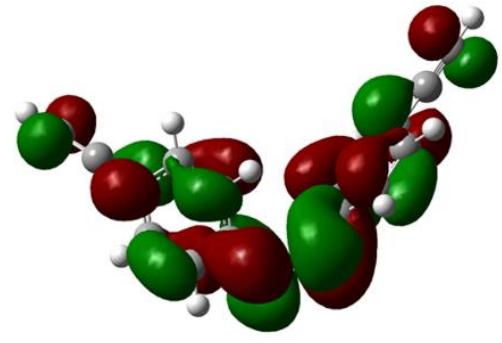

LUMO: -2.51 eV

Figure S40. 3D Structures of the lowest energy conformers and frontier orbitals of Z-1,2-bis(4ethynylphenyl)-diazene obtained by DFT at B3LYP/6-31G(d) level

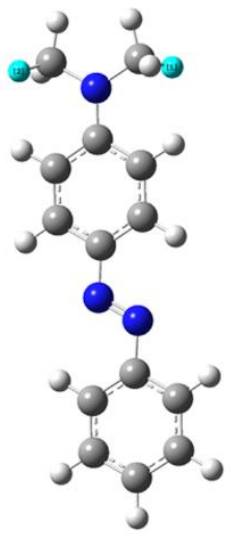

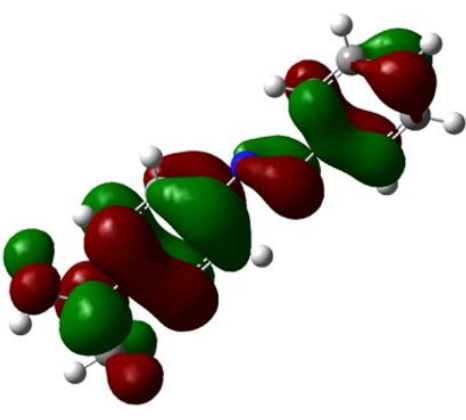

HOMO: -5.10 eV

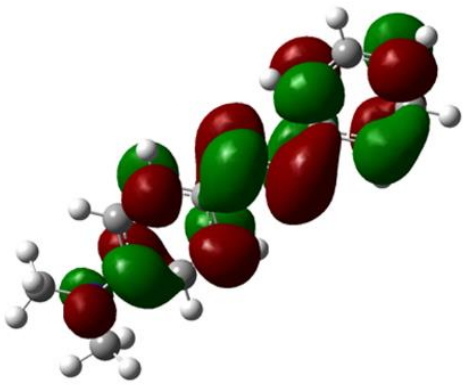

LUMO: -1.79 eV

Figure S41. 3D Structures of the Lowest energy conformers and frontier orbitals of E-4dimethylaminoazobenzene obtained by DFT at B3LYP/6-31G(d) Level

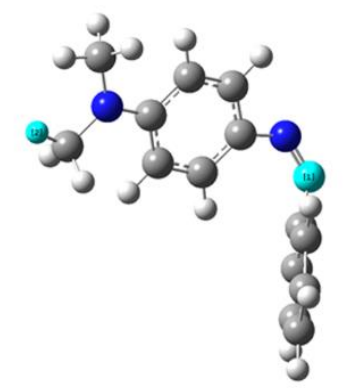

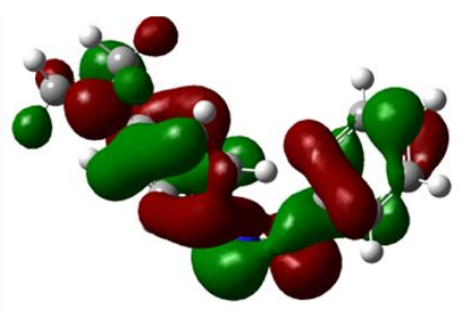

HOMO: -4.99 eV

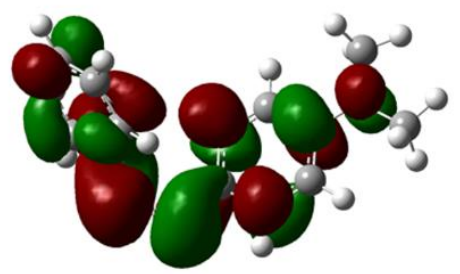

LUMO: -1.68 eV

Figure S42. 3D Structures of the lowest energy conformers and frontier orbitals of Z-4dimethylaminoazobenzene obtained by DFT at B3LYP/6-31G(d) level 


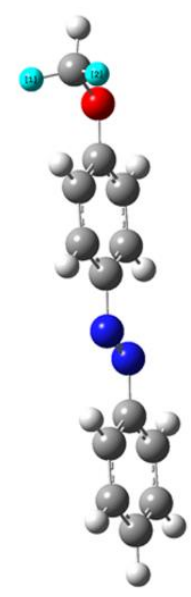

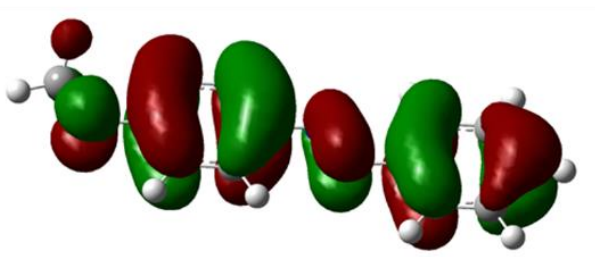

HOMO: $-5.80 \mathrm{eV}$

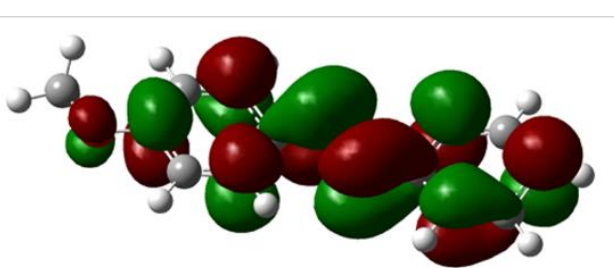

LUMO: -2.09 eV

Figure S43. 3D Structures of the lowest energy conformers and frontier orbitals of E-4methoxyazobenzene obtained by DFT at B3LYP/6-31G(d) level
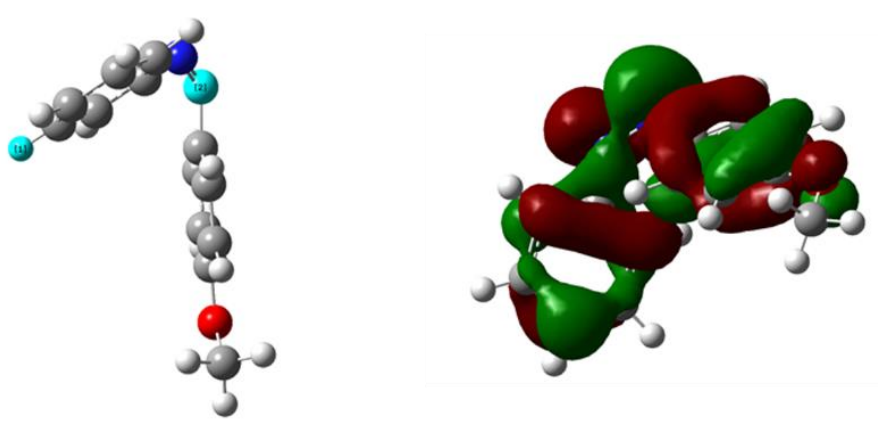

HOMO: $-5.43 \mathrm{eV}$

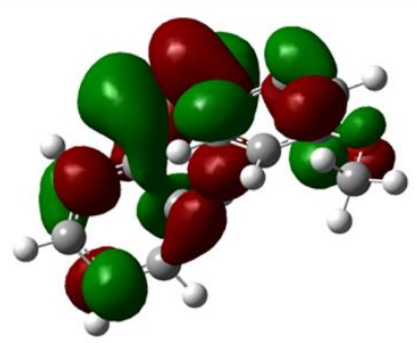

LUMO: $-2.00 \mathrm{eV}$

Figure S44. 3D Structures of the lowest energy conformers and frontier orbitals of Z-4methoxyazobenzene obtained by DFT at B3LYP/6-31G(d) level 


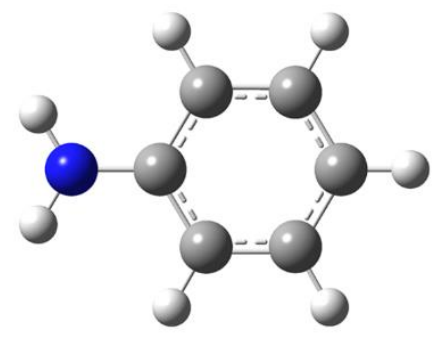

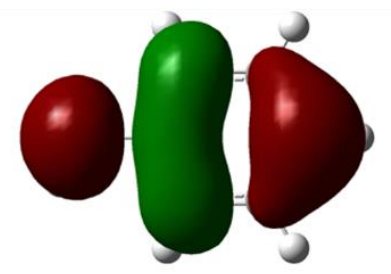

HOMO: -5.15 eV

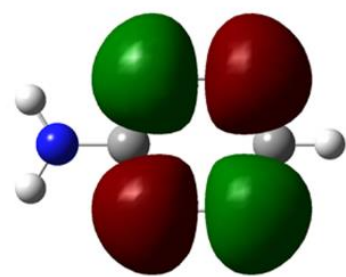

LUMO: 0.37eV

Figure S45. 3D Structures of the lowest energy conformers and frontier orbitals of aniline obtained by DFT at B3LYP/6-31G(d) Level

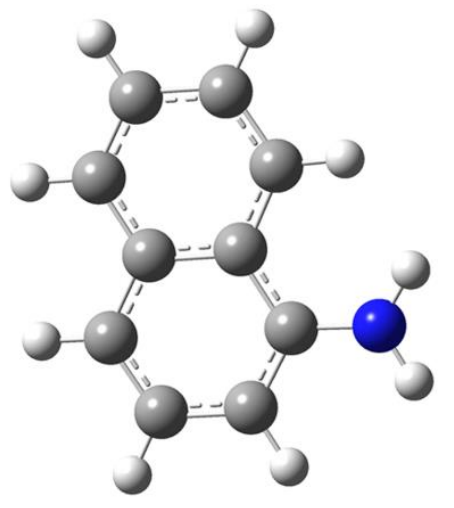

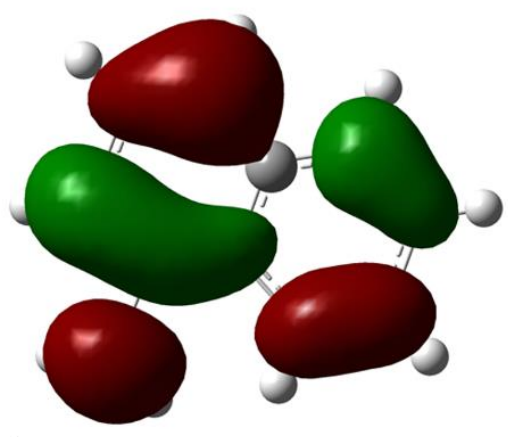

HOMO: $-4.90 \mathrm{eV}$

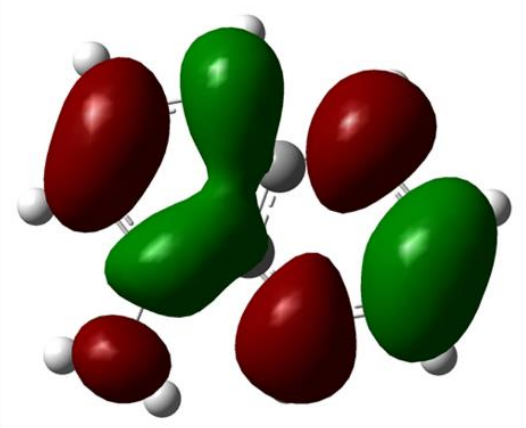

LUMO: $-0.60 \mathrm{eV}$

Figure S46. 3D Structure of the lowest energy conformers and frontier orbitals of 1aminonaphthalene obtained by DFT at B3LYP/6-31G(d) Level

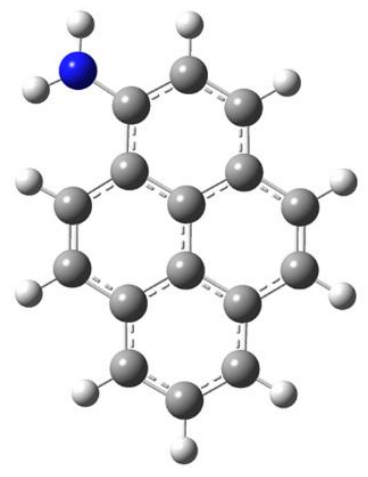

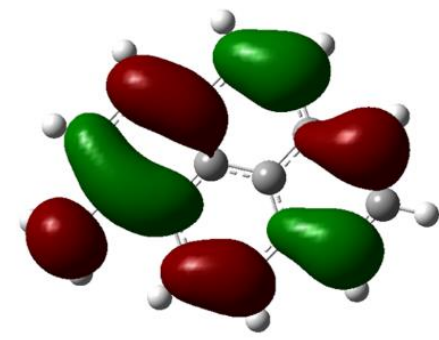

HOMO: $-4.68 \mathrm{eV}$

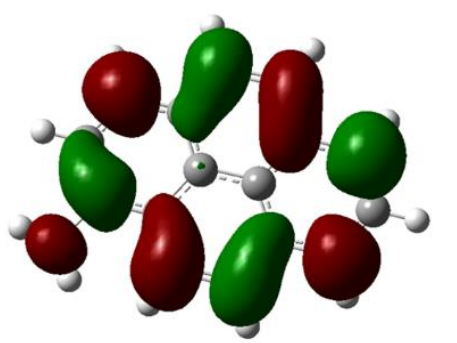

LUMO: -1.14 eV

Figure S47. 3D Structure of the lowest energy conformers and frontier orbitals of 1aminopyrene obtained by DFT at B3LYP/6-31G(d) Level 

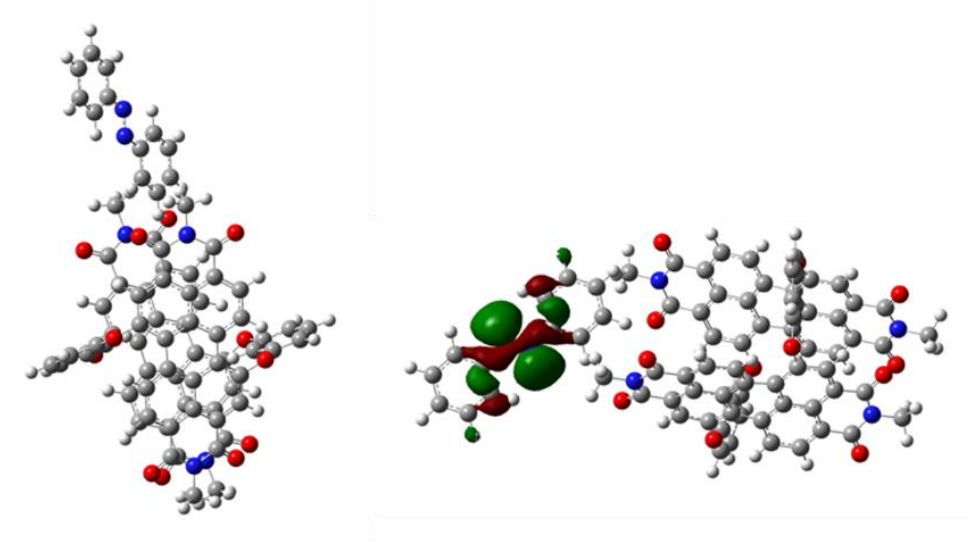

HOMO $=-5.91 \mathrm{eV}$

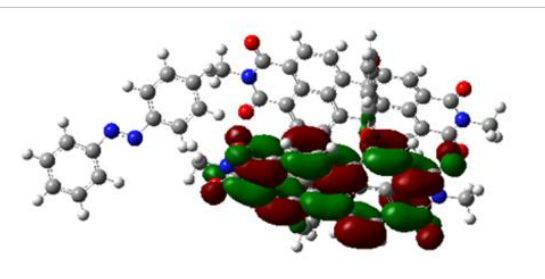

LUMO $=-3.76 \mathrm{eV}$

\section{Band Gap $=2.15 \mathrm{eV}$}

Figure S48. 3D Structures of the lowest energy conformers and frontier orbitals of macrocycle $B \subset E$-azobenzene obtained by DFT at B3LYP/6-31G(d) Level.

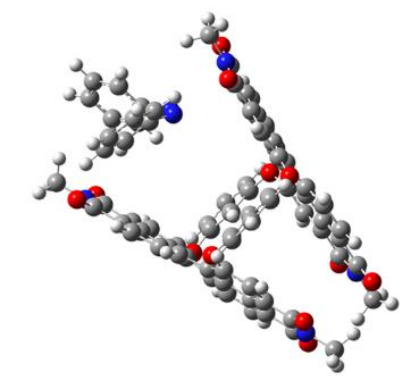

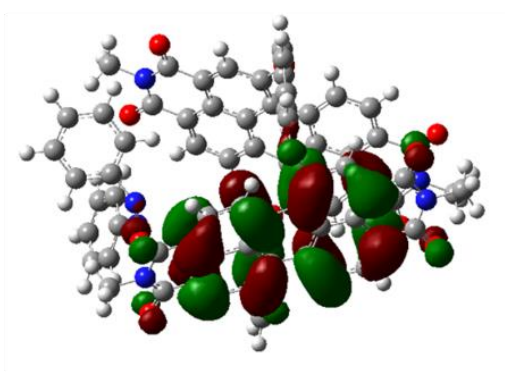

HOMO: -5.75 eV

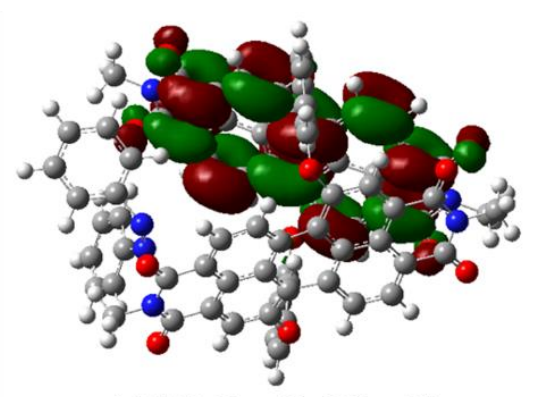

LUMO: -3.66 eV

Band Gap: $2.09 \mathrm{eV}$

Figure S49. 3D Structures of the lowest energy conformers and frontier orbitals of macrocycle B $\subset Z$-azobenzene obtained by DFT at B3LYP/6-31G(d) Level. 


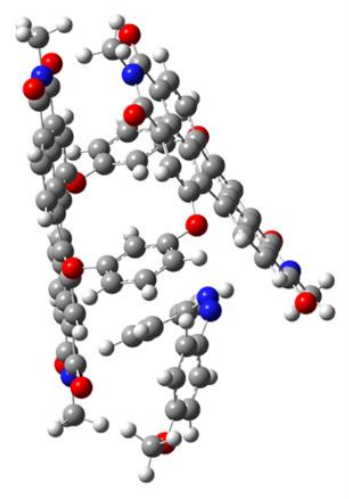

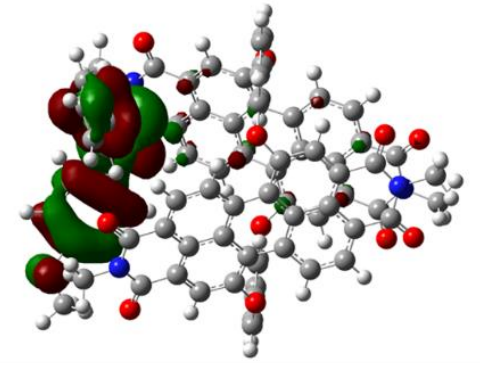

HOMO: $-5.52 \mathrm{eV}$

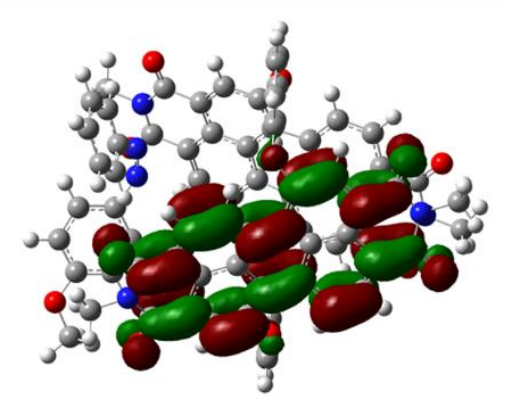

LUMO: $-3.70 \mathrm{eV}$

\section{Band Gap: 1.82 eV}

Figure S50. 3D Structures of the lowest energy conformers and frontier orbitals of macrocycle B $\subset$ Z-4-methoxyazobenzene obtained by DFT at B3LYP/6-31G(d).
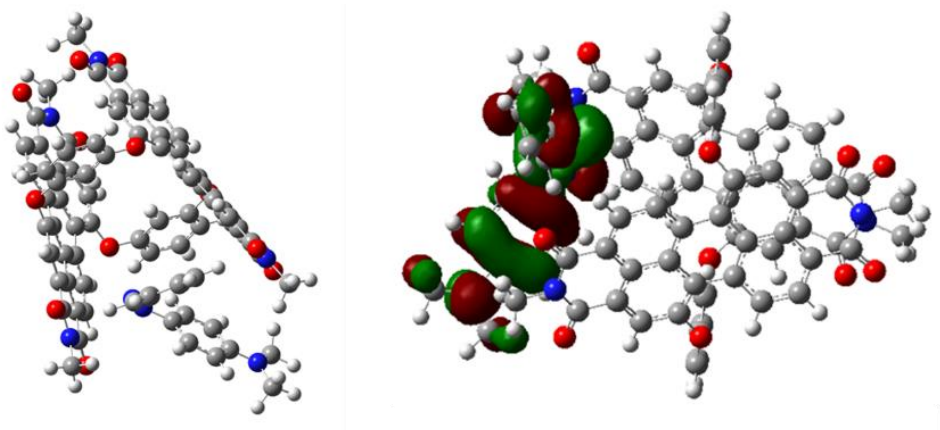

HOMO: -5.06 eV

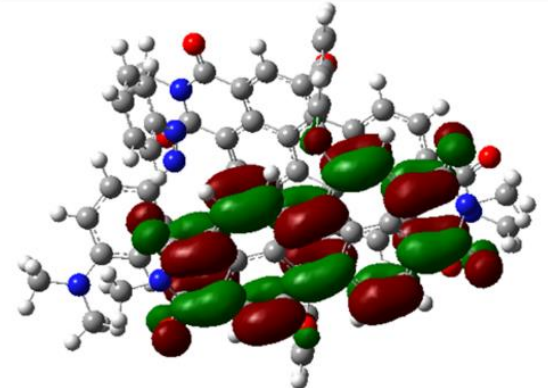

LUMO: $-3.63 \mathrm{eV}$

Band Gap: $1.43 \mathrm{eV}$

Figure S51. 3D Structures of the lowest energy conformers and frontier orbitals of macrocycle B $\subset$ Z-dimethylaminoazobenzene obtained by DFT at B3LYP/6-31G(d). 


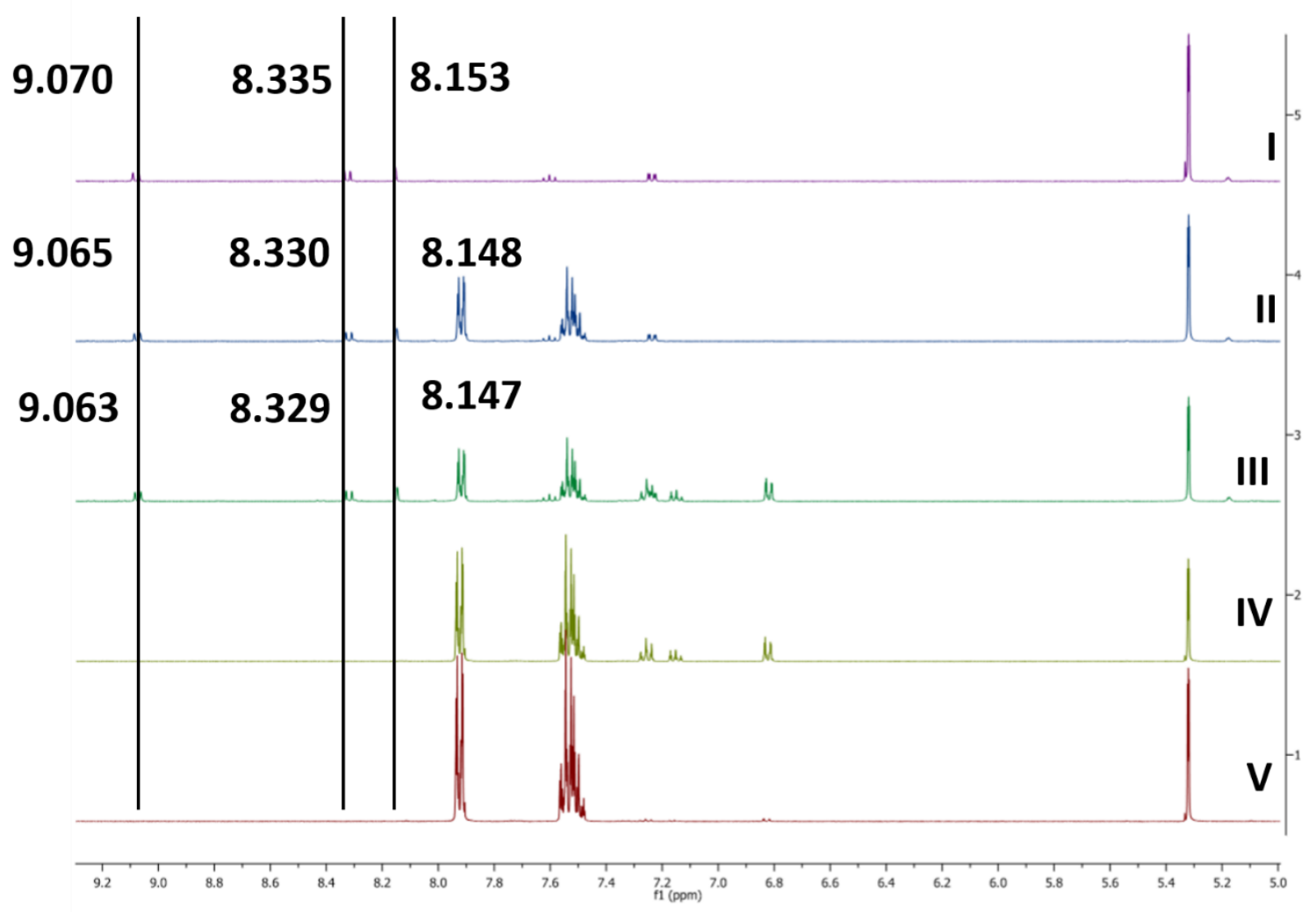

Figure S52. ${ }^{1} \mathrm{H}$ NMR of pure macrocycle $A(I)$, in the presence of 10 equiv. of $E$ - azobenzene (II), upon 30 minutes (III) $1 \mathrm{hr}$ (IV) irradiation at $352 \mathrm{~nm}$ UV light on $E$-azobenzene solution, pure $E$-azobenzene $(\mathrm{V})$ in $\mathrm{CD}_{2} \mathrm{Cl}_{2}$. 


\section{8. ${ }^{1} H-{ }^{1} H$ NOESY NMR of macrocycle $A$ in the presence of E-4- dimethylaminoazobenzene}
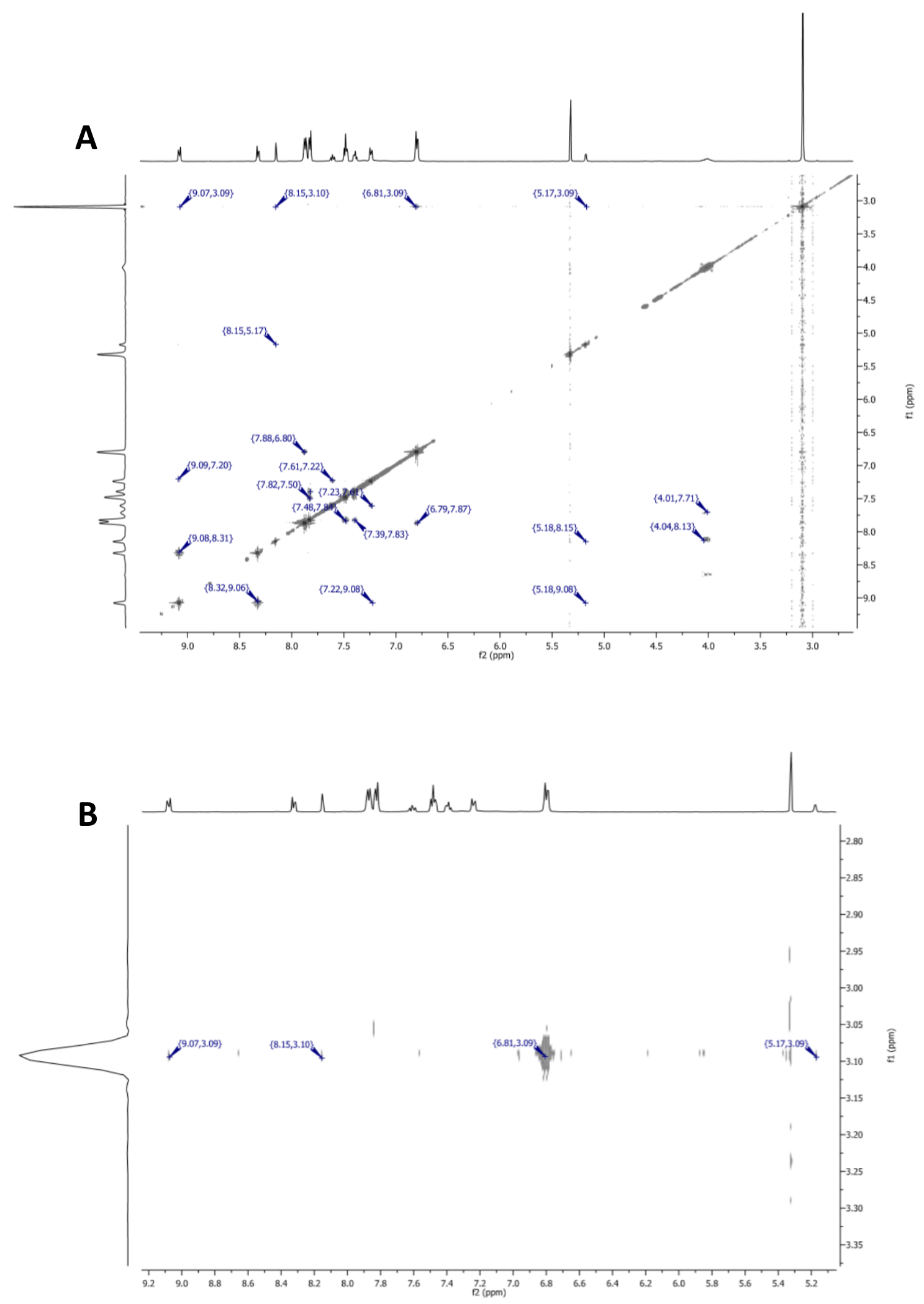

Figure S53. ${ }^{1} \mathrm{H}-{ }^{1} \mathrm{H}$ NOESY NMR macrocycle A with E-4-dimetyhylaminoazobenzene in $\mathrm{CD}_{2} \mathrm{Cl}_{2}$ (A), magnified to show NOE correlations of $\mathrm{N}-\mathrm{CH}_{3}$ peaks (B). 


$$
r_{i j}=r_{r e f} x\left(\frac{a_{r e f}}{a i j}\right)^{1 / 6}
$$

Figure S54. Estimation of the distance $r_{i j}$ between hydrogens $i$ and $j, r_{r e f}=$ distance and $a_{r e f}=$ NOE cross peak volume between known protons, $a_{i j}=$ NOE cross peak volume between protons $\mathrm{i}$ and $\mathrm{j}$

\section{9. Z- azobenzene stabilisation}
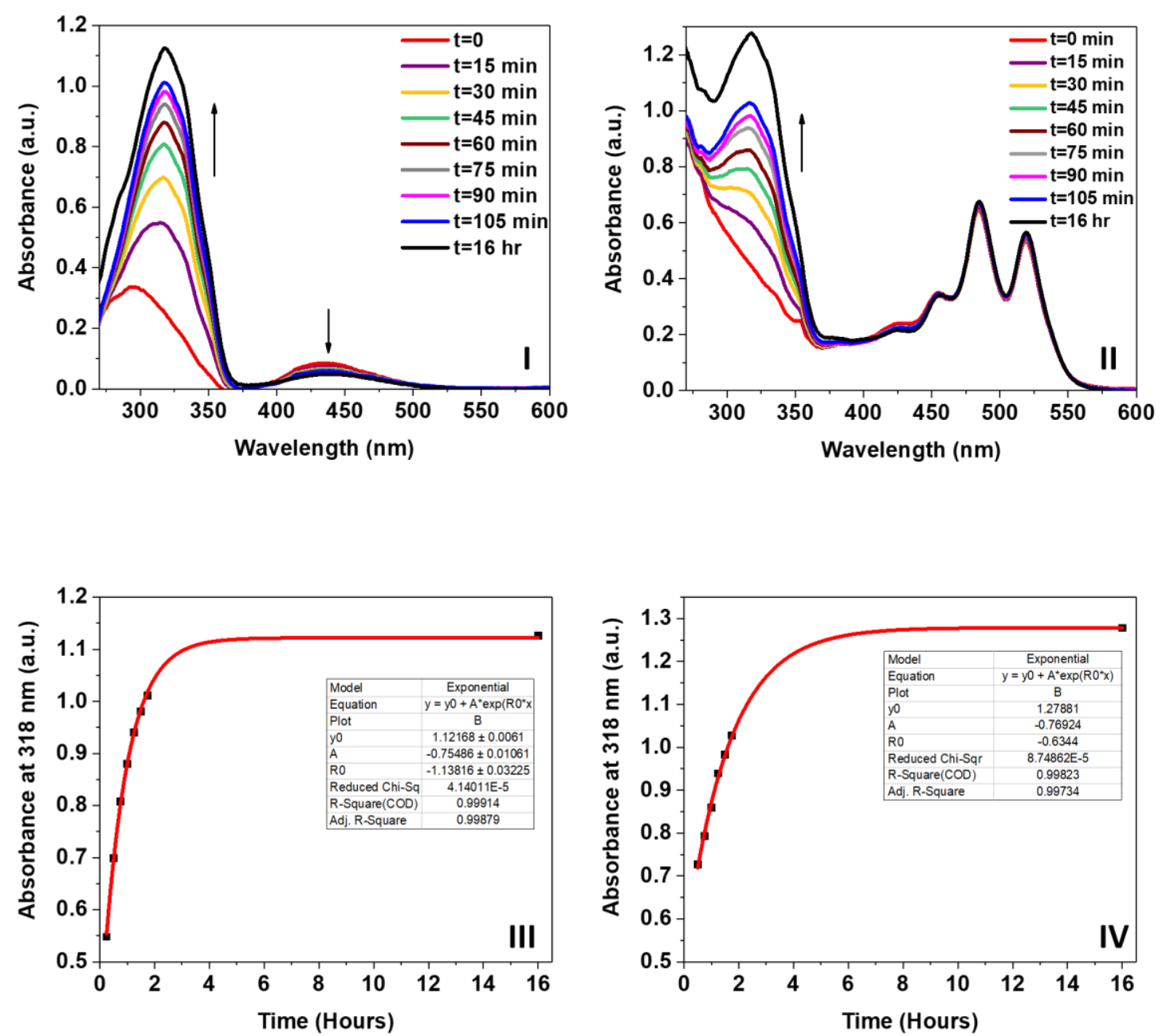

Figure S55. Time dependent absorption spectra of Z-azobenzene (I) and complex of Zazobenzene and macrocycle A (II). Exponential fitting of relaxation of Z-azobenzene (III) and complex of $Z$-azobenzene and macrocycle A(IV) under room light in chloroform. 


\section{10. UV-Vis and fluorescence titrations of macrocycles with}

aromatic amines
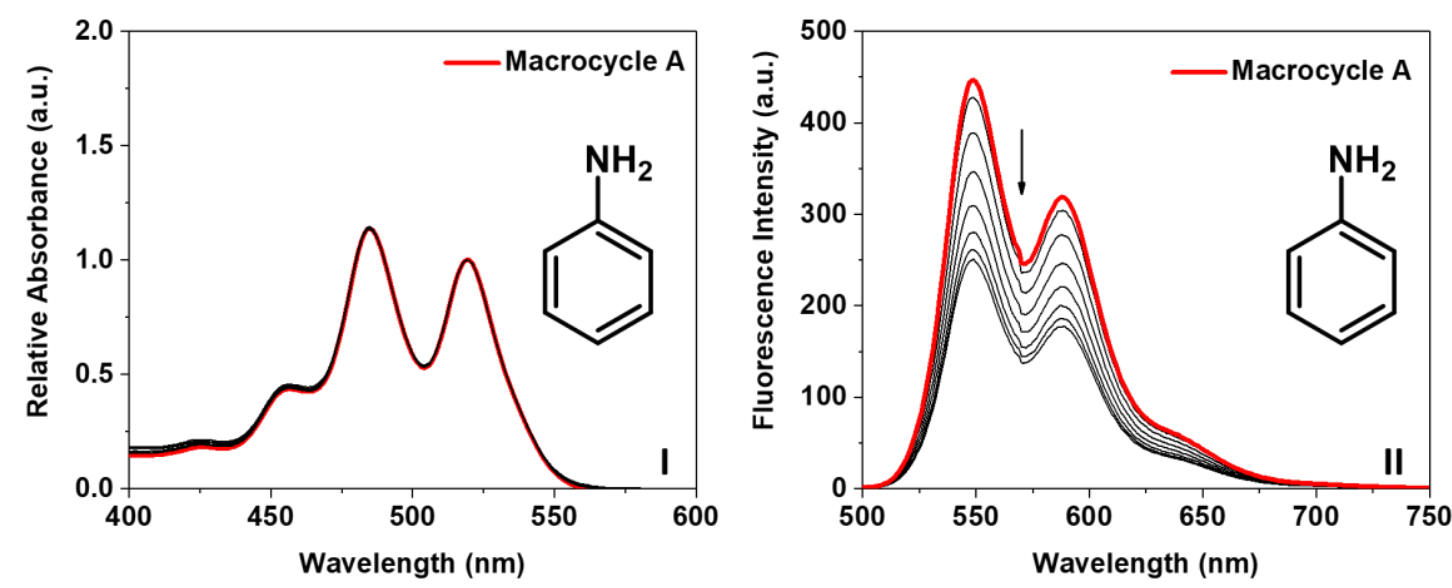

Figure S56. Absorption (I) and emission (II) spectra of macrocycle A in presence of different amounts of aniline in $\mathrm{CHCl}_{3}$
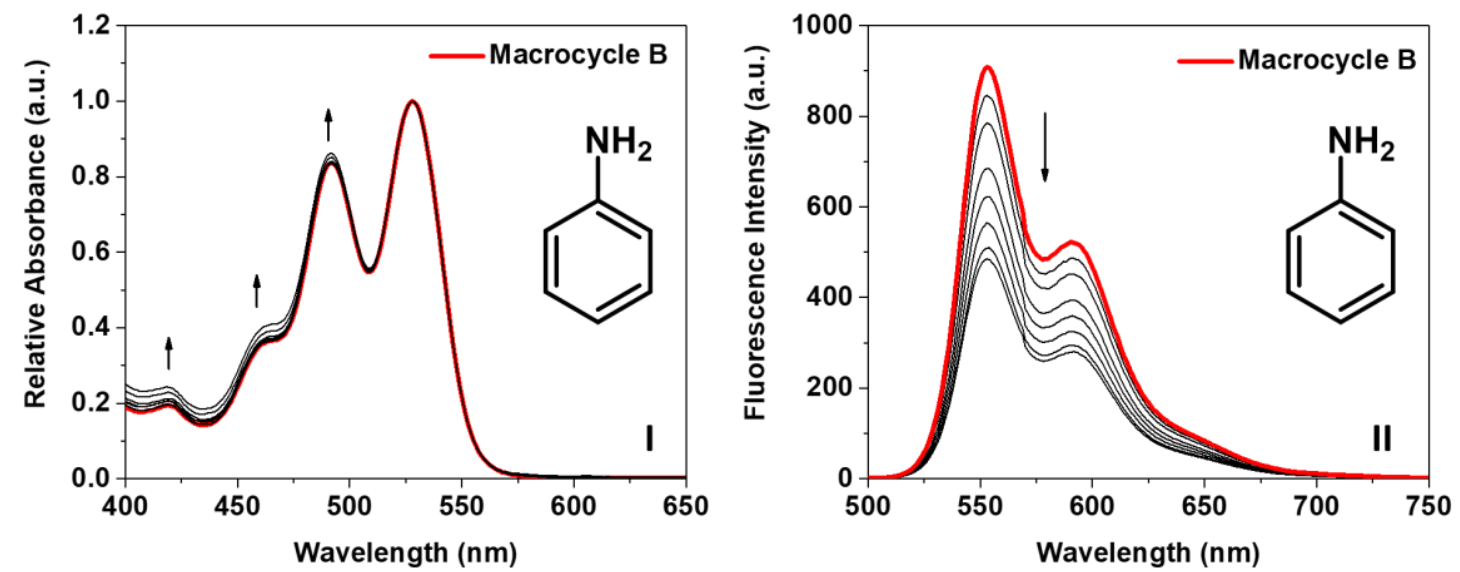

Figure S57. Absorption (I) and emission (II) spectra of macrocycle B in presence of different amounts of aniline in $\mathrm{CHCl}_{3}$ 

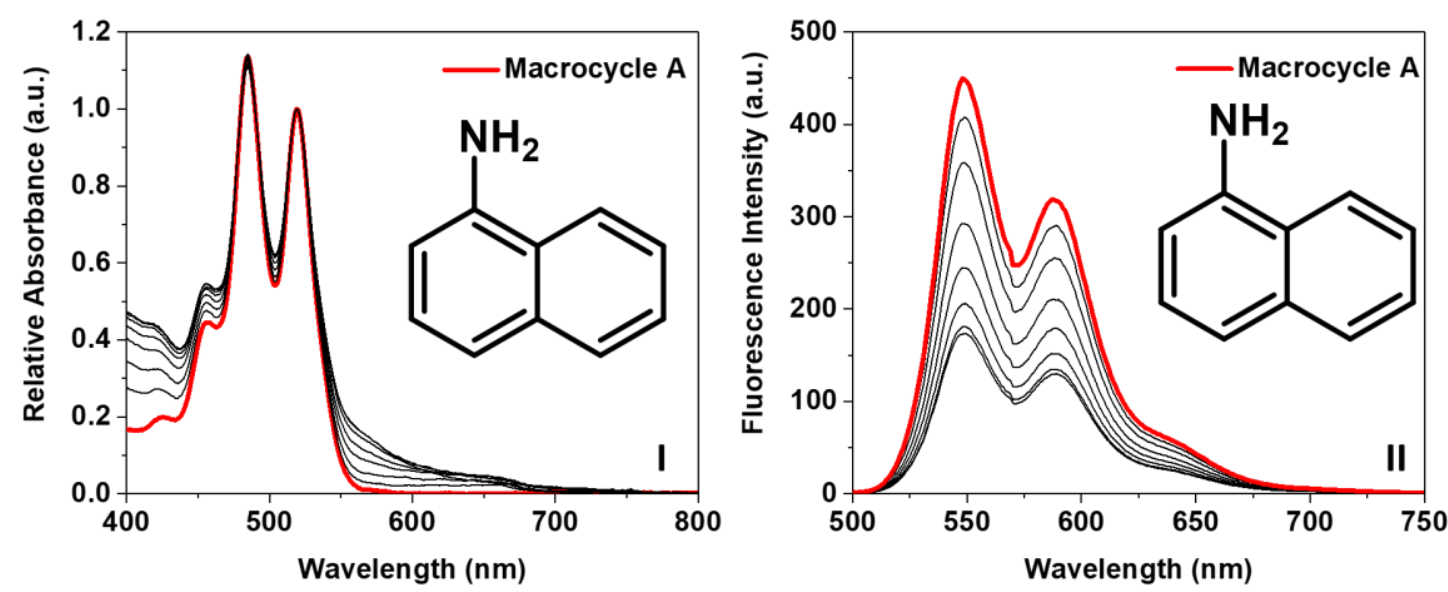

Figure S58. Absorption (I) and emission (II) spectra of macrocycle A in presence of different amounts of 1-aminonaphthalene in $\mathrm{CHCl}_{3}$
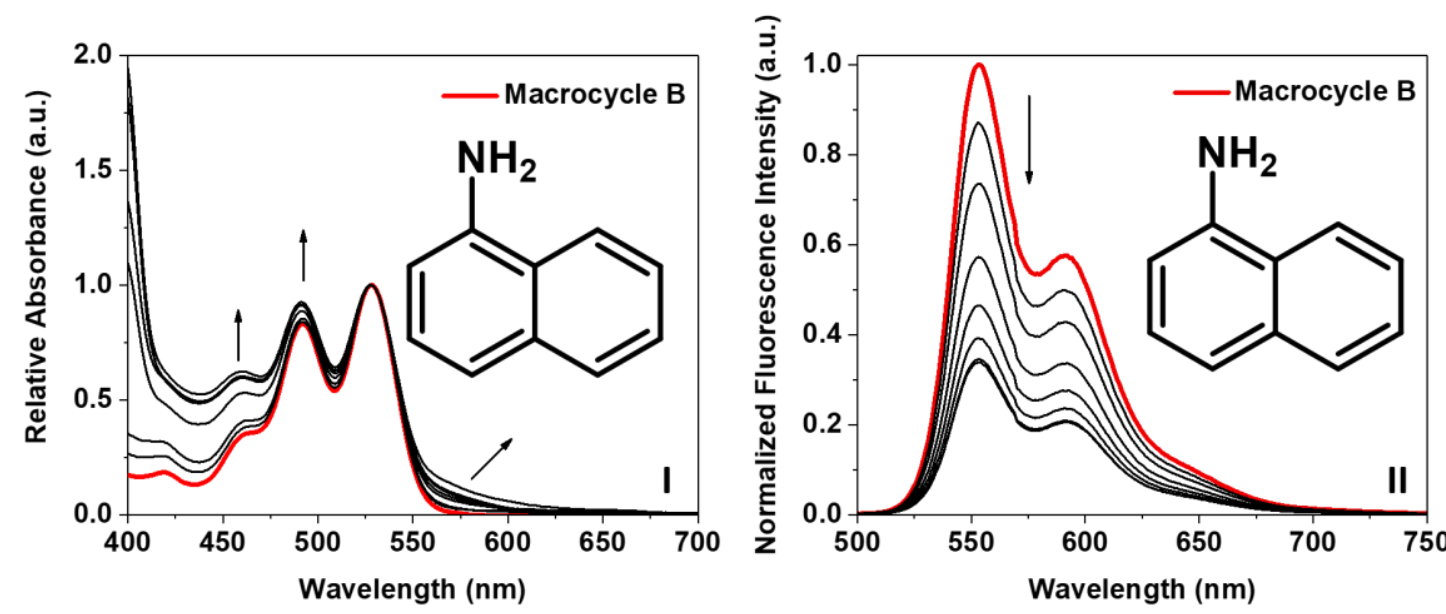

Figure S59. Absorption (I) and emission (II) spectra of macrocycle B in presence of different amounts of 1-aminonaphthalene in $\mathrm{CHCl}_{3}$ 

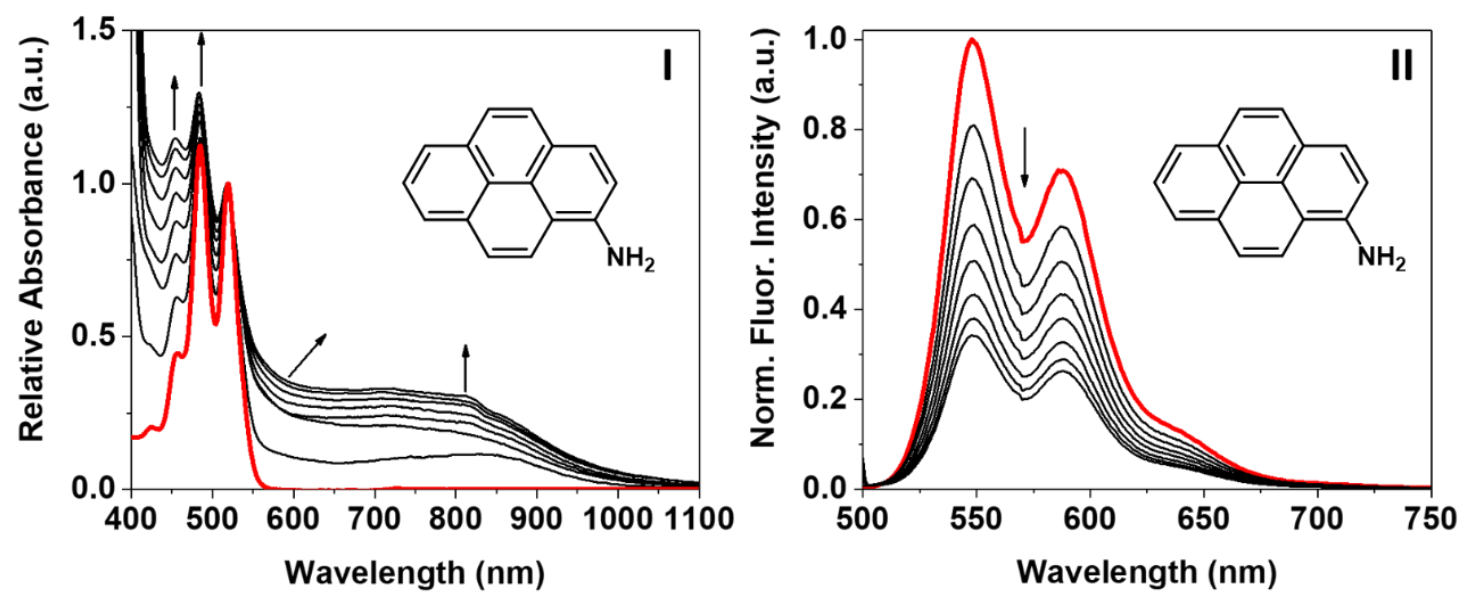

Figure S60. Absorption (I) and emission (II) spectra of macrocycle A in presence of different amounts of 1-aminopyrene in $\mathrm{CHCl}_{3}$
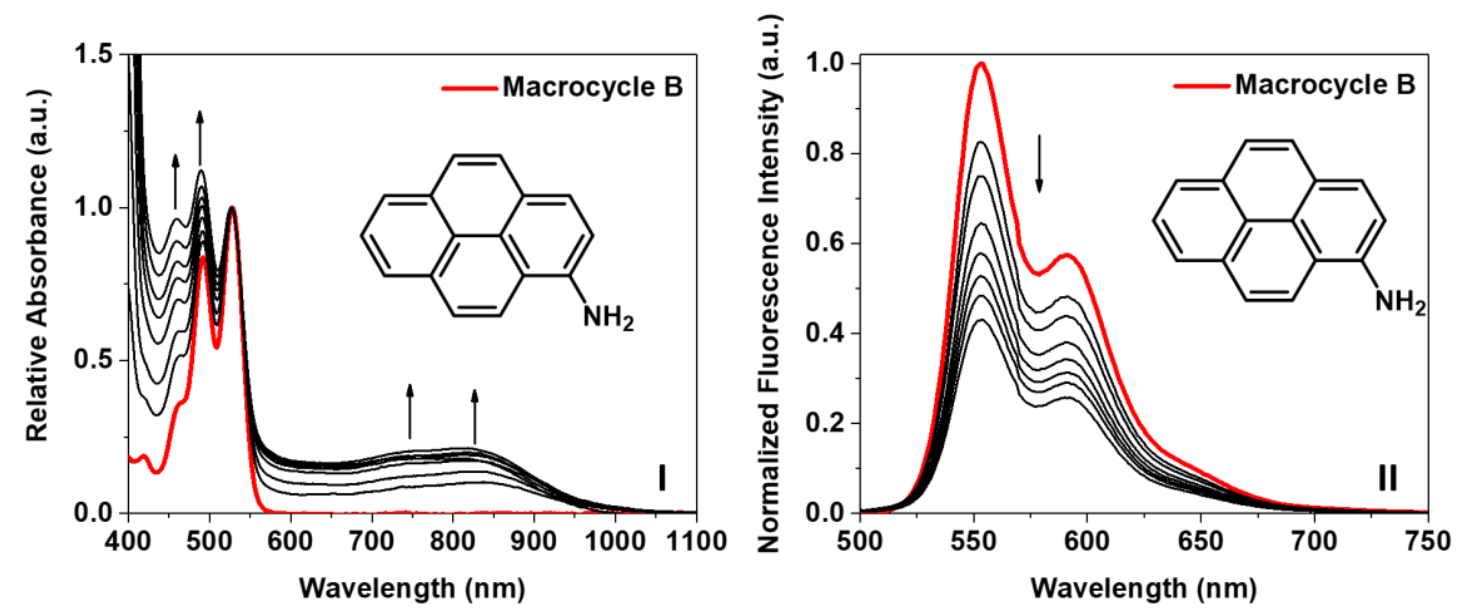

Figure S61. Absorption (I) and emission (II) spectra of macrocycle B in presence of different amounts of 1-aminopyrene in $\mathrm{CHCl}_{3}$ 


\section{11. Self-assembly}
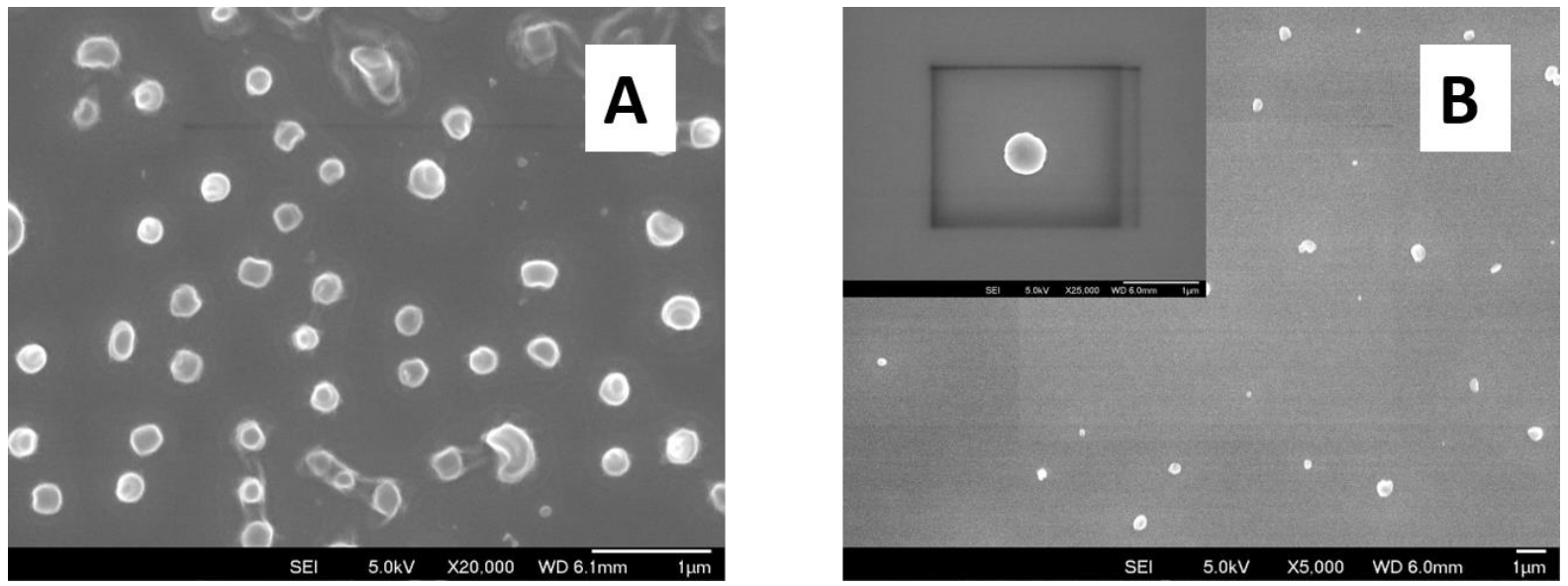

Figure S62. SEM micrographs of particles prepared by drop casting of macrocycle $A(A)$ and macrocycle $B(B)$.
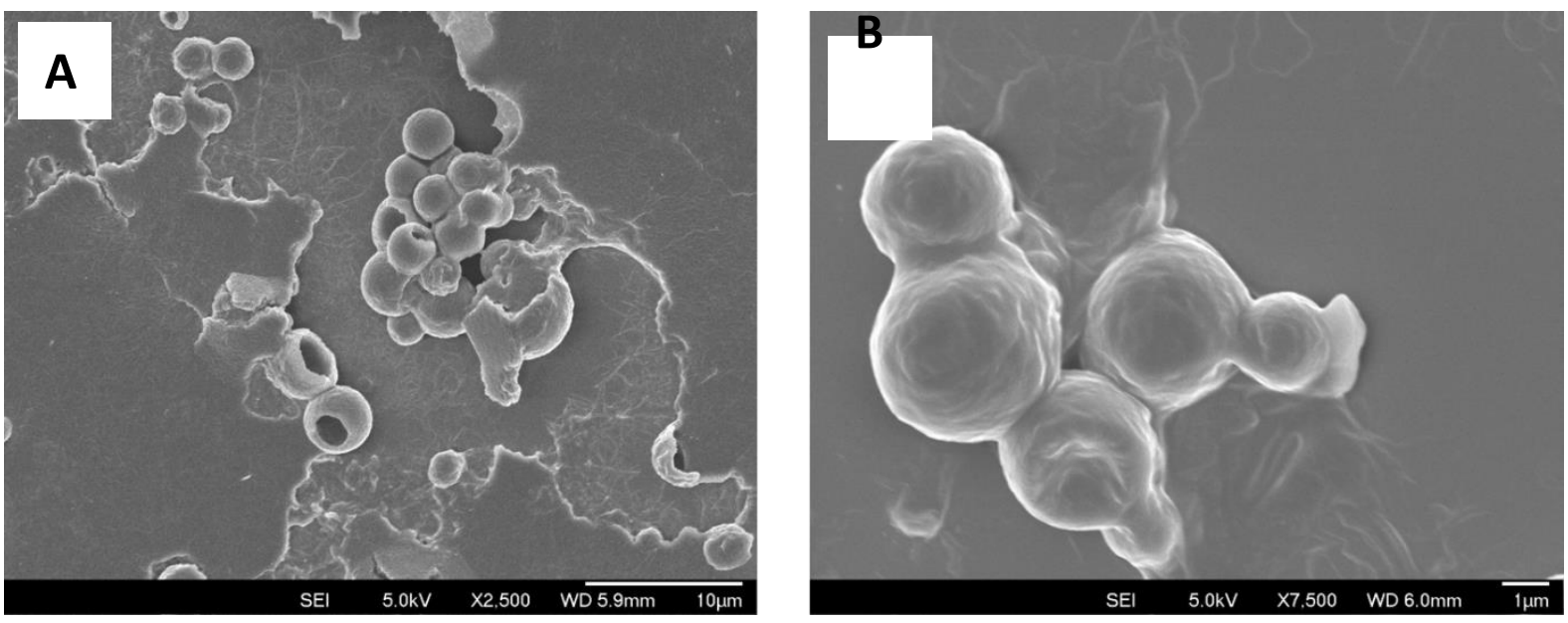

Figure S63. SEM micrographs of drop casted films from an equimolar mixture of macrocycle $B$ and 1,2-bis(4-ethynylphenyl)-diazene (A) and magnified image (B). 